\title{
Recent progress in 2D group-VA semiconductors: from theory to experiment
}

DOI:

$10.1039 / \mathrm{c} 7 \mathrm{cs} 00125 \mathrm{~h}$

\section{Document Version}

Accepted author manuscript

Link to publication record in Manchester Research Explorer

\section{Citation for published version (APA):}

Zhang, S., Guo, S., Chen, Z., Wang, Y., Gao, H., Gomez-Herrero, J., Ares, P., Zamora, F., Zhu, Z., \& Zeng, H. (2018). Recent progress in 2D group-VA semiconductors: from theory to experiment. Chemical Society Reviews, 47(3), 982-1021. https://doi.org/10.1039/c7cs00125h

\section{Published in:}

Chemical Society Reviews

\section{Citing this paper}

Please note that where the full-text provided on Manchester Research Explorer is the Author Accepted Manuscript or Proof version this may differ from the final Published version. If citing, it is advised that you check and use the publisher's definitive version.

\section{General rights}

Copyright and moral rights for the publications made accessible in the Research Explorer are retained by the authors and/or other copyright owners and it is a condition of accessing publications that users recognise and abide by the legal requirements associated with these rights.

\section{Takedown policy}

If you believe that this document breaches copyright please refer to the University of Manchester's Takedown Procedures [http://man.ac.uk/04Y6Bo] or contact uml.scholarlycommunications@manchester.ac.uk providing relevant details, so we can investigate your claim.

\section{OPEN ACCESS}




\title{
Recent progress in 2D group-VA semiconductors: from theory to experiment
}

\author{
Shengli Zhang, ${ }^{\mathrm{a}}$ Shiying Guo, ${ }^{\mathrm{a}}$ Zhongfang Chen, ${ }^{\mathrm{b}}$ Yeliang Wang, ${ }^{\mathrm{c}}$ Hongjun \\ Gao, ${ }^{\mathrm{c}}$ Julio Gómez-Herrero, ${ }^{\mathrm{d}}$ Pablo Ares, ${ }^{\mathrm{d}}$ Félix Zamora, ${ }^{\mathrm{e}}$ Zhen $\mathrm{Zhu}{ }^{\mathrm{f}}$ and \\ Haibo Zeng* $*^{\mathrm{a}}$
}

${ }^{a}$ Institute of Optoelectronics \& Nanomaterials, Herbert Gleiter Institute of Nanoscience College of

Materials Science and Engineering, Nanjing University of Science and Technology, Nanjing, 210094,

China

${ }^{b}$ Department of Chemistry, Institute for Functional Nanomaterials, University of Puerto Rico, Rio

Piedras, San Juan, PR 00931, USA

${ }^{c}$ Institute of Physics and University of Chinese Academy of Sciences Chinese Academy of Sciences

Beijing 100190, China

${ }^{\mathrm{d}}$ Departamento de Física de la Materia Condensada, Universidad Autónoma de Madrid Madrid E

28049, Spain

e Departamento de Química Inorgánica, Universidad Autónoma de Madrid Madrid E 28049, Spain

${ }^{\mathrm{f}}$ Materials Department, University of California, Santa Barbara, California 93106, USA

EMAIL: felix.zamora@uam.es, zeng.haibo@njust.edu.cn

Phosphorene, an emerging two-dimensional material, has received considerable attention due to its layercontrolled direct bandgap, high carrier mobility, negative Poisson's ratio and unique in-plane anisotropy. As cousins of phosphorene, 2D group-VA arsenene, antimonene and bismuthene have also garnered tremendous interest due to their intriguing structures and fascinating electronic properties. 2D group-VA family members are opening up brand-new opportunities for their multifunctional applications encompassing electronics, optoelectronics, topological spintronics, thermoelectrics, sensors, Li- or Nabatteries. In this review, we extensively explore the latest theoretical and experimental progress made in the fundamental properties, fabrications and applications of $2 \mathrm{D}$ group-VA materials, and offer perspectives and challenges for the future of this emerging field.

\section{Introduction}

Two-dimensional (2D) materials possess rather unique and exceptional properties, which are of great importance for new-generation smart electronic, optoelectronic and energy devices due to their finite band gap, superior flexibility, absence of dangling bonds and the significant resistance to short channel effects. $^{1-13}$ A family of 2D crystals, derived from group-VA layered materials (P, As, Sb, Bi), has emerged with increasing research interest, strong momentum of development and promising practical applications. $^{14-69}$ Different from semimetallic group-IVA (graphene, silicene, germanene and stanene) 
and metallic group-IIIA (borophene) materials, ${ }^{70-78} 2 \mathrm{D}$ nanosheets of the group-VA elements are semiconductors with significant fundamental band gaps, thus rendering them potential candidates for future nanodevices.

Among 2D group-VA family members, the first and also the most explored one is phosphorene, which exhibits a direct band gap with large tunability, from $0.3 \mathrm{eV}$ in the bulk to $2.0 \mathrm{eV}$ in monolayers, and a high hole mobility above $10000 \mathrm{~cm}^{2} \mathrm{~V}^{-1} \mathrm{~s}^{-1} \cdot{ }^{14-17}$ In 2014 , Li et al. successfully fabricated the first phosphorene field-effect transistor (FET) based on micrometer-sized flakes of few-layer black phosphorus. ${ }^{16}$ Besides, monolayer and few-layer phosphorene also present other promising properties, such as highly anisotropic transport, superior mechanical properties, negative Poisson's ratios, excellent optical and thermoelectric responses, strain-induced conduction bands, and perpendicular electric-fieldinduced 2D topological character. ${ }^{17-25,79}$ These unusual properties inspire a recent flourish of studies on phosphorene, which holds great potential for future applications in many fields.

Over the past two years, as the cousins of phosphorene, group-VA arsenene, antimonene and bismuthene have also attracted lots of interest from physicists, chemists and material scientists. ${ }^{26-69}$ Particularly, in 2015, Zhang et al. predicted and identified novel 2D semiconducting As and Sb monolayers, namely arsenene and antimonene with wide band gaps, desirable stability and high carrier mobility. ${ }^{26}$ After that, many available theoretical studies further revealed their rich and peculiar properties. Importantly, the theoretically predicted monolayer and few-layer group-VA nanosheets have been fabricated experimentally by mechanical exfoliation, liquid phase exfoliation, plasma-assisted processes, vapor deposition techniques and molecular beam epitaxy. ${ }^{30-34,80-85}$

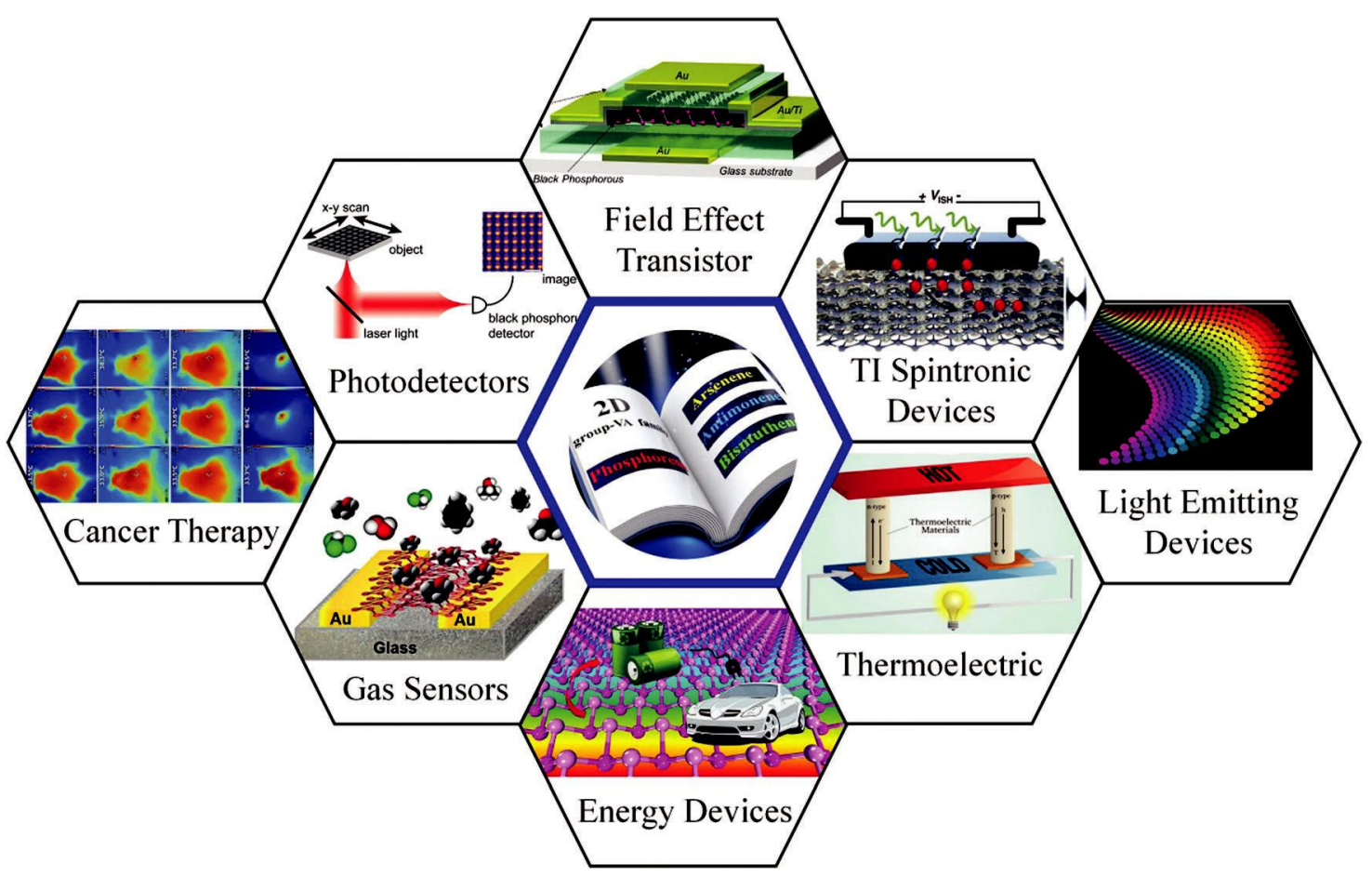

Fig. 1 Applications of 2D group-VA materials in field effect transistors (reproduced from ref. 224 with permission from the American Chemical Society, copyright 2015), photodetectors (reproduced from ref. 318 with permission from the American Chemical Society, copyright 2014), TI spintronic devices ${ }^{93}$ (reproduced from ref. 93 with permission from the American Chemical Society, copyright 2015), cancer therapy (reproduced from ref. 360 with permission from Wiley, copyright 2017), light emitting devices, gas sensors (reproduced from ref. 333 with permission from Wiley, copyright 2015), thermoelectric and energy devices. 
With significant band gaps, predicted theoretically, and desirable high stability, as proved experimentally, 2D group-VA materials are expected to be promising and competitive candidates for electronic, optoelectronic, spintronic and thermoelectric applications (Fig. 1). Further theoretical and experimental explorations of these group-VA materials will not only broaden our fundamental knowledge of the 2D semiconducting family, but also pave a practical route towards various technological applications. With these efforts, it is anticipated that these novel 2D group-VA materials can complement well the properties of graphene and other low dimensional materials to establish a new research branch in the field of nanomaterials. Some groups have reviewed the physical and chemical characteristics of elemental 2D materials. However, they mainly place emphasis on graphene, silicene, germanene, stanene, phosphorene and borophene. $^{71,86-92}$ The rapidly increasing number of publications in the area of groupVA materials provides a strong motivation for this comprehensive review.

Herein, we report a systematic review of the latest advances in 2D group-VA materials (phosphorene, arsenene, antimonene and bismuthene) and discuss their fundamental properties and applications. First, we will highlight the structural properties of this $2 \mathrm{D}$ semiconductor family, as well as their layered bulk counterparts. Then, we will describe the unprecedented electronic properties of 2D group-VA systems in detail. Furthermore, the synthesis approaches and their applications will be broadly discussed. Finally, a perspective on 2D group-VA semiconductors will be provided.

\section{Structures and properties of group-VA 2D semiconductors}

Group-VA layered bulk materials show a structural variety, being their typical allotropes. Significantly, phosphorus has white, red, black phosphorus and other several amorphous forms. ${ }^{94-96}$ Black phosphorus is the most thermodynamically stable allotropic form under ambient conditions. It crystallizes in a layered orthorhombic structure with space group Cmca, consisting of parallel puckered double-floor atomic layers. Within the individual atomic layer, each phosphorus atom has five valence electrons from the $3 \mathrm{~s}$ and $3 \mathrm{p}$ orbitals, which hybridize to form three covalent bonds with neighboring atoms. Among them, two covalent bonds lie parallel with the atomic plane while the third one is nearly perpendicular to that and plays an important role in bridge connection of phosphorus atoms from upper and lower layers, forming a unique puckered structure (a phase) as shown in Fig. 2a. Bulk black phosphorus exhibits a band gap of $0.3 \mathrm{eV}$, a high carrier mobility of $10^{3} \mathrm{~cm}^{2} \mathrm{~V}^{-1} \mathrm{~s}^{-1}$ and a highly anisotropic layered structure. ${ }^{97,98}$ Under approximately $5 \mathrm{GPa}$, black phosphorus transforms into a semimetallic $\mathrm{b}$ phase with space group R3\%m as shown in Fig. 2b. ${ }^{99}$ The b phase is crystallized in a layered rhombohedral structure, which adopts a double-layered structure consisting of many interlocked, ruffled and six-membered rings. For black phosphorus, its first isolation could be traced back to one hundred years ago. The original synthetic procedure is based on the treatment of white phosphorus at $1.2 \mathrm{GPa}$ and $1 \mathrm{C}$ for 30 minutes. ${ }^{100-102}$ In the subsequent years, black phosphorus crystals were usually obtained from white or red phosphorus under high pressure and high temperature conditions. ${ }^{97,103,104}$ Further improvement of crystal growth methods of black phosphorus has been more explored at reduced temperature and pressure. ${ }^{105-107}$

Arsenic has three common allotropes, which are metallic gray, yellow and black arsenic. ${ }^{108}$ Gray arsenic is not only the most common phase but also the most stable form with a layered rhombohedral structure (space group R3\%m), as shown in Fig. 2b. Importantly, the layered gray arsenic crystal also exists in nature and has been known for more than a thousand years. Gray arsenic is a semimetal material with bands near the $\mathrm{T}$ and $\mathrm{L}$ points partly overlapped. ${ }^{109}$ When arsenic is heated to approximately $370 \mathrm{~K}$, a layered orthorhombic a phase arises, which holds a similar black phosphorus structure. ${ }^{110}$ The orthorhombic arsenic is a narrow-gap semiconductor with a band gap of approximately $0.3 \mathrm{eV} .{ }^{111}$ 
(a)

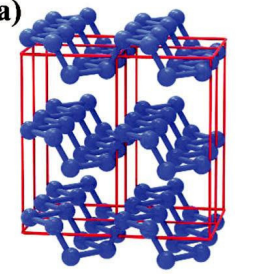

a phase, group Cmca (b)
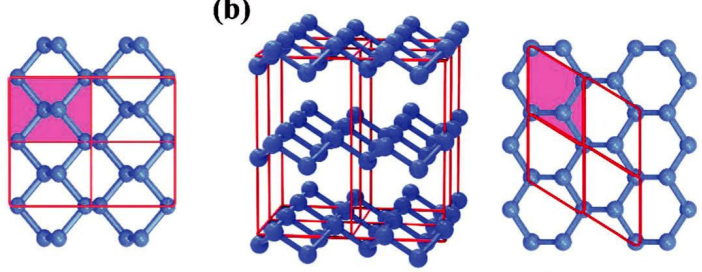

B phase, group $R \overline{3} m$

(c)

\begin{tabular}{|c|c|c|c|}
\hline Elements & \multicolumn{2}{|c|}{$\begin{array}{c}\text { Stable } \\
\text { phase }\end{array}$} & $\begin{array}{c}\text { Natural layered } \\
\text { crystal }\end{array}$ \\
\hline $\boldsymbol{P}$ & $\boldsymbol{\alpha}$ & $\boldsymbol{\beta}$ & $\boldsymbol{/}$ \\
\hline As & $\boldsymbol{\alpha}$ & $\boldsymbol{\beta}$ & $\boldsymbol{\beta}$ \\
\hline Sb & $/$ & $\boldsymbol{\beta}$ & $\boldsymbol{\beta}$ \\
\hline Bi & $/$ & $\boldsymbol{\beta}$ & $\boldsymbol{\beta}$ \\
\hline
\end{tabular}

Fig. 2 Layered crystal structures of group-VA: (a) consisted of parallel puckered layers with space group Cmca (a phase), and (b) consisted of parallel buckled layers with space group R3m (b phase). (c) Stable phases and natural layered crystals of P, As, Sb and Bi crystals.

For bulk antimony, three known allotropes under normal conditions are well known: gray antimony, black antimony and explosive antimony. ${ }^{108}$ Among them, the most stable one is gray antimony. It has the same rhombohedral structure as gray arsenic. Both gray antimony and arsenic show typical semimetal characteristics in their layered bulk forms. In addition, layered gray antimony is also a layered material. Black antimony has the same structure as red phosphorus, and it is formed upon rapid cooling of vapor of antimony. In fact, black antimony has high chemical activity in the atmospheric environment. Under vacuum, black antimony also transforms easily into the stable crystalline gray antimony at approximately $373 \mathrm{~K}$. Explosive antimony often transforms fiercely into gray antimony under mechanical stress or heating. It should be noted that explosive antimony is probably not an allotrope, but a mixed form.

For bulk bismuth, only one stable form exists, which possesses a rhombohedral A7-type structure like gray arsenic and antimony, with a natural layered structure, and layered bismuth shows metallicity. ${ }^{108}$

In summary, the stable phases and natural layered crystals are listed in Fig. 2c. Two stable phases have been isolated, for $\mathrm{P}$ and $\mathrm{As}$, $\mathrm{a}$ and $\mathrm{b}$ phases, whereas there is only one phase, for $\mathrm{Sb}$ and $\mathrm{Bi}$, a b phase. In addition, the natural layered crystals of $\mathrm{As}, \mathrm{Sb}$ and $\mathrm{Bi}$ have $\mathrm{a} b$ phase.

\section{$2.12 \mathrm{D}$ group-VA semiconductors}

The 2D group-VA monolayer materials, derived from phosphorus, arsenic, antimony and bismuth, are called phosphorene, arsenene, antimonene and bismuthene. Akin to group-IVA graphene, silicene, germanene and stanene, these group-VA monolayer materials are termed with suffix "ene", although there are no double bonds. We must state that there are neither nomenclatural reasons for the term "ene" nor linguistic reasons for the suffix "ene" for these monolayer materials. However, in the field of 2D materials, the suffix "ene" is commonly used as an indication of monolayer materials. Therefore, we also adopted this terminology. For simplicity, the emerging monolayer group-VA materials are collectively termed as VAene, which include phosphorene, arsenene, antimonene and bismuthene. Depending on the context, this terminology is also commonly applied to few layer realizations of these materials.

Although initial studies on layered black phosphorus could be dated back to 1914. The renaissance of phosphorene as an elemental 2D material has attracted tremendous interest ever since its first successful 
fabrication in 2014. ${ }^{14-25}$ This new class of layered compounds shares with other 2D materials the typical effects induced by quantum confinement in the vertical direction, but in addition they also might exhibit a rich variety of second-order effects. The group-VA atoms with lone pair electrons alternately stack in an upper and a lower plane in the layered structures, leading to significant puckering and a pseudo-JahnTeller distortion. These two effects result in a size-dependent interlayer interaction, leading to band gap variations and other important properties. Thus, many unique and significant transitions for the electronic and optical properties spring up after the process of thinning the layer thickness to the nanoscale, which are verified in both theoretical and experimental studies. ${ }^{16,26,112}$

Many phosphorene allotropes with compacted honeycomb or nonhoneycomb nanosheets have been designed and studied from first-principles calculations. Zhu et al. predicted a buckled honeycomb phosphorene, called blue phosphorene. ${ }^{113}$ Soon afterwards, Wu et al. reported a much more extended family of phosphorene polymorphs with non-honeycomb structures. ${ }^{114}$ Promisingly, Zhuo et al. further explored stable 2D phosphorus porous polymorphs with tunable electronic properties. ${ }^{115}$ However, the puckered (a-phosphorene) and buckled (b-phosphorene) monolayers are the most common allotropes, which have a structure corresponding to the individual atomic layer of black phosphorus (a phase) and blue phosphorus (b phase) crystals.

As for layered As, Sb, Bi crystals in Fig. 2c, one can clearly see that arsenene may have both puckered and buckled monolayer structures while antimonene and bismuthene are more likely to have a buckled form. As a new 2D group-VA material, it is natural to ask which monolayer structure is the most stable. To address this issue, Zhang et al. systematically predicted a class of group-VA monolayers with five typical honeycomb (a, b, g, d, e) and four non-honeycomb (z, Z, y, i) structures, as illustrated in Fig. $3 \mathrm{a}^{27}$ They took into account their average binding energy for all possible group-VA monolayer configurations, as presented in Fig. 3b. For phosphorene, among the five honeycomb a, b, g, d, e monolayers and four non-honeycomb z, Z, y, i monolayers, a-phosphorene with the puckered form is the most stable. However, different from black phosphorene, for arsenene, antimonene and bismuthene monolayer allotropes, all corresponding $b$ phases with the buckled form have the lowest energy. Note that three phases of bismuthene, namely $\mathrm{a}, \mathrm{b}$ and $\mathrm{z}$, have very close average binding energy. Here, we need to emphasize that the counterpart bulk material of a-phosphorene monolayer is black phosphorus, which is the most stable form of the allotropic bulk crystals under standard conditions. Contrarily, the counterpart bulk materials of $b$-arsenene, $b$-antimonene and b-bismuthene are rhombohedral layered arsenic, gray antimony and bismuth $\mathrm{b}$-phase, respectively. Only $\mathrm{a}$ and $\mathrm{b}$ layered phases experimentally exist in groupVA bulk crystals. In the following text, if no specific explanation is presented, all group-VA monolayers are discussed only based on the two phases, as shown in Fig. 4a.

The phonon spectra of the free-standing group-VA monolayers with the puckered and buckled forms have been calculated based on first-principles calculations, which serve as a criterion to judge the structure stability. As shown in Fig. 4b, no appreciable imaginary phonon modes exist in group-VA monolayers, indicating that these free-standing group-VA monolayers are kinetically stable. In fact, aphosphorene, b-phosphorene, b-arsenene, b-antimonene, a-bismuthene and b-bismuthene have been experimentally prepared and characterized. Regarding the progress in experiments, we will revisit and provide a more detailed description in the following section. 
(a)
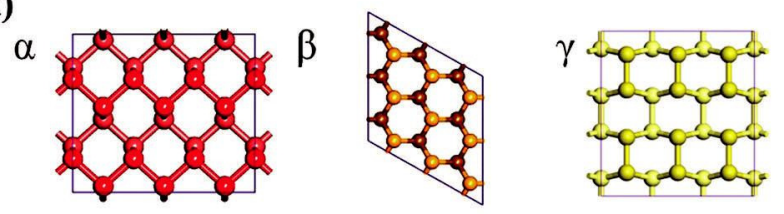

$\delta$

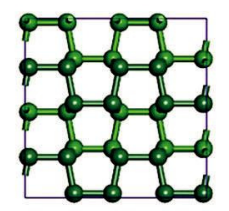

$\varepsilon$
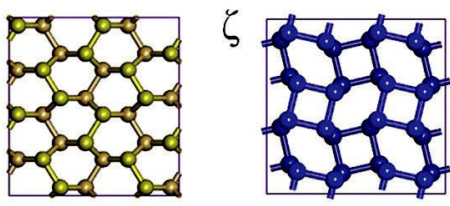

$\eta$

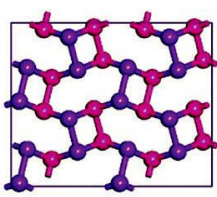

$\theta$
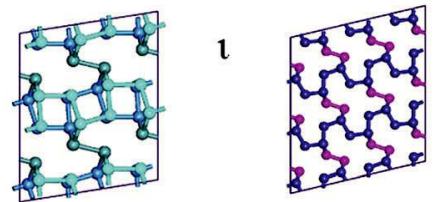

(b)
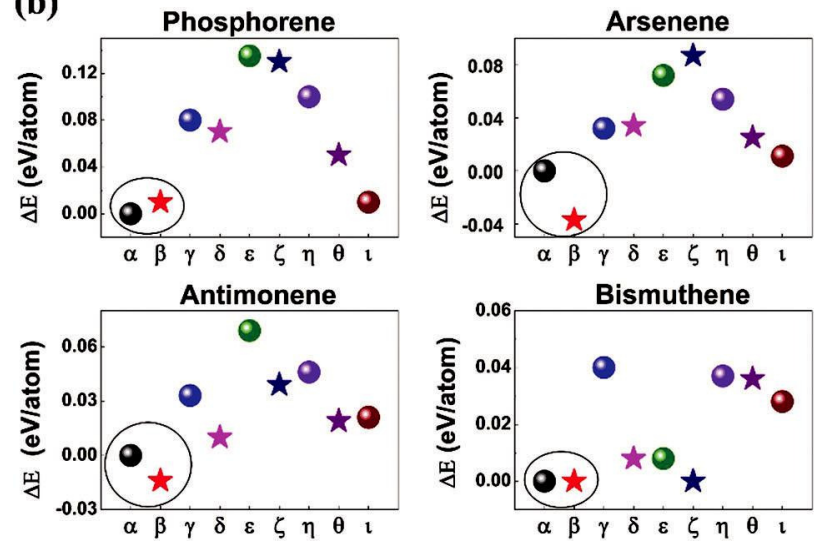

Fig. 3 (a) Top view of the relaxed group-VA (P, As, Sb, Bi) monolayer allotropes with five typical honeycomb structures (a, b, g, d, e) and four non-honeycomb structures (z, Z, y, i). (b) Calculated average binding energies of all group-VA monolayer allotropes, which show energetic stability for $a$ and $b$ phases of group-VA monolayers. Reproduced from ref. 27 with permission from Wiley, copyright 2016.

\subsection{Fundamental properties}

Band structures. To address the promising electronic properties of 2D group-VA materials, many firstprinciples calculations have been performed to study their band structures. Regarding the electronic and optoelectronic applications of 2D group-VA materials, the electronic band gap is one of the most important features in any new 2D materials. Therefore, Table 1 summarizes the calculated band gaps of phosphorene, arsenene, antimonene and bismuthene, based on the Perdew, Burke and Ernzerhof (PBE), ${ }^{116}$ Heyd-Scuseria-Ernzerhof (HSE) hybrid functional, ${ }^{117}$ and the Green's function and screened Coulomb interaction $(\mathrm{GW})$ methods. ${ }^{118,119}$ It should be noted that PBE usually underestimates the band gaps of semiconductors, while HSE and the GW method are more reliable in determining the band gap. Although PBE underestimates the band gap of 2D materials at different levels, it can offer a correct physical picture in agreement with results based on hybrid density functional theory (DFT) and the GW method. 
(a)

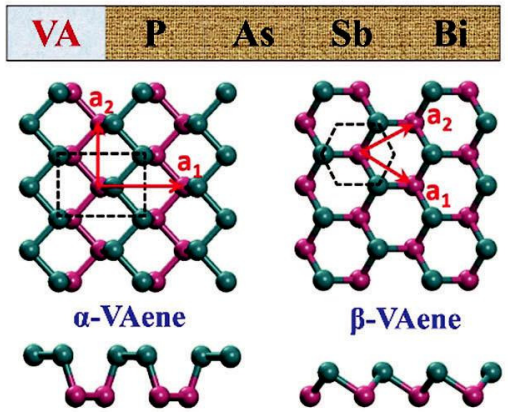

(b)

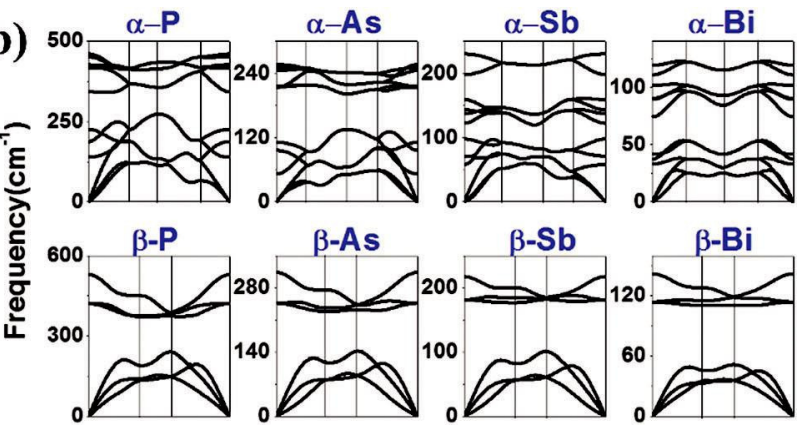

Fig. 4 (a) P, As, Sb and Bi monolayers (collectively termed VAene) for a and b phases. (b) Calculated phonon band dispersions of group-VA monolayers for a and b phases. Reproduced from ref. 27 with permission from Wiley, copyright 2016.

Table 1 Band gaps calculated at different levels of group-VA monolayers for a and $\mathrm{b}$ phases (in units of $\mathrm{eV}$ )

\begin{tabular}{|c|c|c|c|c|}
\hline 2D materials & GGA/LDA & HSE & GW & BSE \\
\hline$\overline{a-P}$ & $0.9,{ }^{\mathrm{I} O \mathrm{O}} 0.91^{\mathrm{IJ}}$ & $1.5,{ }^{\mathrm{IOV}} 1.83^{2 \mathrm{~T}}$ & $\begin{array}{l}2.26^{125} \\
2.2^{121} 2.0^{19}\end{array}$ & $\begin{array}{l}1.41^{125} \\
1.4^{121} \\
1.2^{19}\end{array}$ \\
\hline b-P & $2.0^{19}$ & $2.62^{27}$ & & \\
\hline a-As & $0.83,,^{37} 0.77^{125}$ & $1.66^{27}$ & & \\
\hline b-As & $\begin{array}{l}1.5,{ }^{44} 1.64^{3 \prime} \\
1.6,{ }^{45} 1.76^{66}\end{array}$ & $\begin{array}{l}2.2,^{44} 2.49^{-1} \\
2.0\end{array}$ & $2.47^{\circ 0}$ & $1.6^{\mathrm{oo}}$ \\
\hline $\mathrm{a}-\mathrm{Sb}$ & $\begin{array}{l}0.54(\text { soc })^{43} \\
0.37^{1 \angle د}\end{array}$ & $1.18^{27}$ & & \\
\hline $\mathrm{b}-\mathrm{Sb}$ & $\begin{array}{l}1.65,{ }^{\circ 0} 0.76(\mathrm{soc})^{<0} \\
1.04(\mathrm{soc}),{ }^{\circ 0} \\
1.18(\mathrm{soc})\end{array}$ & $1.55(\mathrm{soc}),{ }^{30} 2.28^{L \prime}$ & $2.38^{\mathrm{oo}}$ & $1.5^{\mathrm{oo}}$ \\
\hline $\mathrm{a}-\mathrm{Bi}$ & $0.16^{125}$ & $0.36^{27}$ & & \\
\hline b-Bi & $\begin{array}{l}0.16,{ }^{1 \angle \mathrm{U}} 0.17(\mathrm{soc})^{1 \angle \mathrm{U}} \\
0.49^{1 \angle \mathrm{J}} \\
0.55,{ }^{1<\mathrm{O}} 0.51(\mathrm{soc})^{1<\mathrm{O}} \\
0.56,{ }^{127} 0.50(\mathrm{soc})^{127}\end{array}$ & $\begin{array}{l}0.14{ }^{1<\mathrm{UO}} 0.03(\mathrm{soc})^{1 \mathrm{LO}} \\
0.99^{2 \prime} \\
0.80,{ }^{1<\mathrm{O}} 0.32(\mathrm{soc})^{1<\mathrm{O}}\end{array}$ & & \\
\hline
\end{tabular}

Black phosphorus is a typical direct-band-gap semiconductor. When thinned to few-layer and monolayer, it still retains a direct band gap, as shown in Fig. 5a. Promisingly, the band gap of phosphorene depends sensitively on the number of layers (Fig. 5b). ${ }^{15}$ The fundamental band gap of semiconductor is dominated by the electron-electron interaction, which can be accurately described by HSE06 or the GW method. Here, we need to stress that the computed results based on the GW method have given a reliable tunable bandgap from 0.3 to $2 \mathrm{eV}$ when the thickness of black phosphorus decreases from bulk to monolayer, which is consistent with the experimental results obtained from Scanning Tunneling Microscopy (STM) measurements. ${ }^{15,27,120-122}$ Besides, the optical gap of a semiconductor can be predicted by the GW method with the Bethe-Salpeter equation (BSE) by considering the excitonic effect. $^{123}$ By means of the GW-BSE method, the estimated exciton binding energy of phosphorene is about 0.8 to $0.9 \mathrm{eV}$, which agrees well with the experimental results. ${ }^{19,112,123}$ In addition, considering that the phosphorus atom is heavier than the carbon atom, the spin-orbital coupling (SOC) of phosphorene has also been taken into account. The calculated results show that including the SOC term has no appreciable effect on the electronic band structures. 
Besides black phosphorene, Zhu et al. predicted another 2D allotrope of phosphorene, namely blue phosphorene. ${ }^{113}$ Blue phosphorene has comparable cohesive energy with black phosphorene and the calculations indicate that it is both dynamically and thermodynamically stable, thus very likely to exist. Based on DFT calculations, blue phosphorene is a semiconductor with an indirect fundamental band gap of approximately $2.0 \mathrm{eV}$, much larger than that of black phosphorene. The band-gap value can be effectively tuned from $2.0 \mathrm{eV}$ to below $0.8 \mathrm{eV}$ via strain, as well as the number of layers. Furthermore, 2D blue phosphorene was successfully grown on $\mathrm{Au}(111)$ in experiments. ${ }^{124}$ The fundamental band gap of the synthesized structures was determined to be $1.10 \mathrm{eV}$ by scanning tunneling spectroscopy (STS) measurements. The discrepancy between theoretical prediction and experimental observation is likely due to the quality of synthesized blue phosphorene and the substrate effect.

Promisingly, Zhang et al. firstly predicted an abrupt transition from semimetallic or metallic As and Sb bulk crystals to wide-band-gap semiconductor arsenene and antimonene monolayers. ${ }^{26}$ Meanwhile, they further found that arsenene and antimonene undergo an indirect-to-direct band-gap transition upon tensile strain. Whereafter, Zhang et al. proposed that $\mathrm{b}$ phases with buckled forms are the most stable structures among the possible arsenene, antimonene and bismuthene allotropes. ${ }^{27}$ Therefore, we discuss the electronic properties of arsenene and antimonene with $b$ phases firstly. Through first-principles theory, $b$ phases of arsenene and antimonene monolayers hold 1.76 and $1.65 \mathrm{eV}$ indirect-band-gaps at the PBE level in Fig. 5c. Based on the GW method, calculated results describe more reliable fundamental band gaps for arsenene and antimonene, which are predicted to be 2.47 and $2.38 \mathrm{eV}{ }^{66}$ The exciton binding energy, given by the difference between the electronic and optical band gaps, is found to be $0.9(0.8) \mathrm{eV}$ for arsenene (antimonene). Considering that antimonene is a heavy element of the fifth period, bantimonene may possess a strong SOC effect. The band gap of b-antimonene with SOC is $1.04 \mathrm{eV}$ at the PBE level and $1.55 \mathrm{eV}$ at the HSE level, while also in the presence of an indirect bandgap. Besides, the calculated imaginary part of the dielectric functions for arsenene and antimonene monolayers, in Fig. 5d, shows that the optical absorption occurs at 1.6 (1.5) eV within the red wavelength for arsenene (antimonene). ${ }^{66}$ 

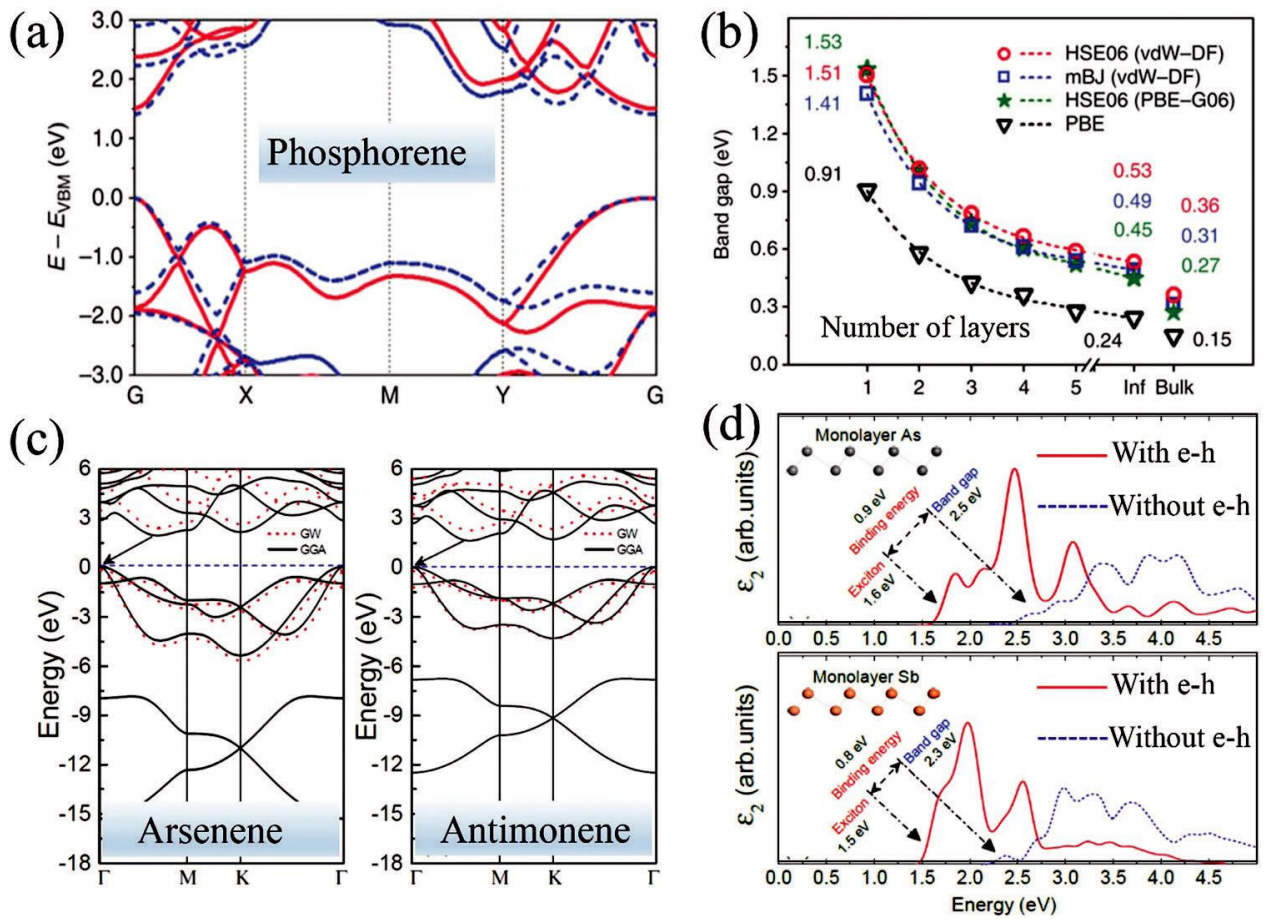

Fig. 5 (a) Band structures of black phosphorene monolayer calculated with the HSE06 functional (red solid lines) and the mBJ potential (blue dashed lines), respectively. (b) Evolution of the direct bandgaps as a function of the sample thickness. Functionals used for structural optimization are shown in parentheses. (a and b) Reproduced from ref. 15 with permission from Nature Publishing Group, copyright 2014. (c) Band structures of arsenene and antimonene monolayers calculated with the PBE and GW approaches, respectively. (d) Calculated imaginary part of the dielectric function $e_{2}$ for arsenene and antimonene monolayers with and without the electron-hole $(\mathrm{e}-\mathrm{h})$ interaction. (c and d) Reproduced from ref. 66 with permission from the American Chemical Society, copyright 2017.

As the last element of group-VA, a layered b-bismuth crystal is characterized by a small density of states around the Fermi level, showing a semi-metallic property, while its single-layer structure is a narrow band gap semiconductor. ${ }^{125-128}$ Because bismuth is heavier than other group-VA elements, stronger SOC should affect the band structure of bismuthene. Aktu"rk et al. calculated the electronic structures of bismuthene with and without SOC by means of the PBE and HSE functionals. ${ }^{126}$ The HSE correction applied to PBE states increases the direct band gap to $0.80 \mathrm{eV}$. However, when SOC is included, this fundamental band gap decreases to $0.32 \mathrm{eV}$ and the band structure transforms from direct band gap into indirect band gap.

Besides the $\mathrm{b}$ phase, a phases of arsenene, antimonene and bismuthene also exhibit good thermodynamic stability in spite of their metastable phases. They have band gaps of $0.77,0.37$ and 0.16 $\mathrm{eV}$ based on the PBE method, and 1.66, 1.18 and $0.99 \mathrm{eV}$ based on the HSE correction. ${ }^{27,125}$ Among them, a-bismuthene shows a direct band gap and it also turns into an indirect band gap semiconductor with the inclusion of SOC, just like b-bismuthene. ${ }^{125-128}$ Notably, a phases of arsenene, antimonene and bismuthene hold much smaller band gaps than the most stable b phases, respectively.

Carrier transport. 2D group-VA semiconductors exhibit superior carrier transport properties, leading to their promising application in electronic and optoelectronic devices. It is known that carrier transport properties are evaluated based on the calculation of mobility. In theory, the carrier mobility of 2D materials is defined by the following expression: ${ }^{15}$ 


$$
\mu_{2 D}=\frac{2 e \hbar^{3} C_{2 D}}{3 k_{B} T\left|m^{*}\right|^{2} E_{1}^{2}}
$$

where $\mathrm{h}, \mathrm{k}_{\mathrm{B}}$, and $\mathrm{T}$ are the reduced Planck constant, Boltzmann constant and temperature, respectively. The term $\mathrm{m}^{*}$ is the effective mass along the transport direction and $\mathrm{C}_{2 \mathrm{D}}$ means the elastic modulus. $\mathrm{E}_{1}$ represents the deformation potential constant of the valence-band minimum (hole) or conduction-band maximum (electron) along the transport direction. From the definition, it is clear that the carrier mobility relies on three intrinsic properties of $2 \mathrm{D}$ materials, which are effective mass $\mathrm{m}^{*}$, elastic modulus $\mathrm{C}_{2 \mathrm{D}}$ and deformation potential constant $\mathrm{E}_{1}$.

Qiao et al. ${ }^{15}$ predicted that the mobilities of black phosphorene are hole-dominated, anisotropic and rather high based on HSE06 calculations. The electron mobility is $1100-1140 \mathrm{~cm}^{2} \mathrm{~V}^{-1} \mathrm{~s}^{-1}$ along the armchair direction and $\sim 80 \mathrm{~cm}^{2} \mathrm{~V}^{-1} \mathrm{~s}^{-1}$ along the zig-zag direction. In particular, the value of hole mobility along the armchair direction is $640-700 \mathrm{~cm}^{2} \mathrm{~V}^{-1} \mathrm{~s}^{-1}$ and the value along the zigzag direction is extremely high, up to $10000 \mathrm{~cm}^{2} \mathrm{~V}^{-1} \mathrm{~s}^{-1}$. The extraordinarily large hole mobility is much higher than the mobility of $\operatorname{MoS}_{2}\left(\sim 200 \mathrm{~cm}^{2} \mathrm{~V}^{-1} \mathrm{~s}^{-1}\right),{ }^{129}$ mainly benefiting from the extremely small deformation potential $\left(\mathrm{E}_{1}=\right.$ $0.150 .03 \mathrm{eV}$ ). The carrier mobilities of black phosphorene present strongly anisotropic characteristics in virtue of the highly anisotropic atomic structure, which causes the big differences in effective mass (Fig. 6a), elastic modulus and deformation potential constant along armchair and zigzag directions. Additionally, the high in-plane transport anisotropy of black phosphorene distinguishes it from other typical 2D materials, such graphene, silicene, h-BN and so on.

The carrier transport properties of arsenene, antimonene and bismuthene are also studied by the deformation potential method. ${ }^{27,36,66,130}$ Based on the method, the carrier mobilities of $a$ and $b$ arsenene, $b$ antimonene, and $\mathrm{b}$ bismuthene are as high as several thousand $\mathrm{cm}^{2} \mathrm{~V}^{-1} \mathrm{~s}^{-1} \cdot{ }^{27,36,130}$ Pizzi et al. ${ }^{36}$ calculated the electron and hole mobilities to be 635 and $1700 \mathrm{~cm}^{2} \mathrm{~V}^{-1} \mathrm{~s}^{-1}$ for $\mathrm{b}$ arsenene, and 630 and $1737 \mathrm{~cm}^{2} \mathrm{~V}^{-1} \mathrm{~s}^{-1}$ for $\mathrm{b}$ antimonene. They emphasized that the SOC effects hardly influence the conduction band of As and Bi but change the topmost valence band, leading to the changes of effective masses and deformation potentials. As a result, the hole mobilities of As and Bi monolayers are increased by $25 \%$ and $84 \%$, respectively. 
(a)

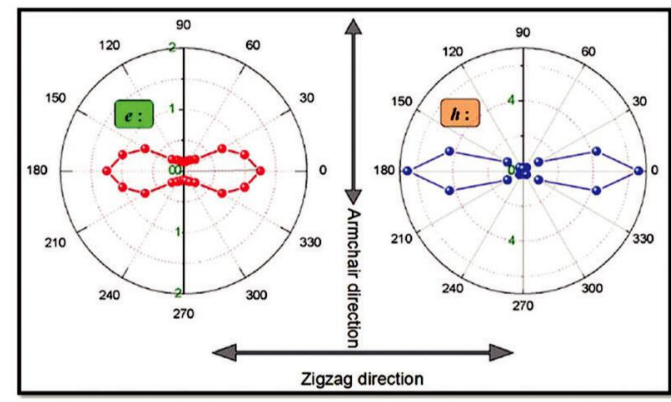

(b)

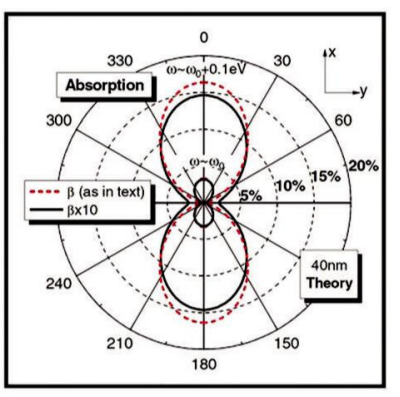

(c) $)^{5}$

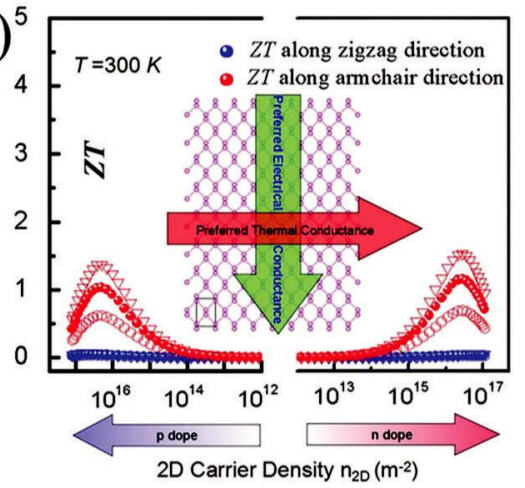

(d)

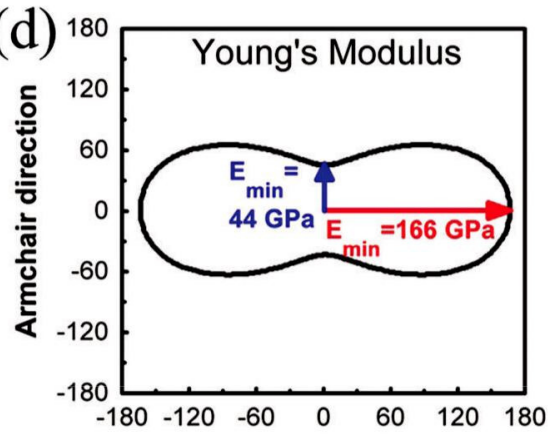

Fig. 6 (a) Effective mass of electrons and holes according to spatial directions. (b) Polar representation of the absorption coefficient A(a) for a $40 \mathrm{~nm}$ intrinsic black phosphorus film for normal incident light with excitation energies at the band gap o, and larger. a is the light polarization angle. A(a) is plotted for two values of interband coupling strengths. Reproduced from ref. 20 with permission from the American Physical Society, copyright 2014. (c) The thermoelectric figure of merit according to the doping density at $\mathrm{T}=$ $300 \mathrm{~K}$. Different long-wave relaxation times of phonons are included. The unit of the doping density is $\mathrm{m}^{2}$. (a and c) Reproduced from ref. 139 with permission from the American Chemical Society, copyright 2014. (d) Direction dependence of Young's modulus of phosphorene. Reproduced from ref. 140 with permission from the American Institute of Physics, copyright 2014.

Optical properties. 2D group-VA materials present unique optical properties which extend their potential applications. Qiao et al. ${ }^{15}$ and Tran et al. ${ }^{19}$ predicted the optical adsorption spectrum of black phosphorene by first-principles calculations. The calculated absorption spectrum is anisotropic, for light linearly polarized in the armchair and zigzag directions. The band edge of the first absorption peak is found at the bandgap and falls quickly with increasing layer thickness in the armchair direction, while the peak is found at $3.14 \mathrm{eV}$ in the monolayer and falls slightly with thickness in the zigzag direction. This in-plane linear dichroism allows optical determination of the crystalline orientation and optical activation of the anisotropic transport properties. Tran et al. ${ }^{19}$ calculated that the phosphorene monolayer absorbs light between 1.1 and $2.8 \mathrm{eV}$ along the armchair direction and is transparent to light in the same energy range along the zigzag direction, covering the infrared and a part of the visible light regime of broad interest. Moreover, the polarization energy window is tunable over a wide range by adjusting the layer stacking number, which is promising for device design. By using the Kubo formula within an effective low-energy Hamiltonian, Low et al. ${ }^{20}$ investigated the optical conductivity tensor of black phosphorene, which can express physical quantities observed in optical experiments (Fig. 6b). The optical conductivity of black phosphorene, similar to the optical adsorption spectrum, is very sensitive to the number of layers, especially for frequencies ranging from 2500 to $5000 \mathrm{~cm}^{-1}$.

By calculating dielectric functions, electron energy loss spectra, absorption coefficients, refractive indices, and optical reflectivities in the $0-21 \mathrm{eV}$ energy range, Singh et al. ${ }^{43}$ studied the optical properties 
of antimonene. They found that it is suitable for ultraviolet optical nanodevices, microelectronic devices and solar cells. The dielectric functions become negative between $5.1 \mathrm{eV}$ and $9.0 \mathrm{eV}$ for a-antimonene and between $6.9 \mathrm{eV}$ and $8.4 \mathrm{eV}$ for $\mathrm{b}$-antimonene, indicating their metallic character in the UV part of the electromagnetic spectrum. In addition, the result that electron energy loss spectra reveal the plasmon energies of around $9 \mathrm{eV}$ also suggests its metallic behavior regarding the refection of light. The values of refractive indices are 2.3 for a-antimonene and 1.5 for b-antimonene at the zero energy limit and scale up to 3.6 in the ultraviolet region.

Thermal properties. Thermal properties of $2 \mathrm{D}$ group-VA materials have been widely studied by theoretical and experimental methods. ${ }^{48,128,131-138}$ The anisotropic phonon property in a phases of phosphorene, arsenene, antimonene and bismuthene brings about an asymmetrical thermal conductivity and thermoelectric efficiency. ${ }^{131-135}$ Aierken et al. ${ }^{131}$ calculated the thermal properties of black and blue phosphorene through first-principles calculations. They predicted that the linear thermal expansion coefficients of black phosphorene along the zig-zag and armchair directions are highly anisotropic (up to $20 \%$ ), while the blue phase is isotropic in thermal expansion. Zhu et al. ${ }^{135}$ found that thermal conductivities in black phosphorene are highly anisotropic because of orientation dependent group velocities and phonon relaxation times. Their theoretical calculation indicated that thermal conductivities at $300 \mathrm{~K}$ are 83.5 and $24.3 \mathrm{~W} \mathrm{~m}^{-1} \mathrm{~K}^{-1}$ along zigzag and armchair directions, respectively. More interestingly, the electronic conductivity along the armchair direction is much larger than that along the zigzag direction. ${ }^{139}$ Therefore, thermoelectric figure of Merit (ZT), dependent not only on thermal conductivity but also on electronic conductivity, is also highly anisotropic for black phosphorene, where the value along the armchair direction is larger than that along the zigzag direction (Fig. 6c). ${ }^{139}$ The intrinsic value of ZT in phosphorene is unfriendly low but can be increased with different enhanced methods, which will be described in detail later. By using first-principles anharmonic lattice dynamics calculations and the Boltzmann transport equation for phonons, Zeraati et al. ${ }^{48}$ investigated the phonon dispersion and the lattice thermal conductivity of a-arsenene. Results show that arsenene presents a smaller and more anisotropic thermal conductivity than phosphorene. The thermal conductivities of arsenene along the zigzag and armchair directions are 30.4 and $7.8 \mathrm{~W} \mathrm{~m}{ }^{1} \mathrm{~K}^{1}$ at room temperature, respectively. Due to the puckered structure, the thermal conductivity of arsenene is mainly provided by the longitudinal acoustic phonon modes at temperatures above $100 \mathrm{~K}$.

The buckled structure of group-VA materials reveals isotropic characteristic for thermal properties. Wang et al. ${ }^{136}$ predicted that antimonene has a low lattice thermal conductivity of $15.1 \mathrm{~W} \mathrm{~m}^{1} \mathrm{~K}^{1}$ at 300 $\mathrm{K}$, accounting for its small group velocity, low Debye temperature and large buckling height. The low thermal conductivity can be tuned to be smaller by minimizing the sample size and chemical functionalization. ${ }^{136,137}$ The intrinsic values of thermoelectric figure for antimonene and bismuthene are not sufficiently high for potential application but can be effectively increased by the $\mathrm{n} / \mathrm{p}$-doping method. ${ }^{128,138}$

Mechanical properties. Due to the puckered and buckled structures, 2D group-VA materials exhibit distinctive mechanical properties. Black phosphorene has special and anisotropic mechanical properties, as depicted in Fig. 6d. ${ }^{24,140,141}$ Wei et al. ${ }^{140}$ used first-principles calculations to find that the maximal Young's modulus of $166 \mathrm{GPa}$ is along the zigzag direction and the minimal value of $44 \mathrm{GPa}$ is along the armchair direction (Fig. 6d). Along the armchair direction black phosphorene can suffer a strain of up to $30 \%$. In particular, Jiang et al. ${ }^{24}$ reported a negative Poisson's ratio in the out-of-plane direction during the deformation of phosphorene in its zigzag direction, originating from its puckered structure. This fantastic property endows phosphorene with great potential to be an auxetic material. Moreover, theoretical research studies verified that a arsenene and bismuthene also have directional elastic behaviors. ${ }^{126,142}$ The in-plane stiffness values of As are $C_{x}=20 \mathrm{~N} \mathrm{~m}^{-1}$ and $C_{y}=55 \mathrm{~N} \mathrm{~m}^{-1}$, while the values of $B i$ are $C_{x}=8.04 \mathrm{~N} \mathrm{~m}^{-1}$ and $C_{y}=22.6 \mathrm{~N} \mathrm{~m}^{-1}$. Clear anisotropic Poisson's ratios for As and Bi 
are observed, as $\mathrm{n}_{\mathrm{xy}}=0.33$ and $\mathrm{n}_{\mathrm{yx}}=0.91$ for As, $\mathrm{n}_{\mathrm{xy}}=0.33$ and $\mathrm{n}_{\mathrm{yx}}=0.93$ for Bi. Along the zig-zag direction the values of $\mathrm{n}_{\mathrm{yx}}$ are rather high both for As and Bi. Importantly, in-plane stiffness influences the robustness of the structure while Poisson's ratio is meaningful to the variation of the electronic structure, in particular for possible metal-insulator transitions under tensile strain.

As for mechanical properties, theoretical calculations ${ }^{126,142}$ show that the in-plane stiffness values of $\mathrm{b}$ arsenene and bismuthene are isotropic, that is $\mathrm{C}=58 \mathrm{~N} \mathrm{~m}^{-1}$ and $23.9 \mathrm{~N} \mathrm{~m}^{-1}$. The isotropic Poisson's ratios of $\mathrm{As}$ and $\mathrm{Bi}$ have a uniform value of 0.21 and 0.33 , respectively. All the values of in-plane stiffness are calculated to be much smaller than those of some typical honeycomb materials, such as graphene $\left(330 \mathrm{~N} \mathrm{~m}^{-1}\right)$ and h-BN $\left(240 \mathrm{~N} \mathrm{~m}^{-1}\right)$.

\subsection{Tunable electronic properties}

The tunability of the material properties in such 2D group-VA nanosheets offers a charming prospect of engineering versatile applications. Various strategies such as strain, electric field, doping, defect, chemical functionalization, heterostructure have been theoretically proposed to effectively tune their fundamental properties, as shown in Fig. 7 and 8. Strain/electric field. It is well known that ultrathin 2D materials often hold high mechanical stretchability, and can reversibly withstand extreme structural deformation. Therefore, strain has been considered as an effective method to modulate electronic properties of 2D materials, and can further extend their applications (Fig. 7a-e). ${ }^{132,143,144}$ Peng et al. investigated the band gap of phosphorene under external strain. ${ }^{144}$ They found that its band gap is sensitive to strain. As is illustrated in Fig. 7b, when a compressive strain is applied along the zig-zag direction of phosphorene, the value of band gap decreases with the increase of strain. Intriguingly, the value of band gap increases to a maximum with critical tensile strain (4\%) in the zig-zag direction and then decreases with increasing strain. The same trend of change also appears when the external strain is along the armchair direction. Moreover, the effective mass of phosphorene also causes the dramatic transformation under external strain. Fei and Yang found that the favorable direction of electron transport can be switched by the applied biaxial strain, as shown in Fig. $7 d .{ }^{14}$ Furthermore, the electron mobility along the zig-zag direction is much larger than that along the armchair direction, while the hole mobility is not sensitive to the applied strain. Moreover, an electric field can also tune the electronic properties of phosphorene. Dai and Zeng studied the band gap of few-layer phosphorene under an external electric field. ${ }^{145}$ The band gap decreases (from 0.78 to $0.56 \mathrm{eV}$ ) with increasing electric field while monolayer phosphorene only has a tiny change (from 0.86 to $0.78 \mathrm{eV}$ ). Interestingly, researchers also reported the possibility of achieving a topological state by an external electric field that induces a specific band inversion. $^{17}$ 


\section{(a)}

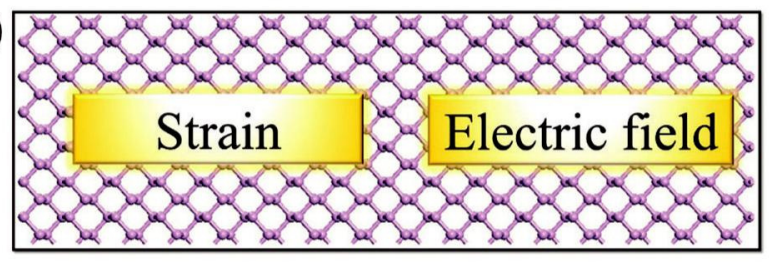

(b)
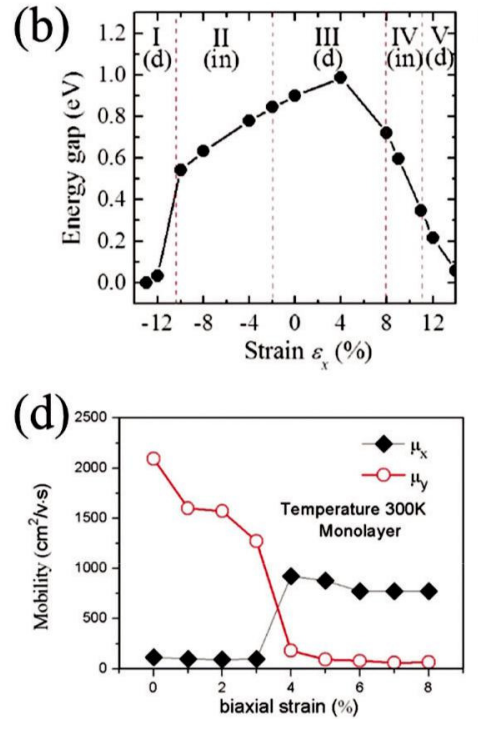

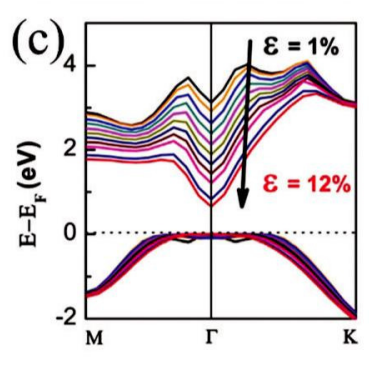

(e)

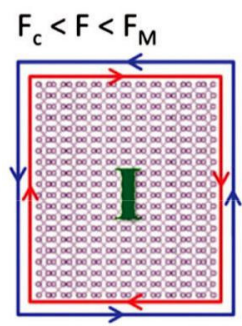

Fig. 7 (a) Schematic of strain and electric field modulating the electronic properties of group-VA monolayers. (b) The band gap of 2D phosphorene as a function of strain $\mathrm{e}_{\mathrm{x}}$ applied in the zig-zag direction. Five strain zones were identified for $\mathrm{e}_{\mathrm{x}}$ based on its distinct band structures. Zones I, II, III, IV and V correspond to the direct (d), indirect (in), direct, indirect, and direct gaps, respectively. The critical strains for the gap transition are $10.2 \%, 2 \%,+8 \%$ and $+11.3 \%$. The gap closes up at $\mathrm{e}_{\mathrm{x}}=13 \%$. Reproduced from ref. 144 with permission from the American Physical Society, copyright 2014. (c) Changes in the valence-band top and the conduction-band bottom with increasing biaxial tensile strain. Reproduced from ref. 26 with permission from Wiley, copyright 2015. (d) Electron mobilities of monolayer phosphorene for biaxial strain at room temperature. Reproduced from ref. 14 with permission from the American Chemical Society, copyright 2014. (e) Schematic band structure and spin and charge current in the phosphorene channel for $F_{c} \circ \mathrm{F} o F_{M}$. The letter "I" stands for insulating. Reproduced from ref. 17 with permission from the American Chemical Society, copyright 2015.

For other 2D group-VA materials, analogous properties are also found by first-principles computations. Zhang et al. found that both arsenene and antimonene experience a significant indirect-todirect band gap transition under small biaxial strain. ${ }^{26}$ Here, taking arsenene as an example, under $0-3 \%$ biaxial strain, the conduction band minimum of the buckled arsenene remains in the Brillouin zone halfway between the $\mathrm{G}$ and $\mathrm{M}$ high-symmetry points. However, further increase of tensile strain up to $4 \%$ can rapidly change the indirect-band-gap buckled arsenene into a direct-band-gap semiconductor. Importantly, in the scope of tensile strain from $4 \%$ to $12 \%$, arsenene maintains its semiconducting property with a direct band gap (Fig. 7c). The overall trend for the electronic band characters of antimonene in response to biaxial tensile strain is quite similar to that of arsenene. Thus, a change from an indirect to a direct band-gap semiconductor is observed in both arsenene and antimonene. Consequently, the direct band structures of strained arsenene and antimonene have a clear advantage for potential application in optical devices, because electronic excitation becomes feasible with lower phonon energies.

In addition to the modulation of the electronic band structures of arsenene and antimonene, strain has also been implemented to induce a topological insulator transition. ${ }^{40,47}$ Many theoretical studies 
demonstrated that strain could drive the normal insulators of arsenene and antimonene to novel topological nontrivial 2D materials with a non-zero Chern number (Fig. 7e).

Defect. Theoretical calculations have revealed that defects can effectively affect the electronic properties of 2D group-VA materials and produce magnetism in nonmagnetic pristine phosphorene, arsenene and antimonene (Fig. 8a). ${ }^{146-154}$ Hong et al. ${ }^{148}$ studied the electronic band structures and bias dependent transport properties of various defective phosphorene systems. They found that the band gap closes in single vacancy phosphorene, but reappears in the divacancy system. Moreover, the vacancy defects can greatly increase the phosphorene-based device current. Hu et al. ${ }^{147}$ studied ten kinds of point defects in phosphorene, including Stone-Wales defects, single vacancies (SV) and double vacancies (DV), as listed in Table 2. Among these defects of phosphorene, the SV-(5|9) and DV-(5|8|5)-2 defects can generate unoccupied localized states in the fundamental band gap. Besides, the SV-(519) and SV(55|66) defects can result in hole doping, and the SV-(5|9) defect induces local magnetic moments. Typical point defects in arsenene and antimonene are also investigated systematically by Sun et al. ${ }^{153}$ (Table 2). They found that most of the defective configurations maintain indirect band gaps with reduced band gap values. In particular, the 5551777 defect in antimonene brings a transition from an indirect band gap to a direct band gap. Most SV defects carry magnetic moments due to the dangling bonds. In addition, Yang et al. ${ }^{154}$ found that a single vacancy in antimonene makes it metallic but its four divacancies preserve the semiconducting character with the reduced bandgap.

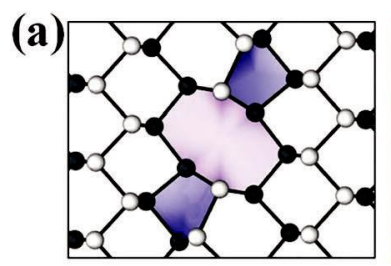

(c)
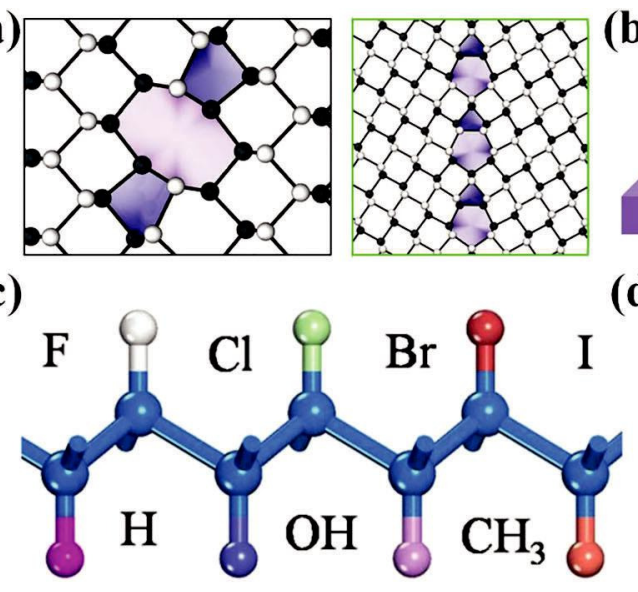

(b)

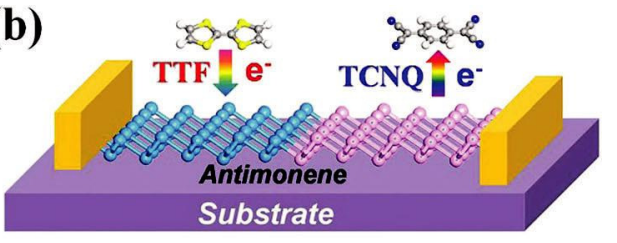

(d)

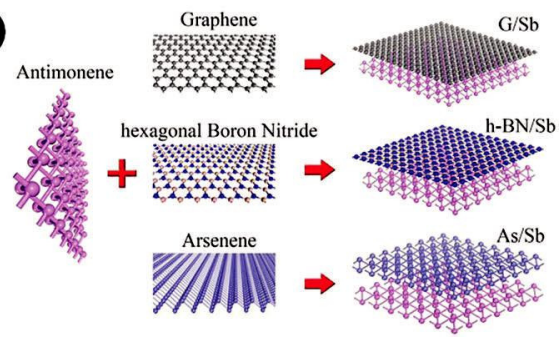

Fig. 8 (a-d) Schematic illustrations of group-VA materials with defect, doping, chemical functionalization, and heterostructures. (a) Reproduced from ref. 146 with permission from the American Chemical Society, copyright 2014. (b and d) Reproduced from ref. 175 and 207 with permission from the Royal Society of Chemistry, copyright 2016. 
Table 2 Calculated formation energies $\mathrm{E}_{\mathrm{f}}\left(\mathrm{eV}\right.$ per atom) and band gaps $\mathrm{E}_{\mathrm{g}}(\mathrm{eV})$ of perfect and defective phosphorene $(\mathrm{a}-\mathrm{P}),{ }^{147}$ arsenene $(\mathrm{b}-\mathrm{As})^{153}$ and antimonene $(\mathrm{b}-\mathrm{Sb})^{153}$

\begin{tabular}{|c|c|c|c|}
\hline 2D systems & Defect & $\mathrm{E}_{\mathrm{f}}(\mathrm{eV})$ & $\mathrm{E}_{\mathrm{g}}(\mathrm{eV})$ \\
\hline $\mathrm{a}-\mathrm{P}$ & $\begin{array}{l}\text { Perfect } \\
\text { SW-1 } \\
\text { SW-2 } \\
\text { SV-(5|9) } \\
\text { SV-(55|66) } \\
\text { DV-(5|8|5)-1 } \\
\text { DV-(5|8|5)-2 } \\
\text { DV-(4|10|4) } \\
\text { DV-(555|777)-1 } \\
\text { DV-(555|777)-2 } \\
\text { DV-(555|777)-3 }\end{array}$ & $\begin{array}{l}0.000 \\
1.012 \\
1.322 \\
1.626 \\
2.025 \\
1.906 \\
3.041 \\
2.137 \\
2.081 \\
2.350 \\
2.613\end{array}$ & $\begin{array}{l}0.905 \\
0.928 \\
0.883 \\
0.190 / 0.941 \\
\text { Hole doping } \\
0.962 \\
0.559 \\
0.929 \\
0.966 \\
0.962 \\
0.973\end{array}$ \\
\hline b-As & $\begin{array}{l}\text { Perfect } \\
\text { SW } \\
\text { SV } \\
51815 \\
5551777\end{array}$ & $\begin{array}{l}0.00 \\
1.33 \\
2.13 \\
2.37 \\
1.96\end{array}$ & $\begin{array}{l}1.60 \\
1.29 \\
0.09 \\
0.99 \\
1.44\end{array}$ \\
\hline $\mathrm{b}-\mathrm{Sb}$ & $\begin{array}{l}\text { Perfect } \\
\text { SW } \\
\text { SV } \\
51815 \\
5551777\end{array}$ & $\begin{array}{l}0.00 \\
1.09 \\
1.86 \\
2.08 \\
1.09\end{array}$ & $\begin{array}{l}1.26 \\
0.96 \\
0.00 \\
0.74 \\
1.18\end{array}$ \\
\hline
\end{tabular}

Surface functionalization. Surface functionalization is an effective strategy to modify intrinsic properties of 2D group-VA materials through physical adsorption and chemical functionalization.

Physical adsorption behavior of 2D group-VA materials has been widely studied based on firstprinciples calculations (Fig. 8b). Physical adsorption includes the doping of metallic atoms ( Na, K, Li, Al, and so on), nonmetallic atoms $(\mathrm{H}, \mathrm{C}, \mathrm{F}, \mathrm{Cl}$,

and so on), and small molecules (gas molecules and organic molecules). ${ }^{155-175}$ Table 3 summarizes the modification of electronic properties by some metallic and nonmetallic atoms in 2D group-VA materials.

Tunable electronic properties have been realized in phosphorene and arsenene by external adsorption doping. Using means of selective doping, one can transform the semiconducting a-phosphorene and barsenene into a half-metal, even a metal, or transform a spin-non-polarized semiconductor into a spinpolarized semiconductor. For example, adsorption of B, C, N, F, Li, Na, K, Al and Cr on phosphorene induces a fermi level shift and produces metallic electronic structures. ${ }^{155,160,161}$ For arsenene, V, Mn, Fe and $\mathrm{Ni}$ doped systems possess half-metallic properties, while $\mathrm{C}, \mathrm{O}, \mathrm{Se}$, Ti and $\mathrm{Zn}$ doped systems present metallic states. ${ }^{57,167,168}$ Additionally, transition metal (Ti, V, Cr, Mn, Fe, Co) doping can induce magnetism in a-phosphorene. ${ }^{151,160-163}$ Besides transition metal doping, H, B, C, O, Si, S, Ge, Sn, NO and $\mathrm{NO}_{2}$ can also produce magnetic states in arsenene. ${ }^{57,166-173}$

Kou et al. ${ }^{159}$ investigated the adsorption of $\mathrm{CO}, \mathrm{CO}_{2}, \mathrm{NH}_{3}, \mathrm{NO}$ and $\mathrm{NO}_{2}$ gas molecules on phosphorene by first-principles calculations. Their results implied that the $\mathrm{CO}$ and $\mathrm{CO}_{2}$ adsorbed on phosphorene systems have small adsorption energies, and the adsorption of $\mathrm{CO}, \mathrm{CO}_{2}$ and $\mathrm{NH}_{3}$ doesn't exert a substantial effect on its electronic structures. On the other hand, the adsorption of $\mathrm{NO}$ and $\mathrm{NO}_{2}$ presents a higher adsorption energy. Both $\mathrm{NO}$ and $\mathrm{NO}_{2}$ adsorption result in a magnetic moment of $1 \mathrm{~m}_{\mathrm{B}}$. In addition, the $\mathrm{NH}_{3}$ (NO) adsorption on phosphorene can alter the resistance and lead to the increase (decrease) of the current by transport calculations. Zhang et al. ${ }^{158}$ studied two kinds of organic molecules adsorbed on phosphorene, an electrophilic molecule tetra-cyano-p-quinodimethane (TCNQ) as an electron acceptor and a nucleophilic molecule tetrathiafulvalene (TTF) as an electron donor. After TCNQ or TTF 
adsorption, the band gap decreases because of their impurity states. The TCNQ adsorption can lead to a typical p-type phosphorene with considerable electron transfer from phosphorene to TCNQ. The phosphorene adsorbed by a typical nucleophilic molecule TTF does not become effectively n-doped, but the doping states can be strengthened by an external electric field or strain.

Table 3 Calculated adsorption energy $E_{a}(e V)$ and local magnetic moment $m\left(m_{B}\right)$ for an adatom adsorbed on aphosphorene, ${ }_{155} \mathrm{~b}$-arsenene ${ }^{166}$ and $b$-antimonene. ${ }^{39}$ The band gap $E_{g}(e V)$ for an adatom adsorbed on aphosphorene. ${ }^{155}$ The charge transfer $\mathrm{r}(\mathrm{e})$ between the adatom and $\mathrm{b}$-arsenene $\mathrm{e}^{166} / \mathrm{b}$-antimonene $\mathrm{e}^{39}$

\begin{tabular}{|c|c|c|c|c|c|c|c|}
\hline \multicolumn{4}{|c|}{ a-Phosphorene } & \multicolumn{4}{|c|}{ b-Arsenene } \\
\hline \multicolumn{4}{|c|}{$\mathrm{E}$} & & & & \\
\hline Adatom & $\mathrm{a}$ & $\mathrm{m}$ & $E_{g}$ & Adatom & $\mathrm{E}_{\mathrm{a}}$ & $\mathrm{m}$ & $\mathrm{r}$ \\
\hline B & 5.08 & 1.00 & - & $\mathrm{H}$ & 1.185 & 1.0 & 0.84 \\
\hline $\mathrm{C}$ & 5.16 & 0.00 & - & $\mathrm{Li}$ & 1.720 & 0.0 & +0.19 \\
\hline $\mathrm{N}$ & 2.98 & 1.00 & - & B & 4.511 & 1.0 & 0.84 \\
\hline $\mathrm{O}$ & 4.69 & 0.00 & - & $\mathrm{C}$ & 4.398 & 2.0 & 1.32 \\
\hline $\mathrm{F}$ & 2.30 & 1.00 & - & $\mathrm{N}$ & 1.650 & 0.0 & 1.14 \\
\hline $\mathrm{Li}$ & 1.823 & 0.00 & 0.00 & $\mathrm{O}$ & 4.130 & 0.0 & 1.07 \\
\hline $\mathrm{Na}$ & 1.291 & 0.00 & 0.00 & $\mathrm{Al}$ & 1.598 & 0.0 & +0.34 \\
\hline $\mathrm{Mg}$ & 0.613 & 0.00 & 0.54 & $\mathrm{Si}$ & 2.233 & 0.0 & 0.10 \\
\hline $\mathrm{Al}$ & 1.988 & 0.00 & 0.00 & $\mathrm{P}$ & 1.812 & 1.0 & 1.13 \\
\hline $\mathrm{Cr}$ & 1.721 & 4.89 & 0.00 & $\mathrm{Cl}$ & 1.799 & 1.0 & 0.48 \\
\hline $\mathrm{Fe}$ & 3.129 & 2.00 & 0.68 & $\mathrm{Ti}$ & 3.405 & 0.0 & 0.75 \\
\hline Co & 4.381 & 1.00 & 0.26 & $\mathrm{Ga}$ & 1.570 & 0.0 & 0.08 \\
\hline $\mathrm{Ni}$ & 4.482 & 0.00 & 0.89 & $\mathrm{Ge}$ & 1.622 & 2.0 & +0.18 \\
\hline Mo & 3.305 & 0.00 & 0.93 & As & 1.492 & 1.0 & +0.13 \\
\hline $\mathrm{Pd}$ & 3.505 & 0.00 & 0.95 & $\mathrm{Se}$ & 2.428 & 0.0 & 0.21 \\
\hline $\mathrm{Pt}$ & 4.842 & 0.00 & 0.94 & $\mathrm{Sb}$ & 1.028 & 1.0 & 0.02 \\
\hline $\mathrm{Au}$ & 1.636 & 1.00 & 0.08 & & & & \\
\hline \multicolumn{4}{|c|}{ b-Antimonene } & \multicolumn{4}{|c|}{ b-Antimonene } \\
\hline \multicolumn{4}{|c|}{$\mathrm{E}$} & \multicolumn{4}{|c|}{$\mathrm{E}$} \\
\hline Adatom & $\mathrm{a}$ & $\mathrm{m}$ & $\mathrm{P}$ & Adatom & $\mathrm{a}$ & $\mathrm{m}$ & $\mathrm{r}$ \\
\hline $\mathrm{H}$ & 1.25 & 1.0 & 0.297 & $\mathrm{Si}$ & 1.93 & 2.0 & 0.235 \\
\hline $\mathrm{Li}$ & 1.41 & 0.0 & 0.218 & $\mathrm{P}$ & 1.30 & 1.0 & 0.240 \\
\hline B & 4.04 & 1.0 & 1.235 & $\mathrm{Cl}$ & 2.26 & 0.0 & 0.630 \\
\hline $\mathrm{C}$ & 7.71 & 2.0 & 1.535 & $\mathrm{Ti}$ & 3.38 & 2.0 & 0.316 \\
\hline $\mathrm{N}$ & 1.87 & 0.0 & 1.309 & As & 1.22 & 1.0 & 0.481 \\
\hline $\mathrm{O}$ & 3.46 & 0.0 & 0.957 & In & 1.59 & 1.0 & 0.342 \\
\hline $\mathrm{Al}$ & 1.66 & 1.0 & 0.281 & $\mathrm{Sb}$ & 1.19 & 1.0 & 0.000 \\
\hline
\end{tabular}

Compared with the doping in phosphorene and arsenene, the doping of antimonene is in the initial stage of exploration. ${ }^{39,69,174,175}$ The geometric structures, stabilities, electronic and magnetic properties of buckled antimonene have been studied with substitutional doping of 3d-transition-metal adatoms, such as Sc, Ti, V, Cr, Mn, Fe, Co, Ni, Cu and Zn. ${ }^{174}$ They found that the $\mathrm{Cr}$ doping induces a spin-polarized semiconducting state, while half-metallic states appear in the $\mathrm{Ti}, \mathrm{V}$, and $\mathrm{Mn}$ doped antimonene. Ciraci et al. ${ }^{39}$ studied selective adatoms (H, Li, B, C, N, O, Al, In, Si, P, Cl, Ti, As and Sb) and molecules $\left(\mathrm{H}_{2}, \mathrm{O}_{2}\right.$, and $\mathrm{H}_{2} \mathrm{O}$ ) on buckled antimonene. Calculations indicated that all adatoms have a strong binding with antimonene, whereby its electronic structures are modified considerably. Specific adatoms (H, B, C, Al, $\mathrm{Si}, \mathrm{P}, \mathrm{Ti}, \mathrm{As}$, In and $\mathrm{Sb}$ ) lead to spin polarization for buckled antimonene. The energy locations of spinpolarized states for $\mathrm{C}, \mathrm{Si}$, and $\mathrm{Ti}$ atoms suggest that antimonene can attain half metallicity at high coverage of one of these adatoms. Xie et al. ${ }^{175}$ also introduced TTF and TCNQ as electron and hole dopants to attain n- and p-type antimonene semiconductors. After adsorption of TTF and TCNQ, the band gap of antimonene significantly decreases. 
Chemical functionalization has been proposed as a powerful way for manipulating quantum properties of 2D materials. Group-VA family, phosphorene, ${ }^{176-180}$ arsenene, ${ }^{46,53-55,59,181}$ antimonene ${ }^{63,137,182-184}$ and bismuthene ${ }^{185-189}$ have been further functionalized with $\mathrm{H}, \mathrm{F}, \mathrm{Cl}, \mathrm{Br}, \mathrm{I}, \mathrm{O}, \mathrm{OH}, \mathrm{CH}_{3}$ and other functional groups, which induce many novel structural, magnetic, electronic properties and topological edge states (Fig. 8c). Different from physical adsorption, chemical functionalization causes large areas of reconstruction of $2 \mathrm{D}$ materials.

Among functionalized group-VA 2D materials, phosphorene is the first one to be investigated. Wang et al. studied the physical and chemical properties of phosphorene oxides. ${ }^{176}$ Its phonon spectrum shows that phosphorene oxides are stable under ambient conditions. They found that the degree of Ofunctionalization of phosphorene can influence its band gap. Furthermore, the band gap of phosphorene oxide can be tuned effectively by external strain or an electric field. Lately, Zhu et al. proposed a new stable 2D blue phosphorene oxide (BPO). ${ }^{177}$ First-principles calculations indicate that BPO is found to be a narrow bandgap semiconductor and it can be subjected to a semiconductor-to-semimetal quantum phase transition under strain. Moreover, they pointed out exotic effects via building effective models characterizing the phase transition and novel emergent fermions. BPO will make contributions to explore fundamental properties of quantum phase transitions and fermions, and it can be a candidate for nanodevice applications.

Inspired by these fantastic properties of the functionalized phosphorene, researchers have already started to explore other group-VA 2D materials. Zhang et al. predicted a new planar magnet and 2D Dirac materials derived from the hydrogenated arsenene. ${ }^{46}$ They found that the semi-hydrogenated arsenene is a quasi-planar magnet. However, quite interestingly, the fully hydrogenated arsenene is an extremely planar Dirac material, with three-fold rotation symmetry like graphene. The electronic properties of arsenene are modulated by hydrogen, changing from a semiconductor (bare arsenene) to a zero-band gap metallic material (partially hydrogenated arsenene), and further to a Dirac material (fully hydrogenated arsenene). Subsequently, structural and electronic properties of arsenene functionalized by halogenation, hydroxylation and methylation are also reported. Unambiguously, the buckling height of pristine arsenene is greatly decreased by the hydrogenation, resulting in a planar sheet. Other polar group $(\mathrm{F}, \mathrm{Cl}, \mathrm{Br}, \mathrm{I}, \mathrm{OH}$ and $\mathrm{CH}_{3}$ ) functionalized arsenene monolayers have different buckling heights of $0.11,0.05,0.07,0.14$, 0.06 and $0.02 \AA$, respectively. This subtle structural difference of these functionalized arsenene structures has brought unprecedented richness in their electronic properties, such as various bulk gaps showing potential in quantum spin Hall insulator applications. ${ }^{54,55,59}$

Analogously, these polar groups have also been introduced into antimonene and bismuthene monolayers to tailor their structural and electronic properties. Noticeably, the spin-orbital coupling has opened a quantum spin Hall gap in chemically decorated arsenene, antimonene and bismuthene. ${ }^{63,185}$ The calculated edge states of nanoribbons further demonstrated that functionalized arsenene, antimonene and bismuthene are good 2D topological insulators. This aspect will be described in the following section. To sum up, chemical functionalization is an effective method to manipulate the geometry, electronic structures and topological properties of 2D group-VA nanosheets.

Heterostructures. Many studies have focused on van der Waals heterostructures (vdWHs) as shown in Fig. 8d. A typical vdWH is composed of two different monolayers free of dangling bonds, such as graphene/BN, graphene/TMDCs, graphene/phosphorene, BN/TMDCs, BN/phosphorene, TMDCs/ phosphorene, and TMDCs/MXenes. A great number of research studies have demonstrated that these vdWHs present many novel electronic and optical properties far beyond their individual components and can be regarded as a promising way to integrate the characteristics of their individual components with supreme properties to fabricate ideal electronic and optoelectronic devices. Therefore, two interesting questions arise: (1) What new properties of vdWHs can be measured based on 2D group-VA materials? (2) Will these new properties exceed pristine 2D group-VA crystals? As the novel 2D group-VA family 
members, phosphorene, ${ }^{190-206}$ arsenene $e^{207-211}$ and antimonene ${ }^{42,209,212}$ provide potential building blocks for vdWHs. In the phosphorene-based heterostructure, Guo et al. investigated the heterobilayers of phosphorene with a TMDC monolayer which possess properties of a semiconductor, and found that their bandgaps can be reduced by a vertical electric field. ${ }^{199}$ The power conversion efficiency for the phosphorene/ $\mathrm{MoS}_{2}$ heterobilayer can be up to $17.5 \%$. Besides, Wang et al. investigated a graphene/ phosphorene/graphene heterostructure. ${ }^{194}$ Based on their computational results, vdWHs have two advantages: (1) the thermionic transport barriers can be tuned by changing the number of layers; (2) thermal conductance across these non-covalent structures is weak. It is widely believed that layered van der Waals structures have potential to be a candidate for solid-state energy-conversion devices. Afterwards, Yu et al. examined the physical properties of a GeSe/phosphorene van der Waals $\mathrm{p}-\mathrm{n}$ heterostructure. ${ }^{205}$ They found that the heterostructure holds a type-II band alignment and indirect band gap. Through external strain, interesting indirect-direct and insulator-metal transitions can be achieved. Besides, they found spontaneous electron-hole charge separation, and thus, the GeSe/phosphorene heterostructure has the potential to be applied in optoelectronic devices.

In an arsenene-based heterostructure, Wang et al. and Xia et al. studied the structural and electronic properties of arsenene/graphene. ${ }^{209,210}$ They found that a linear Dirac-like dispersion relation is still retained in the arsenene/graphene heterostructure. Promisingly, by tuning the interlayer distance, the transition from a p-type Schottky barrier to an n-type Schottky barrier allows considerable freedom in integrating arsenene and graphene with controllable functions. However, for arsenene/silicene, the Schottky barriers are always p-type with a small band gap, which can be opened with a linear varying trend by changing the interlayer distance. By arsenene/ $\mathrm{FeCl}_{2}$ interfacial coupling, the spin splitting appears at the conduction band minimum of arsenene with a maximum splitting energy of $123 \mathrm{meV}$ in all arsenene/ $\mathrm{FeCl}_{2}$ vdWH configurations. In addition, antimonene/graphene, antimonene/ silicene and antimonene/germanene heterostructures have also been predicted. Antimonene/silience possesses a p-type Schottky barrier with a sizeable band gap opened at the Dirac point, and antimonene/graphene reveals a Shottky-to-Ohmic contact transition caused by compressive strain. In particular, for antimonene/germanene vdWHs, a significant band gap is opened with a direct band gap nature, differing from the indirect band gap feature of antimonene and gapless characteristic of germanene. Therefore, stacking vdWHs is an important and reliable method to tune the electronic properties of $2 \mathrm{D}$ group-VA crystals.

Unlike the well-known vertically stacked vdWHs, lateral heterostructures are connected in-plane by covalent bonds with atomically clean and sharp interfaces. This kind of hetero-structure has opened up various charming realms in materials science and device physics, marking the ultimate thickness limit for heterostructures. The 2D crystals from the group-VA layer materials, i.e. arsenene and antimonene, have been successfully used as building blocks to assemble such lateral heterostructures. ${ }^{42}$ After stitching As and $\mathrm{Sb}$ monolayers in-plane, the authors found that the freestanding $\mathrm{As} / \mathrm{Sb}$ lateral hetero-structures with the zig-zag interlines can present excellent stability, and possess intrinsic direct energy gaps without any modulations compared with the pristine As and Sb monolayers. Importantly, these lateral heterostructures linked by covalent bond tend to form a type-II band alignment, which could promote charge carrier separation spatially. Moreover, further studies revealed that the high charge carrier mobility is independent of the widths of the building blocks (arsenene and antimonene). The above qualities would make these materials potential candidates for applications in electronics and opto-electronics instead of the traditional heterostructures. In addition, they also identified the critical structural condition to achieve the highly coveted direct energy gaps. So, the fabrication of As/Sb lateral heterostructures will not only enrich the family of the new heterostructure with group-VA layer materials, but also highlight the efficient strategy to yield appealing materials for future devices. 


\section{Realizing 2D group-VA semiconductors}

\subsection{Fabrication of phosphorene}

Mechanical exfoliation of phosphorene. From a historical perspective, micromechanical exfoliation is the first method introduced to delaminate material down to the single layer morphology. The mechanical exfoliation technique has been broadly adopted in the preparation of black phosphorene, due to its simplicity and executability to produce high-quality 2D materials (Table 4). ${ }^{16,121,213-227}$ By means of a scotch tape, phosphorene is exfoliated from bulk crystals and then transferred onto the substrates inside a glove box. The most widely used substrate materials are $\mathrm{SiO}_{2} / \mathrm{Si}$, and other substrates are also implemented, such as $\mathrm{SiN}$ and glass. Phosphorenes having few-layer to monolayer thickness have been successfully obtained in recent experiments. However, these ultrathin phosphorenes peeled from the bulk crystals may suffer severe degeneration when being exposed to ambient conditions. Many approaches have been used to protect phosphorene from degradation and using various kinds of protection materials, including $\mathrm{AlO}_{\mathrm{X}}{ }^{222}$ PMMA (poly-methyl methacrylate), ${ }^{225}$ PMMA/PVA (poly vinyl alcohol), ${ }^{226}$ ZEP520A, ${ }^{227}$ PMMA/MMA (methyl methacrylate), ${ }^{214}$ and PDMS (polydimethylsiloxane). ${ }^{215-217,224}$ As illustrated in Fig. 9a, the phosphorene flakes exfoliated from bulk crystals are released onto the PMMA/PVA stack. Then the flake together with the PMMA/PVA is attached to the $200 \mathrm{~nm}$-thick freestanding SiN substrate film. To remove the PMMA/PVA layer, the whole sample is immersed in plenty of alcohol $(470 \mathrm{~mL})$ and acetone for a long soaking time $(412 \mathrm{~h})$ to remove the PMMA residue extensively and then finally dried with nitrogen. ${ }^{226}$

Conventional micromechanical exfoliation generally results in a low density of phosphorene flakes, leaving traces of tape on the surface and hence reducing the quality of phosphorene. Some investigations were also devoted to ameliorating the mechanical exfoliation technique. ${ }^{216-218,254,255}$ Castellanos-Gomez et al. employed an intermediate viscoelastic surface to exfoliate the flakes substantially, which increases the yield and reduces the contamination of the fabricated flakes. ${ }^{217}$ Another method to modify the mechanical exfoliation technique is to use two PDMS stamps. The first one is reticulated in a Petri dish while the second one is reticulated in a cylindrical tube of $1 \mathrm{~cm}$ diameter. After being exfoliated onto a flat PDMS stamp, the flake is transferred onto a curved PDMS stamp, instead of the desired substrate. ${ }^{216,254}$ Then, the curved PDMS stamp covered with flakes is rolled onto the substrate (Fig. 9b). These processes are always carried out under a dry atmosphere without any solution to remove the protection materials, which avoids the impurities left on the surface of flakes. To characterize the thickness of phosphorene flakes, AFM and Raman spectroscopy are the most convenient and simplest techniques. However, AFM and Raman spectroscopy are not reliable for characterizing monolayer or bilayer phosphorene. Due to the faster degradation rate of phosphorene, AFM cannot acquire enough data before significant sample degradation. Meanwhile, phosphorene can also be destroyed by the high-power laser used in Raman spectroscopy. Yang et al. reported a rapid, noninvasive and highly accurate approach to study the layer number of phosphorene, using phase-shifting interferometry (PSI). ${ }^{219}$ The results were further confirmed with reliable photo-luminescence measurements. PSI utilizes almost non-focused and very low-density light from a light-emitting diode source to achieve fast imaging to prevent any damage to the phosphorene samples. Fig. 9c and d show the optical microscope images of the monolayer and bilayer phosphorene on $\mathrm{Si} / \mathrm{SiO}_{2}$ substrates, respectively. 
(a)

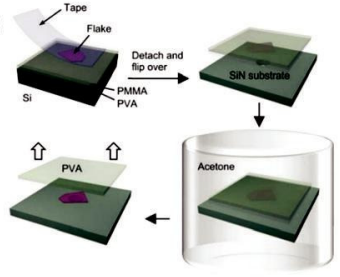

(c)

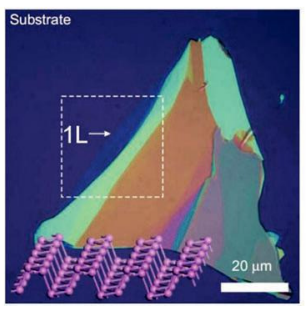

(b)

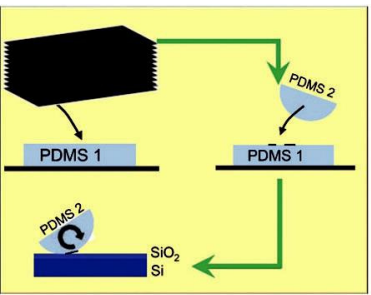

(d)

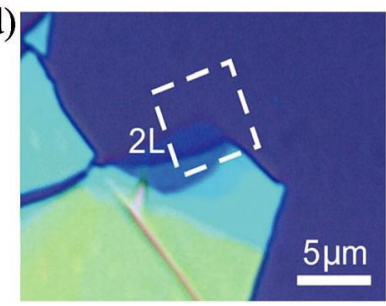

Fig. 9 (a) Sketch of the flake preparation and transfer process. Reproduced from ref. 226 with permission from Nature Publishing Group, copyright 2015. (b) Three-step exfoliation of black phosphorus with PDMS. (i) Exfoliation done on the flat PDMS-1, (ii) flakes are rolled on the semi-spherical PDMS-2 stamp and (iii) the stamp is rolled on the $\mathrm{SiO}_{2} / \mathrm{Si}$ substrate. Reproduced from ref. 216 with permission from Nature Publishing Group, copyright 2015. (c) Optical microscope image of a monolayer phosphorene (labeled as "1L"). The inset is the schematic of the single-layer phosphorene molecular structure. Reproduced from ref. 219 with permission from Nature Publishing Group, copyright 2015. (d) Microscope image of the phosphorene bilayer. Reproduced from ref. 220 with permission from the American Chemical Society, copyright 2014.

Table 4 Summary of synthesis methods used to prepare 2D group-VA materials

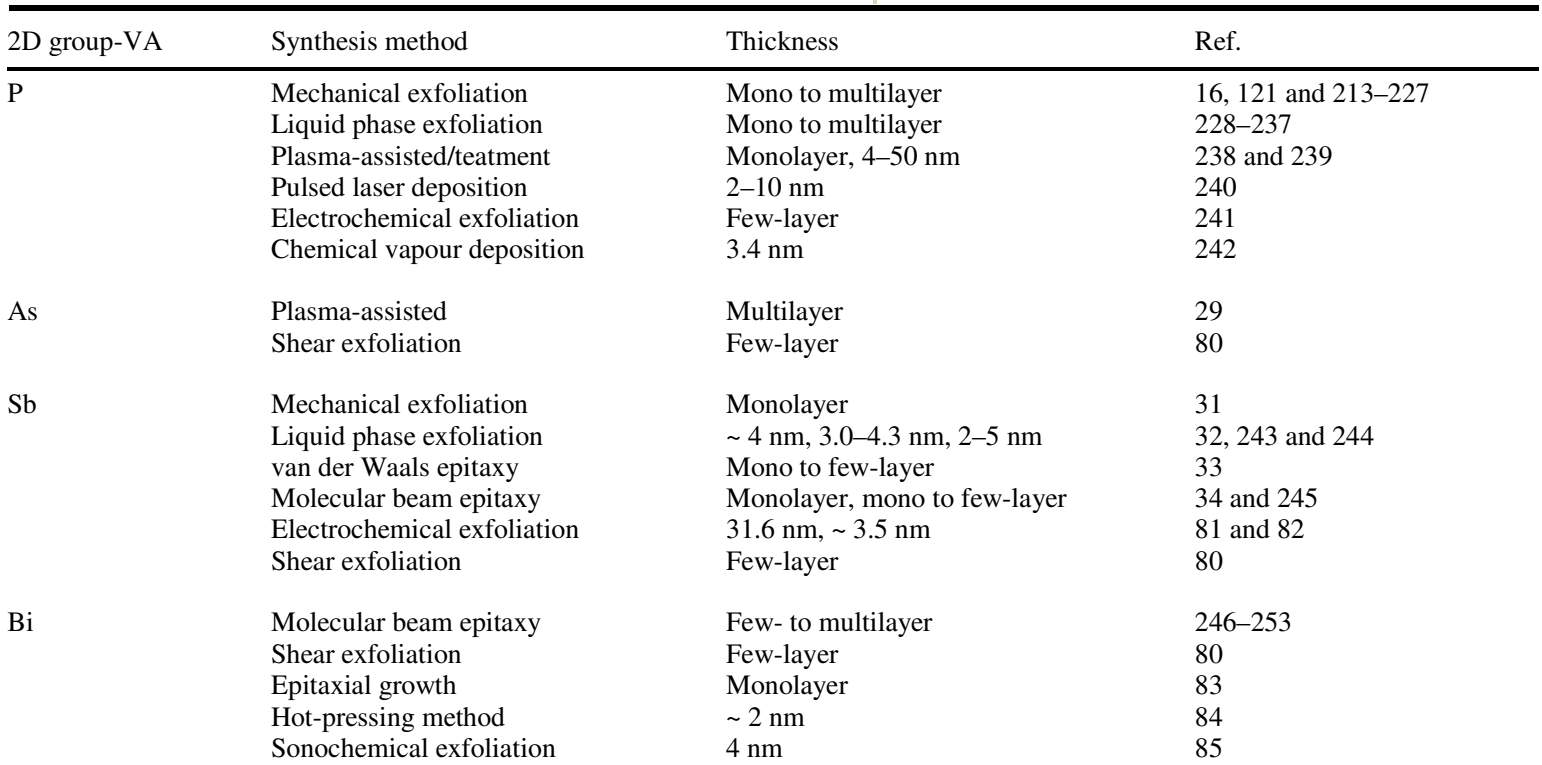

Liquid phase exfoliation of phosphorene. Although phosphorene produced by mechanical exfoliation has high structural and electronic quality, the yield is so low that it cannot meet the industrial requirements. Meanwhile, liquid phase exfoliation (LPE) has been applied to produce 2D materials and many efforts are made to get high-yield production of phosphorene (Fig. 10a-e). In the LPE, the exfoliation depends on a surface energy match between the solvent and the $2 \mathrm{D}$ material. It is important to 
choose a suitable solution to achieve efficient exfoliation and create a stable dispersion. Common liquid exfoliation solvents are listed in Fig. 10b. The LPE technique is almost based on ultrasonication, including organic solvent-based exfoliation, stabilizer-based exfoliation, ionic liquid-based exfoliation, salt-assisted exfoliation, intercalant-assisted exfoliation and ion exchange-based exfoliation. In addition, ultra-sonication can be performed either by the bath sonication or the tip sonication technique.

Organic solvents have been broadly applied to exfoliate black phosphorene. ${ }^{228,229}$ In 2014, Brent et al. firstly reported the liquid exfoliation of black phosphorus in N-methyl-2-pyrrolidone (NMP) to fabricate few-layer phosphorene. ${ }^{228}$ Afterwards, researchers were committed to modify the LPE method to obtain a highly-concentrated and uniform solution, in order to produce large-scale and stable phosphorene. Guo et al. added $\mathrm{NaOH}$ to NMP solvent, called the basic-NMP solvent, and produced phosphorene with excellent water stability, controllable size and layer number, as well as in high yield. ${ }^{229}$ After 4 hour sonication, the phosphorene in basic-NMP was separated and transferred to water by centrifugation. It is interesting that the basic-NMP can realize more efficient phosphorene exfoliation and excellent stability than pure NMP and water. This is because $\mathrm{OH}$-ions are adsorbed on the surface of phosphorene, which leads to a negative charge and a suspension with a zeta potential over $30 \mathrm{mV}$. They also found that optimizing the centrifugation speed can produce phosphorene with a relatively uniform size and thickness. For instance, centrifugation at $18000 \mathrm{rpm}$ can generate phosphorene with a size of around 210 $\mathrm{nm}$ and a thickness of $2.81 .5 \mathrm{~nm}$ (2-7 layers) and a thickness of $5.32 .0 \mathrm{~nm}$ (5-12 layers). Yasaei et al. examined several solvents from different chemical families for black phosphorus exfoliation. ${ }^{230}$ They noticed that dimethyl-formamide (DMF) and dimethyl sulfoxide (DMSO) are another two appropriate solvents to produce uniform and stable dispersions. Hanlon et al. demonstrated that phosphorus nanosheets produced by liquid phase exfoliation under ambient conditions in solvents such as $\mathrm{N}$ cyclohexyl-2-pyrrolidone (CHP) showed high-quality, controllable size, large quantities and observable photoluminescence. ${ }^{231}$ They exfoliated black phosphorus in an aqueous surfactant solution with sodium cholate $(\mathrm{NaC})$ as the stabilizer. Compared with mechanically cleaved flakes, phosphorene exfoliated by this method is extremely stable in CHP, probably because of the solvation shell which can protect the nanosheets from reacting with water or oxygen. 


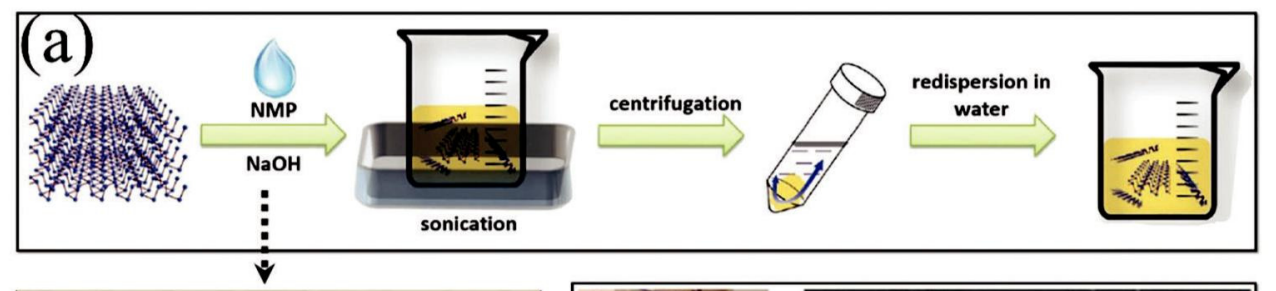

(b) Common Solvents

NMP DMF CHP

IPA DMSO EA

[BMIM] [TFO] [BF4]

Water Ethanol PBS $\cdots$
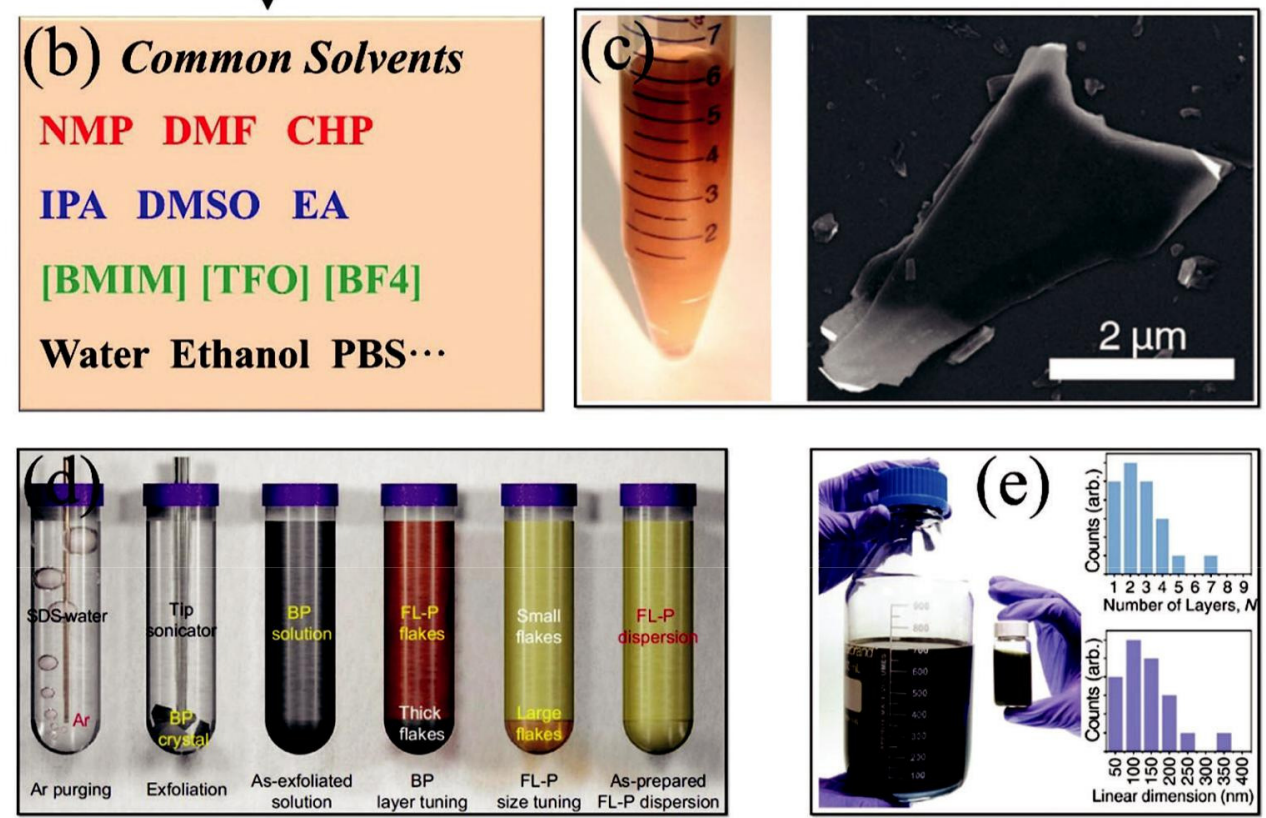

Fig. 10 (a) Schematic illustration of the fabrication process of basic-NMP-exfoliated phosphorene. Reproduced from ref. 229 with permission from Wiley, copyright 2015. (b) Common liquid exfoliation solvents. (c) Photograph of a liquid-exfoliated suspension of 2D phosphorene in isopropyl alcohol, and its SEM image. Reproduced from ref. 237 with permission from the American Chemical Society, copyright 2015. (d) Schematic of the preparation method for few-layer phosphorene aqueous dispersions. Reproduced from ref. 234 with permission from the National Academy of Sciences, copyright 2016. (e) Scaled-up production of 2D phosphorus, using a combination of shear mixing and sonication. The size distribution of $2 \mathrm{D}$ phosphorus in this fraction is shown (right). Reproduced from ref. 237 with permission from the American Chemical Society, copyright 2015.

Ionic liquids present a stable yield with a high concentration for efficient exfoliation of 2D materials. Zhao et al. used nine ionic liquids to produce stable monolayer to few-layer black phosphorene, which is produced on a large-scale, is highly concentrated and environmentally friendly. ${ }^{233}$ The prepared dispersions remained stable for one month without any obvious sedimentation and aggregation in ambient air. Kang et al. presented the tip-sonication-based exfoliation method to produce phosphorene at high power output in an inert environment. ${ }^{232,234}$ Tip-sonication-based exfoliation has the distinguishing feature that it uses a sealed container lid attached to an ultrasonicator tip/probe. The interface between the tip and the lid is sealed with PDMS several times to occlude $\mathrm{O}_{2}$ and $\mathrm{H}_{2} \mathrm{O}$ penetration into the tube. In addition, Parafilm ${ }^{\mathrm{s}}$ and Teflon ${ }^{\mathrm{s}}$ tapes are used to further suppress ambient exposure. Compared with bath sonication, tip sonication is driven at a higher power, immensely saving the processing time. Instead of conventional organic solvents with high boiling points, ${ }^{235}$ Kang et al. ${ }^{234}$ used deoxygenated water with $2 \%(\mathrm{wt} / \mathrm{vol}$ ) sodium dodecylsulfate as the surfactant for preparing phosphorene (Fig. 10d). Deoxygenated water is prepared by purging deionized water with ultrahigh-purity Ar gas in a sealed container. For fewlayer phosphorene enrichment, as-prepared solutions undergo two steps of sedimentation-based ultracentrifugation to isolate thin flakes with a relatively large lateral area. Exfoliated phosphorus nanosheets are stabilized in aqueous solution with amphiphilic surfactants in a manner analogous to other 
2D materials with higher optical absorbance and a lower zeta potential value. This method produced stable and highly concentrated few-layer phosphorene with distinct advantages over the ones exfoliated in organic solvents. Moreover, research studies have shown that the LPE method can exfoliate phosphorene in large yield assisted by a shear mixing process which applies the shear force to break down the interlayer van der Waals forces, and this can also reduce the ultrasonication time. ${ }^{236,237}$ Fig. 10c and e demonstrate the presence of phosphorus nanosheets with lateral dimensions in micrometer sized and scaled-up production of 2D phosphorus, respectively.

Other fabrications of phosphorene. Other approaches to fabricate few-layer phosphorene are also utilized, such as plasma-assisted fabrication, ${ }^{238,239}$ pulsed laser deposition, ${ }^{240}$ electrochemical exfoliation, ${ }^{241}$ and chemical vapour deposition. ${ }^{242}$ As an emerging approach, 2D monolayers can be obtained from multilayered 2D materials via plasma or laser irradiation. ${ }^{256,257} \mathrm{Lu}$ et al. proposed a successful experimental method to fabricate monolayer phosphorene through mechanical cleavage and a subsequent $\mathrm{Ar}^{+}$plasma thinning process. ${ }^{238}$ This method is strongly controllable for fabrication of fewlayer phosphorene. Moreover, Jia et al. reported the preparation of thickness-controlled few-layer black phosphorus films by means of the modulated plasma treatment of phosphorus flakes. ${ }^{239}$ By regulating the plasma treatment duration, black phosphorus thickness can be effectively controlled. Another method to obtain few-layer black phosphorus is pulsed laser deposition. Yang et al. provide a new conventional and scalable technique, pulsed laser deposition, which can successfully deposit a wafer scale ultrathin film at relatively low temperature. ${ }^{240}$

\subsection{Fabrication of arsenene}

The synthesis of monolayer buckled arsenene has not been reported to date. Tsai et al. demonstrated a new kind of preparation technique, the plasma-assisted process, to synthesize multilayer arsenene on InAs, as shown in Fig. $11 \mathrm{a} .{ }^{29}$ During the multilayer arsenene fabrication process, they chose to launch nitrogen ions into the InAs substrate. During the thermal treatment, the $\mathrm{N}$ ions react with In, and As is squeezed out of the surface to form the arsenene layers. To optimize the synthesis process of arsenene, the annealing time, power of plasma and exposure time of plasma must be well controlled. After a systematic investigation, they found that the optimized synthesis process would begin with the $\mathrm{N}_{2}$ plasma immersion with a power of $100 \mathrm{~W}$ for $30 \mathrm{~min}$ and then annealing at $450 \mathrm{1C}$ for $30 \mathrm{~min}$. It is noted that the synthesized multilayer arsenene is not continuous but like a pile of multilayer nanoribbons. Besides, the method has also been adopted to synthesize multilayer graphene, germanene, violet phosphorene and silicene on certain substrates. ${ }^{258-261}$ Zhao et al. theoretically predicted a way to exfoliate buckled arsenene into single- or few-layers by the conformational change associated with the photo-isomerization of azobenzene (AB)-based molecules. ${ }^{60}$ The same photochromic system has been successfully used to exfoliate graphite to graphene during the liquid-phase exfoliation process. ${ }^{262}$ As demonstrated in Fig. $11 \mathrm{~b}$, when exposed to ultraviolet and visible light, the trans-to-cis conformational change of $\mathrm{AB}$ or $\mathrm{AB}$ $\mathrm{OC}_{10} \mathrm{H}_{21}$ can lead to an increase of the interlayer distance. Reactive molecular dynamics simulations show that about $5 \AA$ increase of interlayer distance can be obtained during the photo-isomerization of an $\mathrm{AB}$ $\mathrm{OC}_{10} \mathrm{H}_{21}$ molecule, nearly $4 \AA$ larger than that induced in the $\mathrm{AB}-\mathrm{OC}_{10} \mathrm{H}_{21}$-graphene hybrid system. ${ }^{262}$ These results indicate the possibility of the effective exfoliation of multilayer arsenene by AB-based molecules. The predicted results may pave the way for the experimental realization of light-driven synthesis of single or few-layer arsenene.

By the aqueous shear exfoliation method, Gusmao et al. successfully fabricated $\mathrm{As}, \mathrm{Sb}$ and $\mathrm{Bi}$ nanosheets. ${ }^{80}$ Here, we mainly introduce the experimental procedure for As nanosheets, which is similar to those for $\mathrm{Sb}$ and Bi. First, to purify the powders, As bulk should be pretreated to remove the oxides on 
the surface. $1 \mathrm{~g}$ of As powder with $5 \mathrm{mg} \mathrm{mL}^{1}$ aqueous surfactant sodium cholate is sonicated in an ice bath for 20 minutes. After being centrifuged with a Beckman Coulter Allegra 64R centrifuge for 1 hour at $4 \mathrm{k} \mathrm{rpm}$, the sediment is then dried at a temperature of $601 \mathrm{C}$ in a vacuum. Then, the pretreated As powders are mixed in aqueous sodium cholate surfactant for shear dispersion and exfoliation. The exfoliation process continues for 2 hours alternating between two types of kitchen blenders, a bottom shear mixer and a top shear mixer, as shown in Fig. 11c. The As nanosheets are separated by centrifugation with aqueous washing and centrifugation at different rotation speeds. Finally, the sediments are dried at $601 \mathrm{C}$ in a vacuum. They investigated the structure, morphology, and composition of exfoliated As nanosheets by TEM and EDS (Fig. 11d). The individual particles of As nanosheets are clear in sizes of tenths of nanometers. They also measured Raman spectra to study the crystal structure and thickness and educed that the exfoliated materials have nanoscale thickness.

(a)

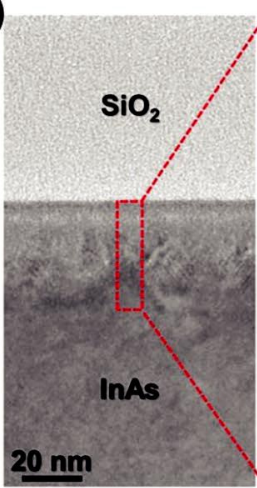

(c)

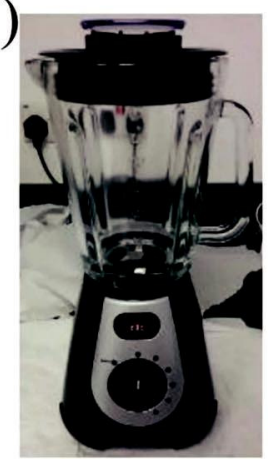

Bottom shear mixer
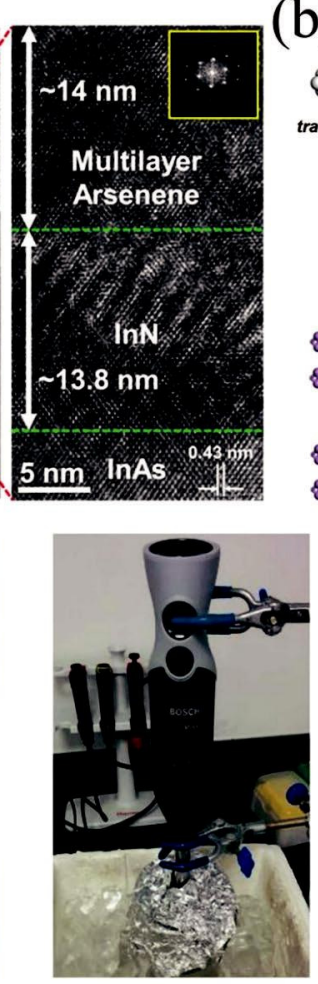

Top shear mixer (b)

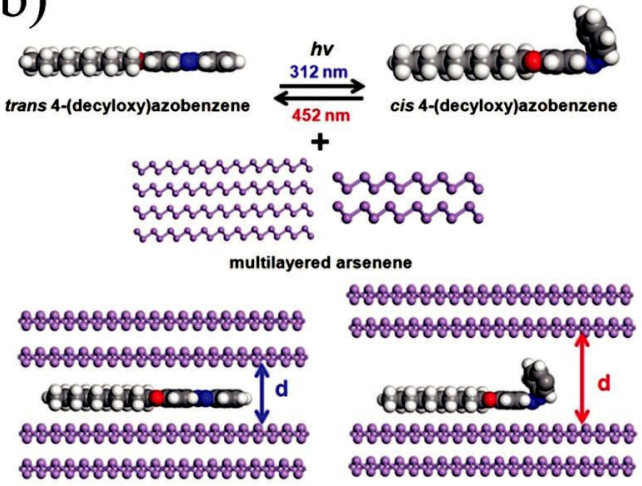

(d)

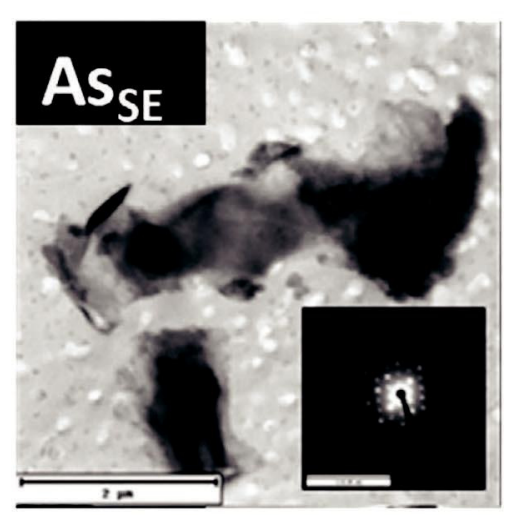

Fig. 11 (a) TEM image of multilayer arsenene/InN/InAs. Reproduced from ref. 29 with permission from the American Chemical Society, copyright 2016. (b) The predicted light-assisted exfoliation of multilayer arsenene by the photo-isomerization of 4(decyloxy)azobenzene $\left(\mathrm{AB}-\mathrm{OC}_{10} \mathrm{H}_{21}\right)$. Reproduced from ref. 60 with permission from the Royal Society of Chemistry, copyright 2017. (c) Bottom shear mixing and top shear mixing. (d) The TEM image of shear-exfoliated As. The scale bar corresponds to 2 mm. (c and d) Reproduced from ref. 80 with permission from Wiley, copyright 2017.

In addition, hydrothermal synthesis, as well as solvothermal synthesis, is a typical wet-chemical synthesis method to fabricate nanomaterials. It is a simple and scalable method to synthesize 2D materials in high yield and low cost. Some works about the hydrothermal/solvothermal synthesis of 2D materials have been reported. ${ }^{263-265}$ For group-VA nanosheets, nearly no work on their hydrothermal/solvothermal synthesis has been reported to date. However, in 1968, As bulk crystals were grown by the hydro-thermal synthesis approach using hydroiodic acid as a solvent. ${ }^{266}$ In addition, several works about the 
hydrothermal synthesis of quantum dots for $\mathrm{P},{ }^{267}$ nanotubes, nanowires and nanospheres for $\mathrm{Sb}$ and $\mathrm{Bi}$ have been reported. ${ }^{268-273}$ It is believable that through selecting suitable precursors and solvents at optimized temperature, As nanosheets and other 2D group-VA materials can be synthesized successfully in the near future.

\subsection{Fabrication of antimonene}

Two antimony allotropes are known, one is the most stable form, a rhombohedral structure (b-phase), similar to that of grey arsenic. Another one has an orthorhombic structure, known as the a-phase, which is similar to that of black phosphorus. As is stated above in Section 2.1, theoretical calculations predict that both a- and b-phase antimonene are energetically and structurally stable, indicated by their formation energies (in Fig. 3b) and phonon band dispersions (in Fig. 4b), repectively. ${ }^{27}$

Micromechanical exfoliation of antimonene. Ares et al. used the scotch tape method finding a low transfer yield of thick flakes and a very small amount of few-layer flakes. ${ }^{31}$ The yield can be improved by replacing the tape by a viscoelastic polymer (Gelfilm from Gelpak ${ }^{\mathrm{s}}$ ). The softness of the viscoelastic polymer results in a higher yield of flakes on the polymer surface. Then, by pressing the polymer against a $\mathrm{SiO}_{2}$ substrate, they were able to obtain thin antimony flakes with large area in a more controlled way.

Fig. 12a is an AFM image showing a few-layer antimonene flake with a well-defined monolayer terrace. Similar to the exfoliated graphene on $\mathrm{SiO}_{2}$, ripples can be formed by conformation of antimonene with respect to the underlying $\mathrm{SiO}_{2}$ substrate. The measured height of this terrace is $\sim 0.9 \mathrm{~nm}$ (Fig. 12a and b) and compatible with the presence of water layers between the flakes and $\mathrm{SiO}_{2}$ as is commonly observed in other 2D materials such as graphene or dichalcogenides. ${ }^{274,275}$ Raman spectroscopy is widely used in determining the layer thickness of many 2D materials. Unfortunately, the Raman signal measured for few-layer antimony flakes on silicon dioxide is so weak that it hampered the final determination of the flake thickness. The final determination of the layer height is carried out by AFM assisted nanomanipulation. ${ }^{31}$ According to Geim and Novoselov, ${ }^{275}$ the identification of single graphene sheets can be unambiguously carried out by measuring the step height of single folds. In this case, the lowest step height is $\sim 4 \AA$ (inset in Fig. 12c and profile in Fig. 12d) which corresponds to a single layer of bphase antimonene. XEDS and HRTEM analyses confirmed the high quality of the material and its rhombohedral structure (b-phase).

In order to demonstrate the stability of the flakes under ambient conditions, a region with two thin flakes was located (Fig. 12e and f). The sample was then stored for two months without further precaution. After that, the flakes were imaged again showing no significant evolution (Fig. 12e and g). To further confirm the resistance to moisture, an AFM image was acquired with the sample immersed in water (Fig. 12e and h). The surface retains the original morphology and the hexagonal periodicity (inset Fig. 12h) compatible with the b-antimony phase can be clearly visible. Fig. 12e is the corresponding line profiles of the AFM images taken under different conditions.

A simple and fairly accurate identification of the thicknesses of thin antimony flakes based on Fresnel-law has been reported. ${ }^{35}$ To this end, mechanically exfoliated antimony flakes were transferred to a $\mathrm{Si}(111)$ substrate with $300 \mathrm{~nm} \mathrm{SiO}_{2}$ grown on top. ${ }^{31}$ Fig. 13a shows a high-resolution optical microscopy image of a representative set of flakes illuminated with white light. Fig. 13b-e demonstrate the same flakes but illuminated with different light of selected wavelengths. In Fig. 13f, the optical contrast is colour coded as a function of the incoming light wavelength and the $\mathrm{SiO}_{2}$ thickness. It can be concluded that the highest optical contrast can be obtained by using light of wavelengths below $550 \mathrm{~nm}$ for substrates with 80 and $\sim 240 \mathrm{~nm} \mathrm{SiO}$ 2 thicknesses. This method allows a fast and fairly easy determination of the layer thicknesses that is particularly relevant since Raman spectroscopy fails to provide this information on $\mathrm{SiO}_{2}$ substrates. In summary, the works by Ares et al. demonstrate the 
feasibility of isolating and identifying high quality antimonene crystals which are very stable under ambient conditions. ${ }^{35}$
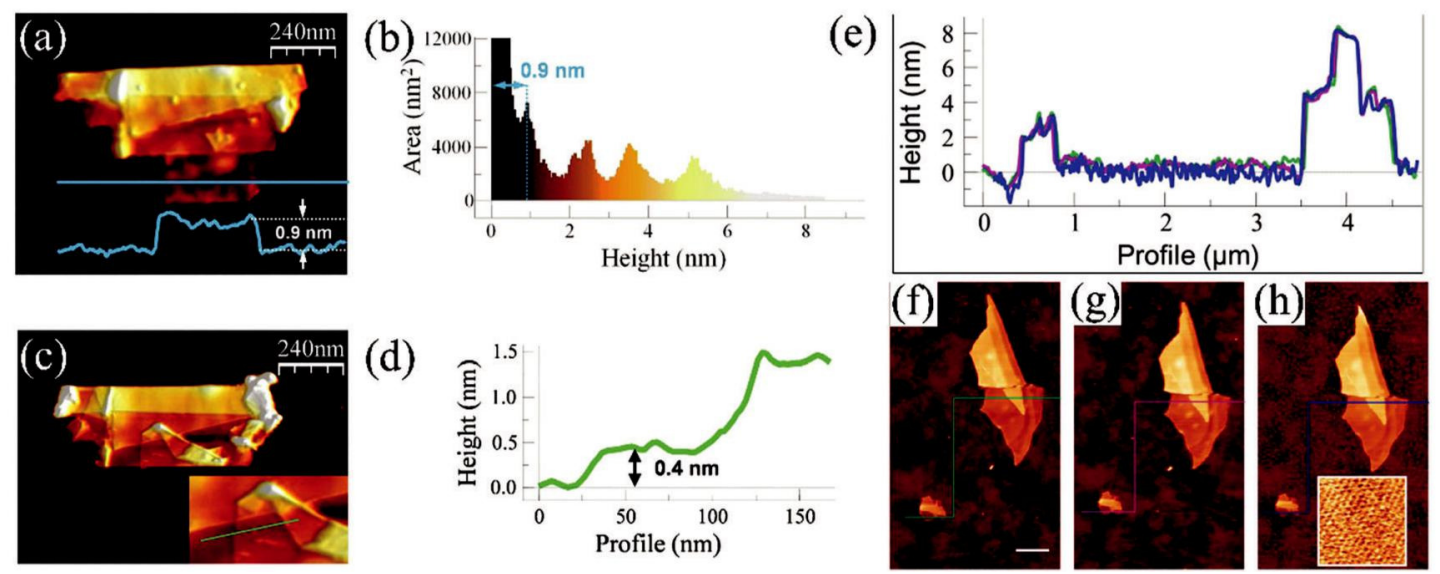

(d)
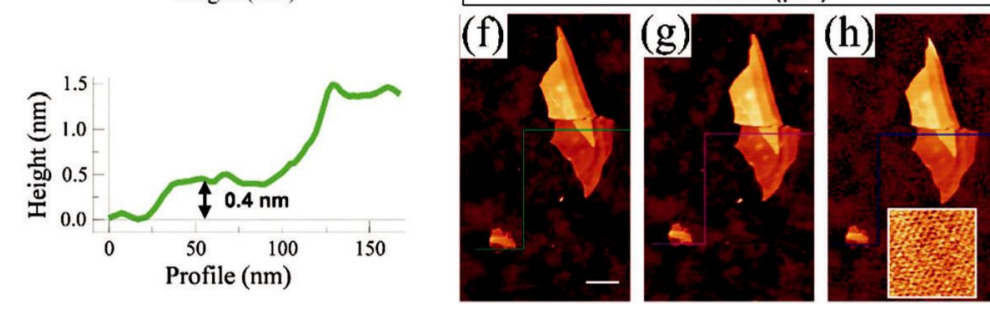

Fig. 12 AFM topography images of an antimonene flake with a monolayer terrace at the bottom. (a) AFM topography showing a $0.2 \mathrm{~mm}^{2}$ antimonene flake with terraces of different heights. The profile is taken along the blue horizontal line in the image. (b) Height histogram of the image in (a) where the different thicknesses of the terraces can be readily seen. For the sake of clarity, the substrate peak has been cut to $12000 \mathrm{~nm}^{2}$. The minimum step height is $\sim 0.9 \mathrm{~nm}$ compatible with a single layer of antimony adsorbed in the presence of water layers. (c) Same flake as in (a) but after a nanomanipulation process. The lower terrace of the flake was folded upwards resulting in an origami structure with different folds. The inset corresponds to the area of the origami where the lowest step height is found. (d) Profile along the green line in the inset in (c). The lowest step height is $\sim 0.4 \mathrm{~nm}$ corresponding to a single antimonene layer. (e) Profiles taken along the lines drawn in (f-h). (f) Image of two antimonene sheets taken immediately after exfoliation. White scale bar: $500 \mathrm{~nm}$. (g) Same as in (f) but measured two months later. (h) Image taken immediately after $(\mathrm{g})$ but with the sample immersed in water. The inset shows atomic periodicity compatible with the antimonene atomic lattice. Reproduced from ref. 31 with permission from Wiley, copyright 2016. 

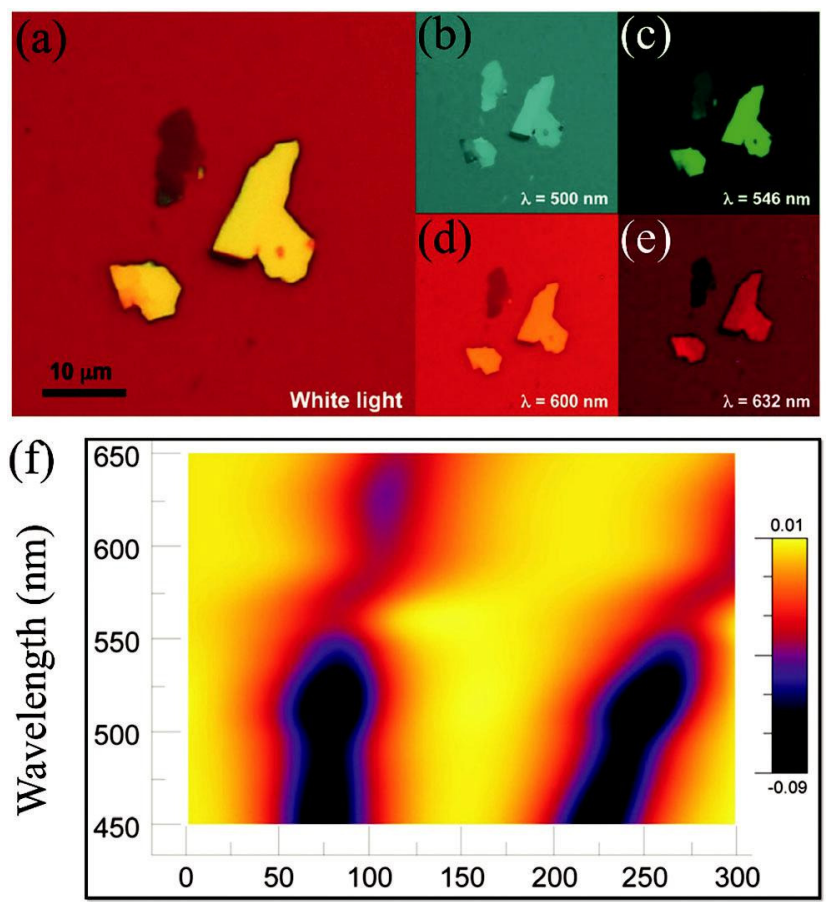

Fig. 13 Different optical images of few-layer antimonene flakes acquired using (a) white light and (b-e) narrow-bandpass filters $\left(500,546,600\right.$ and $632 \mathrm{~nm}$, respectively). (f) Optical contrast as a function of incident light wavelength and $\mathrm{SiO}_{2}$ layer thickness for antimonene. Reproduced from ref. 35 with permission from the American Chemical Society, copyright 2017.

Liquid phase exfoliation of antimonene. Liquid phase exfoliation has been successfully applied to acquire single- or few-layer of several 2D-materials on the large scale, ${ }^{240,241}$ including stable suspensions of few-layer black phosphorus. ${ }^{221}$ This is a powerful approach for the production of massive quantities of layers suspended in a variety of solvents and, which in principle is promising to be scalable for mass production. Gibaja et al. demonstrated that high-quality, few-layer antimonene nanosheets could be produced by liquid phase exfoliation. ${ }^{32}$ The sonication of bulk antimony crystals in an isopropanol ( $\left.{ }^{\mathrm{i}} \mathrm{PrOH}\right)$-water $(4: 1)$ mixture gives rise to a colourless and very stable dispersion showing the FaradayTyndall effect (Fig. 14a). The unexfoliated material is removed by centrifugation at $3000 \mathrm{rpm}$ (845 rcf) for 3 min to yield a stable dispersion with a concentration of ca. $1.7410^{3} \mathrm{~g} \mathrm{~L}^{-1}$ as determined by atomic absorption spectrometry. The liquid phase exfoliation parameters were optimized from independent studies of solvent selection, sonication experimental conditions and centrifugation speeds. The stability of the resulting suspensions was determined by UV-vis spectroscopy. Fig. 14b shows a typical AFM topographic image of few-layer antimonene flakes on $\mathrm{SiO}_{2}$ substrates. Fig. 14c displays the height histogram corresponding to the image shown in Fig. 14b. The flakes do not show characteristic terraces distinguishing layered materials but well-defined structures with heights being multiples of ca. $4 \mathrm{~nm}$. These features suggest that the apparent mono/bilayer thickness could be $4 \mathrm{~nm}$. It is well-known that apparent AFM heights of layers obtained by LPE can be overestimated due to residual solvent ${ }^{231,276}$ as well as contributions from effects such as capillary and adhesion forces. ${ }^{277}$ The overall lateral dimensions of the isolated nanolayers are over 1-3 $\mathrm{mm}^{2}$. High-resolution AFM topographic and Fast Fourier transform images taken in the lowest terrace (ca. $4 \mathrm{~nm}$ ) of the isolated flake shown in Fig. 14d and e exhibit an atomic periodicity indicating b-phase antimony. Aberration corrected scanning transmission electron microscopy (STEM) combined with electron energy-loss spectroscopy (EELS) was used to 
determine the structure and composition of the flakes. Fig. $14 \mathrm{f}$ exhibits an atomic resolution image of the crystal structure, obtained at an acceleration voltage of $80 \mathrm{kV}$ to prevent beam induced damage.

Remarkably, AFM studies of the on-surface isolated few-layer antimonene confirm that they are very stable for weeks under ambient conditions. In order to complete the structural characterization of the isolated few-layer antimonene flakes on $\mathrm{SiO}_{2}$ substrates, Raman spectroscopy is also utilized. The Raman spectrum of bulk antimony shows two main phonon peaks: $A_{1 g}$ at $150 \mathrm{~cm}^{1}$ and $E_{g}$ at $110 \mathrm{~cm}^{1}$, when excited off resonance (e.g. $1=532 \mathrm{~nm}){ }^{278}$ Studies on a polydisperse sample of antimony by correlation between AFM and Statistical Raman Microscopy (SRM) showed that flakes with an apparent thickness below ca. $70 \mathrm{~nm}$ hardly display any measurable Raman signal. This unexpected dependence of Raman intensities on flake thickness is similar to that of the micromechanically-exfoliated flakes of antimony. ${ }^{31}$ Indeed, a series of single point spectra measured at different positions confirmed a concomitant decrease of the peak intensity with the thickness. Theoretical phonon spectra calculations, carried out to understand these experimental observations, indicate two interesting behaviours. Firstly, a significant contraction of the in-plane lattice constant of few-layer antimonene compared to the bulk material when the thickness of the layers is decreased to a trilayer, bilayer and monolayer. As a result, the frequency of the bulk $\mathrm{E}_{\mathrm{g}}$ mode gradually increases from 88 to $167 \mathrm{~cm}^{1}$ in the monolayer. Secondly, the calculations suggest a strong influence of the thickness on the simulated Raman activity, which decreases by more than a factor of 1000 from bulk to monolayer Sb, which explains the experimentally observed absence of Raman signals in the thinnest flakes. In summary, the work by Gibaja et al. provides an industrially scalable and environmentally friendly approach for isolating few-layer antimonene in aqueous solution that can be of interest for the development of new antimonene-based technologies. ${ }^{32}$
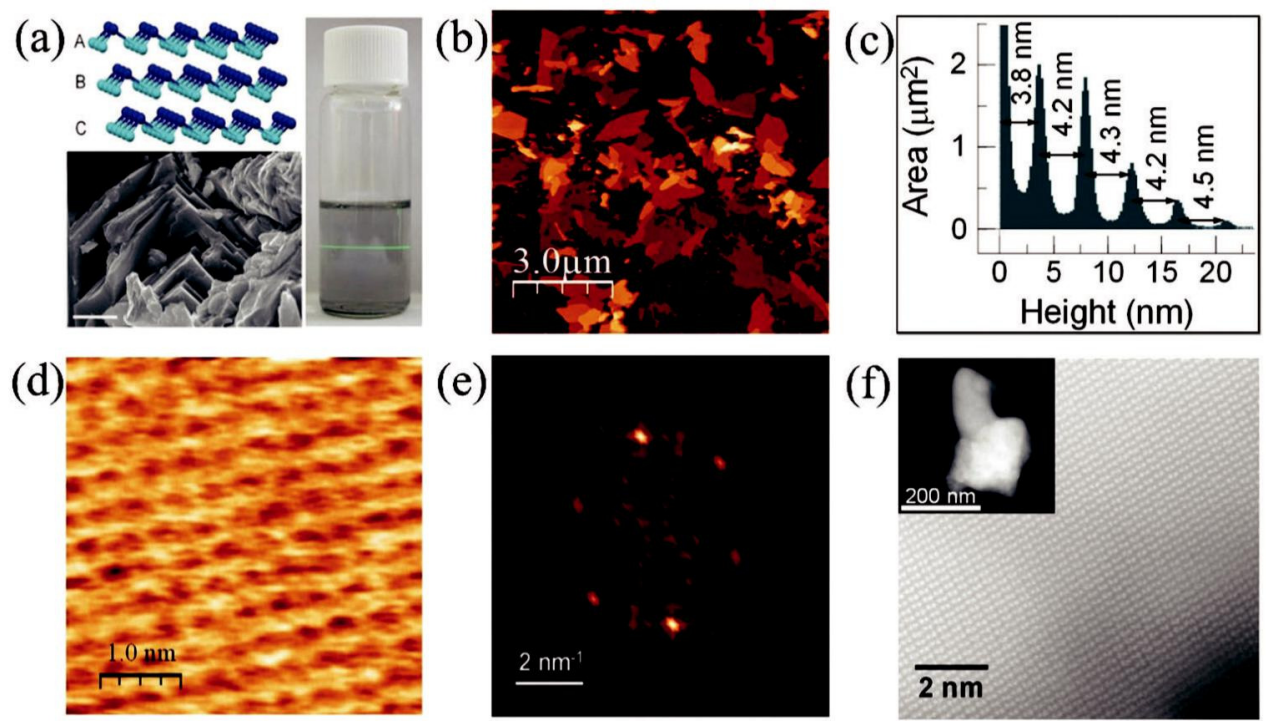

Fig. 14 (a) Structure of b-antimonene and SEM image of a layered antimony crystal (scale bar: $1 \mathrm{~mm}$ ) (left). Photograph of a dispersion of exfoliated FL-antimonene showing the Faraday-Tyndall effect. (b) Topographic AFM image of few-layer antimonene drop-cast on $\mathrm{SiO}_{2}$ showing over micron-scale lateral dimensions (scale bar, $3 \mathrm{~mm}$ ). (c) Height histogram of the image in (b) where the different thicknesses of the terraces can be readily seen. For the sake of clarity, the substrate peak has been cut to $2.5 \mathrm{~mm}^{2}$. A minimum constant thickness of ca. $4 \mathrm{~nm}$ can be easily observed. (d) AFM showing atomic periodicity. (e) Fast Fourier transform (FFT) image taken from (d), showing the agreement with a hexagonal lattice as expected for b-antimony. (f) Low magnification HAADF image of a flake along with an atomic resolution image (image taken along the [0 12 2] direction). Reproduced from ref. 32 with permission from Wiley, copyright 2016. 
van der Waals epitaxy of antimonene. van der Waals epitaxy has been successfully applied to grow various high quality layered materials. This technique uses conventional epitaxy growth of a material on a substrate that hampers dangling bond formation on its surface. Therefore, epitaxial layers are connected with the substrate via weak van der Waals forces instead of strong chemical bonding. Ji et al. have been able to grow high-quality few-layer antimonene monocrystalline polygons of the most stable b-phase antimony allotrope on a variety of substrates, including flexible ones (Fig. 15a-h). ${ }^{33}$ The experimental procedure (Fig. 15a) implements a two-zone tube furnace with separate temperature controllers in which antimony powder is placed in the $\mathrm{T} 1$ zone and heated up to $6601 \mathrm{C}$ to generate antimony vapour. The substrates are placed in the $\mathrm{T} 2$ zone of the furnace with a typical temperature of $3801 \mathrm{C}$ that produces antimony condensation. A transporting flow of $\mathrm{Ar} / \mathrm{H}_{2}$ gas was used to transfer antimony vapour from the $\mathrm{T} 1$ to the T2 area causing a soft-landing and diffusion of $\mathrm{Sb}$ atoms on the substrate and then allowing crystallization. After $60 \mathrm{~min}$ the temperature of the furnace was slowly cooled down up to room temperature. Inspection by optical microscopy confirms the formation of several polygonal shapes, including triangles, hexagons, rhombus and trapezoids (Fig. 15c-f). The crystal growth on mica can be split into nucleation and lateral growth (Fig. 15b). At the first stage, antimony vapours, carried by $\mathrm{Ar} / \mathrm{H}_{2}$ gas, cool down and land on the mica substrate, resulting in the formation of nuclei. Then, adatoms on mica diffuse to the edge of initial nuclei, which grow fast along the chemically passivated surface into layers. In particular, mica was found to be a very suitable substrate for van der Waals epitaxial growth of few-layer antimony due to the absence of dangling bonds on the ultra-smooth surface. The growth using silicon and sapphire substrates showed more irregularities. Therefore, the absence of dangling bonds seems to be crucial for the successful growth, which is confirmed by XPS. Most polygons have lateral sizes around 5-10 mm. AFM confirms a thickness down to $4 \mathrm{~nm}$ (ca. 10 atomic layers, Fig. 15g). A very small and irregular sheet with lateral size around $100 \mathrm{~nm}$ and thickness down to $1 \mathrm{~nm}$ (Fig. 15h), which is characteristic of monolayer antimonene, ${ }^{31}$ was also found. The b-phase formation was also confirmed by Raman spectroscopy. Interestingly, the Raman features observed for the single-crystalline antimonene layers show the intensity dependence reported for few-layer antimonene obtained by micromechanical exfoliation and LPE. ${ }^{31,32}$ However, signal detection of the Raman peaks of $\mathrm{E}_{\mathrm{g}}$ and $\mathrm{A}_{1 \mathrm{~g}}$ on mica is significantly enhanced in comparison to that observed on $\mathrm{SiO}_{2}$. HRTEM and SAED analyses confirmed the crystallinity and high quality of the material as well as corroborate its rhombohedral structure (bphase). Besides, stability in air of the antimonene samples prepared by van der Waals epitaxy was also confirmed using a bunch of techniques including optical microscopy, AFM, Raman, and XPS. Finally, transistor devices were fabricated using few-layer antimonene polygons with thicknesses of 30, 40 and 50 $\mathrm{nm}$ on mica, using gold as contact electrodes and hafnium oxide as a top gate dielectric. The electrical conductivity of the few-layer antimonene polygons was calculated to be $1.610^{4} \mathrm{~S} \mathrm{~m}{ }^{1}$, a typical conductivity value for semimetals. Additionally, the samples also showed wavelength independent high transparency in the visible light range. In summary, the work by Ji et al. envisioned interesting potential applications of few-layer antimonene as flexible transparent conductive electrodes and paved the way for further experimental investigations on the extraordinary properties of antimonene, as well as various applications. $^{33}$ 

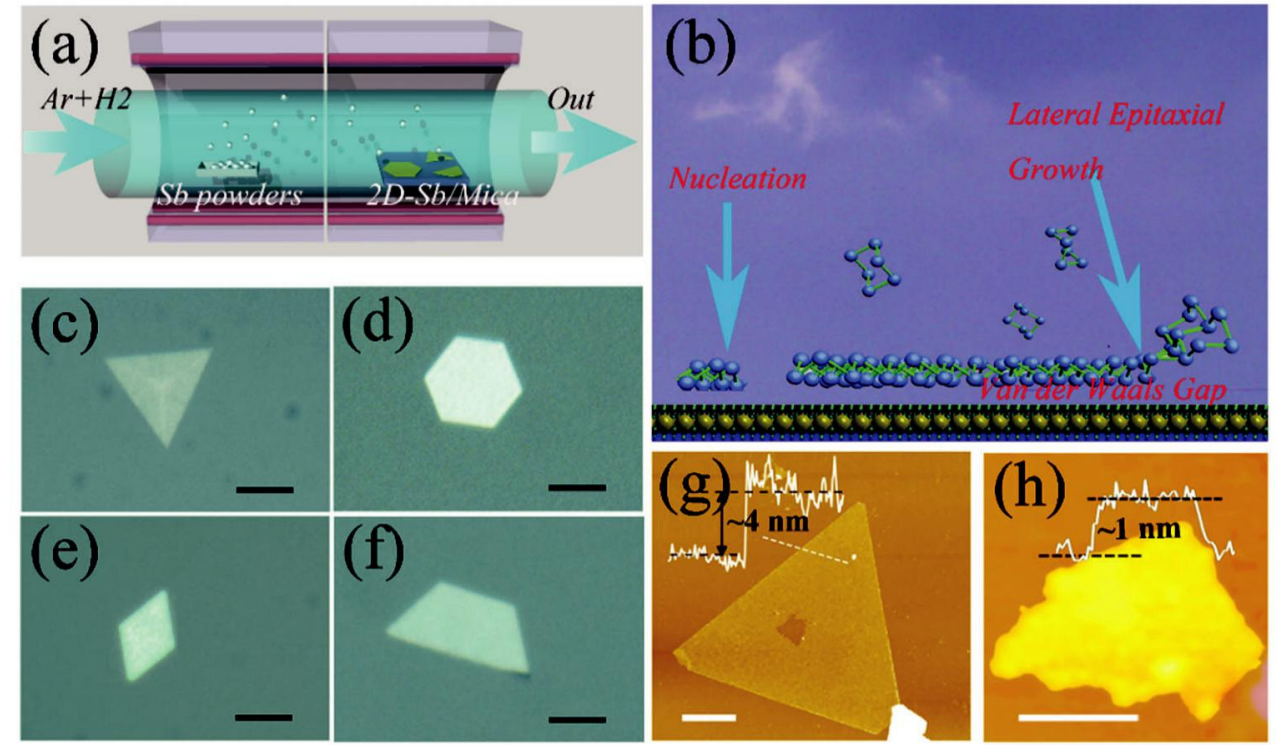

Fig. 15 (a) Schematic illustration of the sample synthesis configurations. (b) Schematic diagram of van der Waals epitaxy. (c-f) Optical images of typical antimonene polygons with triangular, hexagonal, rhombic, and trapezoidal shapes, respectively. The scale bar is $5 \mathrm{~mm}$. (g) AFM image of a typical triangular antimonene sheet. The thickness is $4 \mathrm{~nm}$. The scale bar is $1 \mathrm{~mm}$. (h) AFM image of a tiny antimonene sheet. The thickness is ca. $1 \mathrm{~nm}$. The scale bar is $50 \mathrm{~nm}$. Reproduced from ref. 33 with permission from Nature Publishing Group, copyright 2016.

Molecular beam epitaxy. Micromechanical exfoliation provides a proof of concept for the existence of antimonene as a 2D material. However, large quantity synthesis using this procedure is impossible. Epitaxial synthesis is a well-known scalable method to produce significant amounts of crystalline 2D materials. Epitaxial growth and air-stability of monolayer antimonene on $\mathrm{PdTe}_{2}$ has been reported by Wu et al., as shown in Fig. 16a-f. ${ }^{34}$ A schematic drawing illustrates the molecular beam epitaxy (MBE) fabrication process in Fig. 16a. STM topographic images in Fig. 16d and e show antimonene islands in ultra-high vacuum. When the sample is exposed to atmospheric conditions, the STM images do not show substantial contamination confirming the environmental stability of antimonene. Fig. 16b shows a large antimonene area with a well-defined LEED pattern corresponding to the 11 orientation (inset). The apparent height of this antimonene adlayer is about $2.8 \AA$ (Fig. 16f), as measured by the profile line across the edge of the antimonene film (corresponding to the red line in Fig. 16b). This height is close to that of single layer antimonene in the Sb bulk. Consequently, these measurements indicate that the antimony adlayer is a monolayer. A high-resolution STM image of monolayer antimonene is displayed in Fig. 16c, in which the graphene-like honeycomb lattice can be clearly observed. Specifically, a buckled configuration of this honeycomb lattice can be well distinguished. The distance between hexagon centres is $0.413 \mathrm{~nm}$, which is quite close to the periodicity of the $\mathrm{PdTe}_{2}$ substrate $(4.100 .02 \AA)$. Therefore, the close match of these lattices, shown from STM observations, is consistent with the LEED pattern showing (1 1 1) diffraction points (inset in Fig. 16b).

XPS measurements were also performed, indicating that the interaction between monolayer antimonene and the substrate is via a weak van der Waals force and that no chemical interfacial coupling between antimonene and the $\mathrm{PdTe}_{2}$ substrate exists. In summary, the work by $\mathrm{Wu}$ et al. provides an effective method to produce high-quality monolayer antimonene that is chemically inert. ${ }^{34}$ 

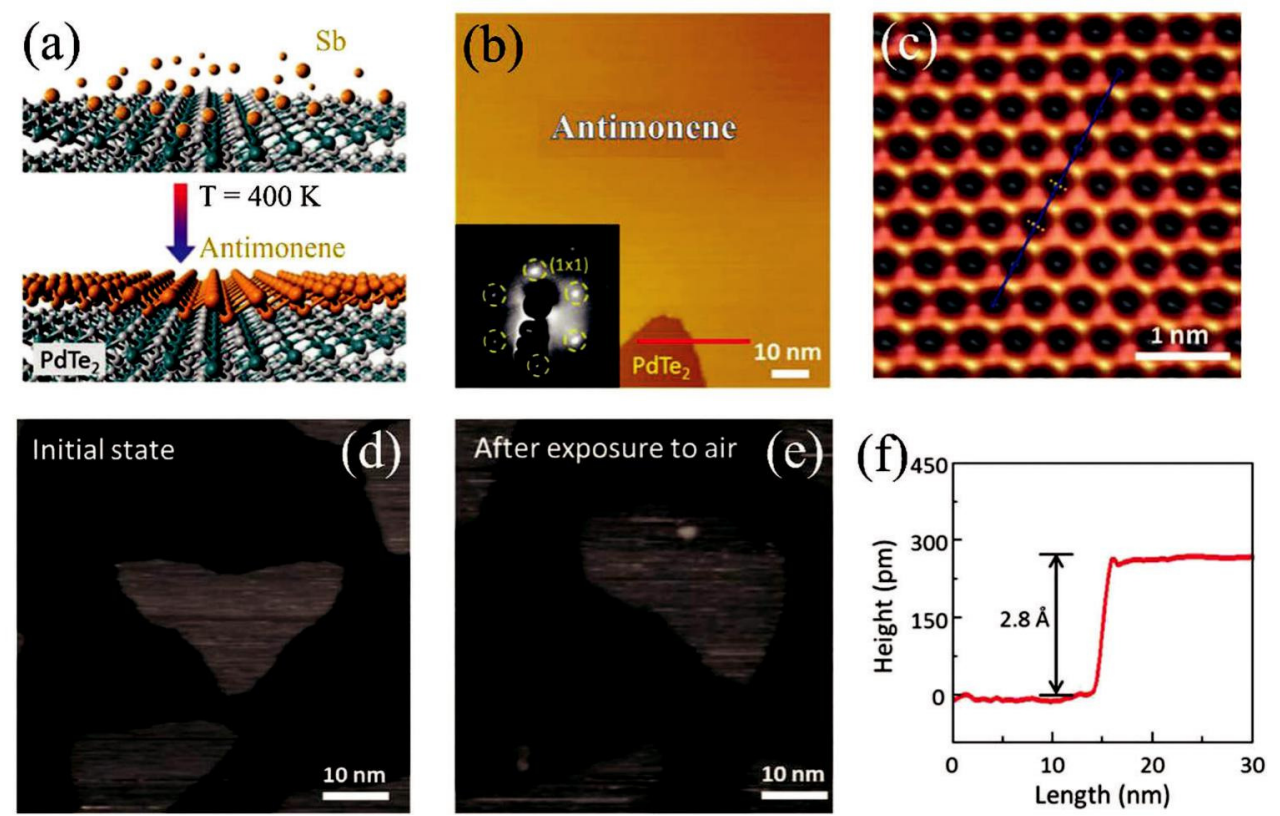

Fig. 16 (a) Schematic of fabrication. (b) STM topographic image ( $2.0 \mathrm{~V}, 10 \mathrm{pA}$ ) of large antimonene island on PdTe 2 . Inset: LEED pattern of antimonene on $\mathrm{PdTe}_{2}$. The six diffraction spots are due to the antimonene $\left(\begin{array}{ll}1 & 1\end{array}\right)$ structure with respect to the substrate. (c) Atomic resolution STM image ( $1.5 \mathrm{~V}, 200 \mathrm{pA})$ of monolayer antimonene with enhanced visibility showing the graphene-like honeycomb. (d) Typical STM image ( $2.0 \mathrm{~V}, 100 \mathrm{pA}$ ) of antimonene islands on the $\mathrm{PdTe}_{2}$ substrate before air exposure. The surface of the islands is smooth without impurities. (e) STM image (2.0 V, $100 \mathrm{pA}$ ) of the same sample after exposure to air for 20 min. (f) A height profile along the red line in (b), showing that the apparent height of the antimonene island is $2.8 \AA$. Reproduced from ref. 34 with permission from Wiley, copyright 2017.

Electrochemical exfoliation of antimonene. Electrochemical exfoliation has been realized in synthesis of graphene, ${ }^{279,280} \mathrm{MoS}_{2},{ }^{281}$ and phosphorene. ${ }^{282}$ Compared with other methods, the electrochemical approach can potentially have a lower cost for obtaining large-scale 2D materials. Moreover, this method avoids the use of several chemicals by taking advantage of electrochemical activation, presenting an easier purification step. ${ }^{283} \mathrm{Lu}$ et al. reported the electrochemical exfoliation of few-layer antimonene. ${ }^{81}$ As shown in Fig. 17a, an antimony crystal is directly connected to the conducting wire of a DC power supply while the counter electrode is made of Pt wire with a diameter of $0.5 \mathrm{~mm}$. And $\mathrm{Na}_{2} \mathrm{SO}_{4}$ aqueous solution is used as the electrolyte. The antimony crystal and Pt wire form the working electrode and are partially placed into the electrolyte in order to avoid electrolysis and contamination of the connected conducting wire. The whole process is carried out at a $6 \mathrm{eV}$ constant voltage for $60 \mathrm{~min}$ to ensure full intercalation and exfoliation of $\mathrm{Na}$ ions inside cathodic $\mathrm{Sb}$. Then, the electrolyte with exfoliated $\mathrm{Sb}$ nanosheets is centrifuged at a speed of $6000 \mathrm{rpm}$ for $30 \mathrm{~min}$. The sediment after centrifugation is washed by ultrasonication in deionization water for $10 \mathrm{~min}$. The two processes, centrifugation and ultrasonicationwashing, have to be repeated three times. Finally, the dispersion solution is dried at $601 \mathrm{C}$ for $24 \mathrm{~h}$ in a vacuum and diluted in NMP for characterization. As illustrated in Fig. 17b, the height and phase of the synthetized $\mathrm{Sb}$ nanosheets are characterized by AFM with samples dispersed on $\mathrm{Si} / \mathrm{SiO}_{2}$ substrates. The AFM image shows flakes with $\sim 31.6 \mathrm{~nm}$ in thickness with a smooth surface and an irregular profile. The microstructure is characterized by TEM using a FEI Tecnai G2F30 field-emission TEM equipment operated at $300 \mathrm{kV}$. The TEM image in Fig. 17c presents a lateral size of $\sim 10.3 \mathrm{~mm}$ and the highresolution TEM image in Fig. 17d further exhibits a rigid arrangement of lattice planes. The observed interdistance of the lattice fringes is $0.228 \mathrm{~nm}$, in conformity to the (100) interplanar distance of 
rhombohedral gray antimony $(0.225 \mathrm{~nm}) .^{33}$ The Raman spectrum is obtained by the Renishaw method via a confocal Raman microscope. In Fig. 17e, the bulk antimony crystal exhibits the typical sharp scattering peaks of the $\mathrm{E}_{\mathrm{g}}$ and $\mathrm{A}_{1}$ vibration modes (red dashed line), ${ }^{33}$ situating at 111.7 and $149.7 \mathrm{~cm}^{-1}$, respectively. And for multilayer antimonene of $31.6 \mathrm{~nm}$, the peak positions of $\mathrm{E}_{\mathrm{g}}$ and $\mathrm{A}_{1}$ (the blue lines) are blueshifted to 113.8 and $150.9 \mathrm{~cm}^{-1}$, respectively. Additionally, the intensity ratio of the $\mathrm{E}_{\mathrm{g}}$ and $\mathrm{A}_{1}$ peaks reduces from 0.07 to 0.068 upon thinning the $\mathrm{Sb}$ crystal to $31.6 \mathrm{~nm}$. X-ray photoelectron spectroscopy (XPS) shows a sharp symmetric photoelectron peak at $528 \mathrm{eV}$, in agreement with the $\mathrm{Sb}-\mathrm{Sb}$ $3 \mathrm{~d}_{5 / 2}$ orbital bonding (Fig. 17e). The presence of only one peak indicates that few-layer antimonene has a nonoxidation feature. In summary, the work by Lu et al. demonstrates a facile, green and cost-effective multiple electrochemical exfoliation method for few-layer antimonene. ${ }^{81}$

(a)

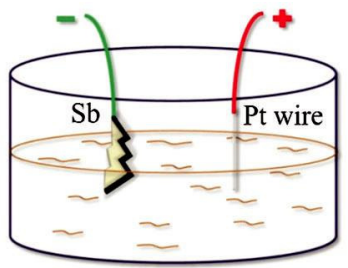

(c)

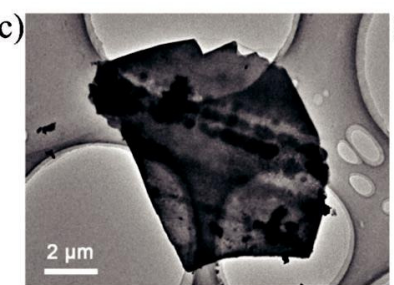

(e)

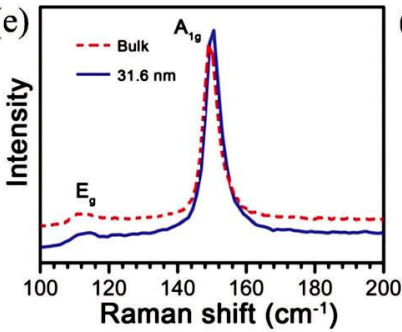

(b)

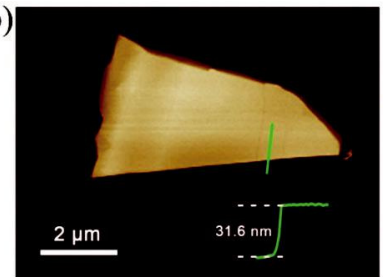

(d)

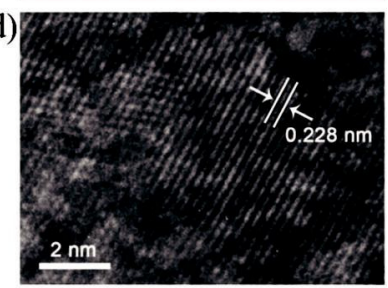

(f)

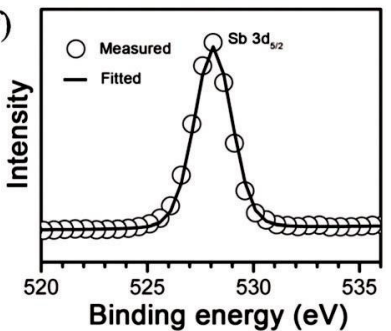

Fig. 17 Characterization of the prepared multilayer antimonene. (a) Schematic illustration of the two-electrode system used for the electrochemical exfoliation of antimony, with an antimony crystal, a Pt wire and $\mathrm{Na}_{2} \mathrm{SO}_{4}$ aqueous solution as the working electrode, counter electrode, and electrolyte, respectively. (b) Typical AFM image of an electrochemically exfoliated multilayer antimonene nanoflake. (c) TEM and (d) HRTEM images of the multilayer antimonene flake. (e) Raman spectra of bulk antimony and $31.6 \mathrm{~nm}$ thick multilayer antimonene shown in (b). (f) XPS spectrum of the $\mathrm{Sb} 3 \mathrm{~d}_{5 / 2}$ peak of exfoliated multilayer antimonene. Reproduced from ref. 81 with permission from Wiley, copyright 2017.

\subsection{Fabrication of bismuthene}

Bulk bismuth, a layered rhombohedral crystal, is a semi-metal with a very small indirect band overlap and unique electronic properties resulting from a tiny electron effective mass of about $0.001 \mathrm{~m}_{0}$ and low charge density ${ }^{284} 2 \mathrm{D}$ bismuth materials have received steadily growing interest due to their quantum size effects, long mean free path, low carrier density and large spin- orbit coupling. ${ }^{285-287}$ In particular, realizing bismuthene as a 2D topological insulator will be of great advantage in monoelement materials. In fact, the synthesis of bismuth thin films has been reported and can be traced back to $1966 .{ }^{288}$ High 
quality bismuth films have been grown on $\mathrm{Si}(111)$ and $\mathrm{Si}(001)$ substrates by using molecular beam eptiaxy (MBE) and incorporation of an annealing step into the growth process. ${ }^{246-249,251}$

Sun et al. grew buckled bismuth nanosheets on a $\mathrm{NbSe}_{2}$ superconducting substrate. ${ }^{251} \mathrm{The} \mathrm{Bi} / \mathrm{NbSe}_{2}$ system has a weak interaction, and the ultrathin bismuth material shows a quasi-freestanding feature. The STM image of $\mathrm{Bi}(111)$ is shown in Fig. 18a. Lu et al. have combined DFT calculations and scanning tunneling microscopy/spectroscopy (STM/STS) experiments to study the topological properties of puckered bismuth nanosheets (Fig. 18b). ${ }^{252}$ It should be mentioned that bulk bismuth only has the layered rhombohedral bulk bismuth with buckled forms, but 2D bismuthene with the puckered structure can been fabricated using suitable substrates. Walker et al. reported the first direct dry transfer of 2D bismuth nanosheets grown through the MBE method (Fig. 18c). ${ }^{253} 2 \mathrm{D}$ bismuth nanosheets are successfully transferred by a double cantilever beam fracture technique. Importantly, the transferred 2D bismuth material can maintain electrical, optical, and structural properties comparable to the as-grown epitaxial 2D bismuth material. The ability to easily transfer epitaxial materials can enable previously impossible measurements of the electronic and spintronic properties of 2D group-VA materials.

Reis et al. have realized monolayer bismuthene, which is epitaxially deposited on a SiC(0001) substrate (Fig. 18d-f). ${ }^{83}$ They first prepared the SiC substrate starting from n-doped Si-terminated $4 \mathrm{H}-$ $\mathrm{SiC}(0001)$ substrates by dry-etching in a hydrogen-based gas atmosphere. Remarkably, a fully planar graphene-like configuration has been generated instead of buckled bismuthene, which is due to the SiC substrate strain effect (Fig. 18d). The flakes are further characterized by STM, as shown in Fig. 18e. The close-up STM images show the detailed honeycomb structure of bismuthene. The monolayer-substrate composites are detected to have a wide gap and conductive edge states by scanning tunneling spectroscopy. Importantly, the $\mathrm{SiC}(0001)$ substrate not only stabilizes the planar bismuthene monolayer but also plays a key role in achieving a large bulk gap of approximately $0.8 \mathrm{eV}$.

Other groups have reported other methods to fabricate free-standing buckled bismuthene. Hussain et al. developed an efficient hot-pressing method to fabricate bismuthene from Bi nanoparticles. ${ }^{84}$ The $\mathrm{Bi}$ nanoparticles are compressed using suitable press while heating at optimized temperature. By using atomic force microscopy, the minimum thickness is figured out to be $2.55 \mathrm{~nm}$, indicating the few-atomiclayer of bismuth. Through field emission scanning electron microscopy, Bi nanosheets can be observed with the lateral dimension of up to several micrometers. Lu et al. employed a sonochemical exfoliation method to synthetize high-quality bismuthene with enhanced stability. ${ }^{85}$ First, bulk Bi is ground into powders with isopropyl alcohol. Then, the solution added with more isopropyl alcohol is transferred into a spiral glass bottle and kept under ice-bath sonication and then sonicated for $10 \mathrm{~h}$. The suspension is centrifuged at $5000 \mathrm{rpm}$ for $20 \mathrm{~min}$. The AFM image of fabricated bismuthene shows that the nanosheets have a height of $\sim 4 \mathrm{~nm}$ with a smooth surface. 


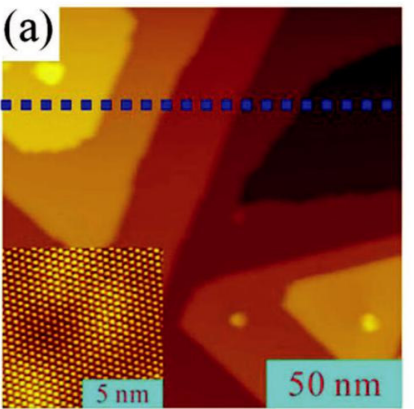

(d)

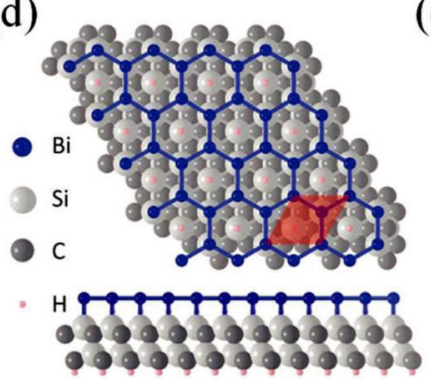

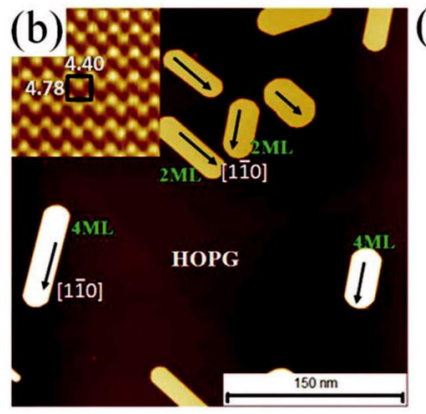

(e)

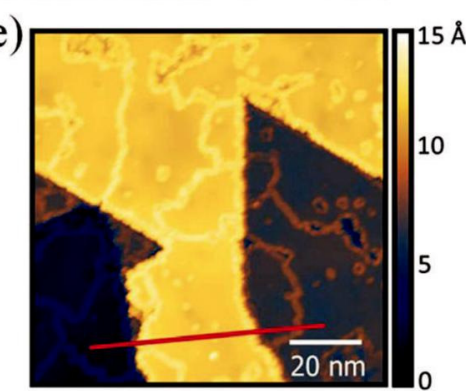

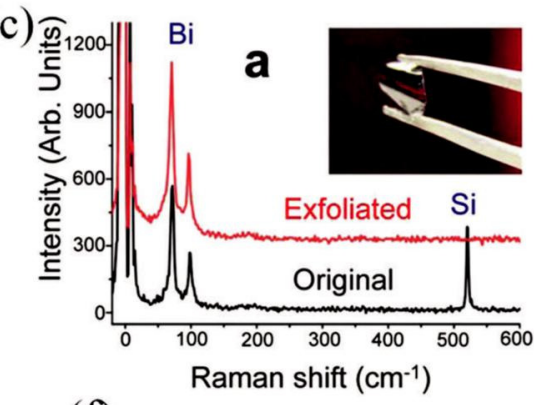

(f)

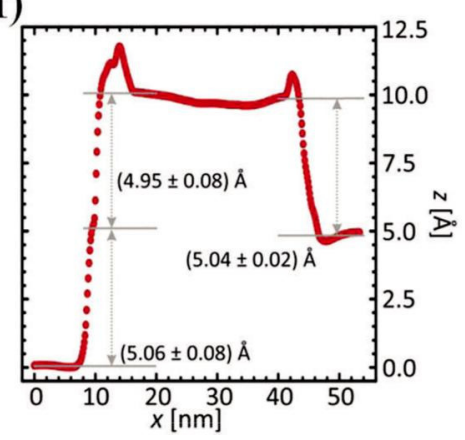

Fig. 18 (a) STM image of $\mathrm{Bi}(111)$ on $\mathrm{NbSe}_{2}$. Inset: Atomic resolution image taken from the surface. Reproduced from ref. 251 with permission from the American Chemical Society, copyright 2017. (b) STM image of the Bi(110) film on HOPG. Most of the Bi films are islands with a preferred elongation direction along [110], as indicated by black arrows. The inset shows an atomicresolution STM image of the film surface. The surface unit cell is indicated by the black rectangle with measured lattice constants in $\AA$ A. Reproduced from ref. 252 with permission from the American Chemical Society, copyright 2015. (c) Raman spectrum of Bi exfoliated onto flexible epoxy through mechanical peeling. Inset: Photograph of flexible Bi on epoxy post-exfoliation.Reproduced from ref. 253 with permission from the American Chemical Society, copyright 2016. (d) Sketch of a bismuthene layer placed on the threefold-symmetric $\mathrm{SiC}(0001)$ substrate in R30 commensurate registry. (e) Topographic STM overview map showing that bismuthene fully covers the substrate. The flakes are of thickness $\sim 25 \mathrm{~nm}$, limited by domain boundaries. (f) Substrate step height profile, taken along the red line in (e). The step heights correspond to SiC steps. (d-f) Reproduced from ref. 83 with permission from Science, copyright 2017.

\section{Applications of 2D group-VA semiconductors}

\subsection{Field effect transistors}

One of the most important and widely used applications of semiconductors is FET, which is the indispensable building block of modern integrated circuits. In the FETs made from 2D materials instead of 3D materials, there are no dangling bonds, undesirable coupling with phonons and the creation of interface states, which reduce the electrical devices' performances. ${ }^{7}$ Semiconducting group-VA crystals show their potential as the body terminal of FETs. As channel materials of FETs, compared with graphene and $\mathrm{MoS}_{2}$, black phosphorene simultaneously has two main advantages, high on/off ratio for effective switching and charge carrier mobility for fast operation. In 2014, Liu et al. ${ }^{112}$ and Li et al. ${ }^{16}$ firstly reported phosphorene field-effect transistors based on few-layer phosphorene (Fig. 19a). The drain current modulation of the transistor is on the order of $10^{5}$ at room temperature, four orders of magnitude larger than that in graphene. The highest value of charge carrier mobility is up to $\sim 1000 \mathrm{~cm}^{2} \mathrm{~V}^{-1} \mathrm{~s}^{-1}$, superior to that of commercial silicon-based devices. Li et al. also pointed out that optimizing the gate dielectric could improve the existing low on-state current and high subthreshold swing in the future. ${ }^{16}$ Chen et al. reported fabrication of a FET device made of sandwiched heterostructures by encapsulating 
few-layer phosphorene between hexagonal boron nitride layers to realize ultra-clean interfaces. ${ }^{197}$ This electronic device allowed a higher mobility of up to $\sim 1350 \mathrm{~cm}^{2} \mathrm{~V}^{-1} \mathrm{~s}^{-1}$ at room temperature and on-off ratios exceeding $10^{5}$.
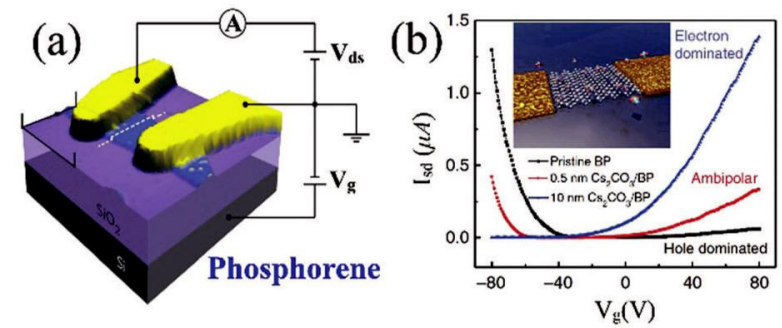

(c)

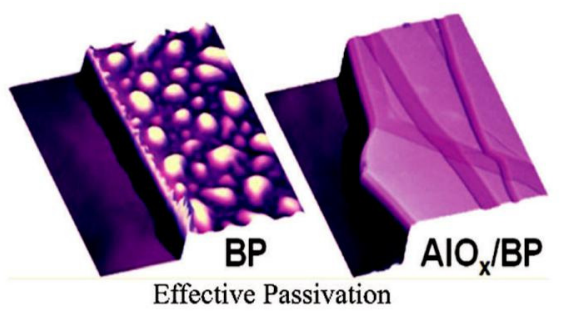

(d)

Arsenene and antimonene

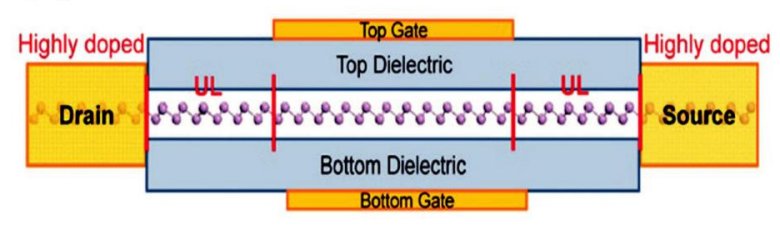

(e)

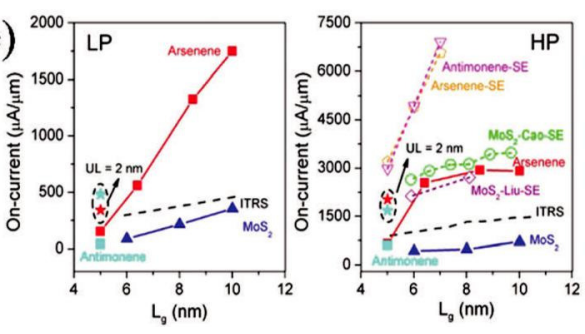

Fig. 19 (a) Schematic of the device structure of a few-layer phosphorene FET. Reproduced from ref. 16 with permission from Nature Publishing Group, copyright 2014. (b) Linear plot of the transfer curves at 0.5 and $10 \mathrm{~nm} \mathrm{Cs}_{2} \mathrm{CO}_{3}$ coverage with respect to pristine black phosphorus. The electron transport of the black phosphorus FET was significantly improved after $\mathrm{Cs}_{2} \mathrm{CO}_{3}$ modification, leading to either a more balanced ambipolar or even electron-transport-dominated FET characteristic. Inset: Schematic illustration of the black phosphorus device coated with $\mathrm{Cs}_{2} \mathrm{CO}_{3}$. Reproduced from ref. 302 with permission from Nature Publishing Group, copyright 2015. (c) Micrographic images of unencapsulated and $\mathrm{AlO}_{\mathrm{x}}$ encapsulated phosphorene. Reproduced from ref. 222 with permission from the American Chemical Society, copyright 2014. (d) Schematic of arsenene and antimonene double-gated MOSFETs. (e) Optimal on-current versus gate length for the arsenene (red and orange), antimonene (cyan and magenta), doublegated n-MOSFETs in the low power (LP) and high performance (HP) applications. Black dashed lines represent the International Technology Roadmap for Semiconductors (ITRS) requirements. (d and e) Reproduced from ref. 66 with permission from the American Chemical Society, copyright 2017.

Based on theoretical and experimental studies, it is shown that the carrier mobilities of black phosphorene are highly direction dependent, because of the structural anisotropy. ${ }^{18,112,289-292}$ The lower effective masses along the armchair direction lead to higher drive current at the same biasing. At the same time, the higher degree of anisotropy improves the performance of p-type devices. ${ }^{289}$ In addition, some groups also indicated that the device transport characteristics depend on the transport directions by the use of the non-equilibrium Green's function formalism. ${ }^{293-295}$ It is demonstrated that this transport anisotropy helps improve the on-state current with the channel length below $10 \mathrm{~nm}$ and meeting the ITRS target in the presence of a quantum tunneling effect. ${ }^{295}$ However, phosphorene transistors suffer from a few disadvantages, including the strong asymmetric ambipolar characteristics, the high Schottky barrier, and severe surface degradation. It is known that ambipolar channel materials with both $\mathrm{n}$ - and p-type transport are preferred in Complementary Metal-Oxide-Semiconductor Transistor (CMOS) logic circuits in order to simplify the circuit design and also to save the layout area. ${ }^{296}$ The ambipolar characteristics of phosphorene transistors are observed, ${ }^{16,224,297-301}$ but the ambipolar behavior is strongly asymmetric which is unfavorable for complementary logic devices. In order to balance the ambipolar behavior, some studies focused on enhancing the slower hole transport, by optimization of the device structure, fabrication conditions and flake thickness. ${ }^{302-304}$ Xiang et al. reported an effective modulation on ambipolar characteristics of phosphorene transistors through in situ surface functionalization with $\mathrm{Cs}_{2} \mathrm{CO}_{3}{ }^{302}$ As 
shown in Fig. 19b, with a coverage of $0.5 \mathrm{~nm} \mathrm{Cs} \mathrm{CO}_{3}$, the on-current in the electron regime almost reaches the same level as that of the hole regime, demonstrating a more symmetric and balanced ambipolar characteristic. The electron mobility is significantly enhanced to be $\sim 27 \mathrm{~cm}^{2} \mathrm{~V}^{-1} \mathrm{~s}^{-1}$ when the thickness of $\mathrm{Cs}_{2} \mathrm{CO}_{3}$ is larger than $10 \mathrm{~nm}$, indicating greatly improved electron transport behavior. The Schottky barrier is generally derived from the difference of work function between metal electrodes and semiconductors, which blocks the electron charge carrier injection. Using an electrode with a suitable work function is an effective method to enhance the device performance. Kamalakar et al. used $\mathrm{TiO}_{2} / \mathrm{Co}$ contacts to get a reduced Schottky barrier below $50 \mathrm{meV}$, which can be tuned further by the gate voltage. ${ }^{305}$ In addition, good transistor performance is achieved in the device, with $\mathrm{I}_{\text {on }} / \mathrm{I}_{\text {off }} 410^{4}$ and a mobility of $155 \mathrm{~cm}^{2} \mathrm{~V}^{-1} \mathrm{~s}^{-1}$ for hole conduction at room temperature.

While exposed to air, phosphorene irreversibly reacts with $\mathrm{O}_{2}$ and $\mathrm{H}_{2} \mathrm{O}$ to form phosphoric acid or oxidized phosphorus compounds over a short period of time. ${ }^{217,222,298}$ The surface degradation brings about physical changes such as volume expansion and uneven surfaces, chemical changes such as the electronic structure and large Schottky barrier heights, thus degrading the mobility. ${ }^{112,190,306}$ Many effective approaches were reported to attempt to reduce the surface degradation, including passivation and solvent exfoliation. By capping the flake with a thick $\mathrm{AlO}_{\mathrm{x}}$ layer ${ }^{22,300,306-308}$ or direct liquid exfoliation in anhydrous and oxygen-free organic solvents, ${ }^{228,230-232,309}$ phosphorene transistors can maintain the ambient stability for a long time, overcoming a critical material challenge for applied research and development. Fig. 19c shows the significant difference between pristine phosphorene and phosphorus nanosheets capped with $\mathrm{AlO}_{\mathrm{x}}$ overlayers upon exposure to ambient conditions, and phosphorus nanosheets deposited with $\mathrm{AlO}_{\mathrm{x}}$ overlayers effectively suppress ambient degradation. In addition, Chen et al. encapsulated few-layer black phosphorene between hexagonal boron nitride layers to ensure the high quality of phosphorene under ambient conditions. ${ }^{197}$

The other two 2D group-VA materials, buckled arsenene and antimonene, have been successfully synthesized. Importantly, different from phosphorene, they are highly stable when exposed to ambient conditions. Additionally, monolayer arsenene and antimonene show a wide band gap based on DFT calculations, indicating their potential for FET applications. Pizzi et al. ${ }^{36}$ and Wang et al. ${ }^{66}$ theoretically predicted the performance of sub-10 nm monolayer arsenene and antimonene metal oxide semiconductor FETs (MOSFETs). Using Takagi's formula with SOC effects, Pizzi et al. calculated that the electron mobility and the hole mobility are 635 and $1700 \mathrm{~cm}^{2} \mathrm{~V}^{1} \mathrm{~s}{ }^{1}$ for As, and 630 and $1737 \mathrm{~cm}^{2} \mathrm{~V}^{1} \mathrm{~s}{ }^{1}$ for $\mathrm{Sb}$, respectively. ${ }^{36}$ In particular, the hole mobility is significantly enhanced by SOC, increasing by $25 \%$ and $84 \%$ in As and Sb, respectively. Instead, in arsenene and antimonene the SOC effects are negligible for the conduction band. Supported by the high mobility, they performed a full-device simulation of FETs based on arsenene and antimonene as channel materials within the non-equilibrium Green function formalism. Significantly, arsenene and antimonene show a performance that is compliant with industry requirements for ultra-scaled devices in the sub-10 nm scale. In 2017, Wang et al. reported that arsenene and antimonene MOSFETs based on the ab initio quantum transport approach show excellent device performances. ${ }^{66}$ As shown in Fig. 19d and e, both the low power and high performance of monolayer arsenene MOSFETs surpass the monolayer $\mathrm{MoS}_{2}$ Schottky barrier FETs on all the sub-10 nm scales in terms of an obvious advantage of large $\mathrm{I}_{\mathrm{on}}$. They can satisfy both the low power and high performance application requirements for all the studied nodes, down to $5 \mathrm{~nm}$, for the next decade in ITRS 2013. All these works indicate that arsenene and antimonene are very attractive for nanoscale electronic devices.

\subsection{Photodetectors}

Black phosphorene has attracted tremendous attention for optoelectronic and photonic applications because of its unique in-plane anisotropy, thickness-dependent direct band-gap and high carrier 
mobility. ${ }^{232,310}$ The carrier mobility of phosphorene-based FETs is as high as $286-1000 \mathrm{~cm}^{2} \mathrm{~V}^{-1} \mathrm{~s}^{-1}$, together with tunable band gaps and anisotropic carrier transport properties, ${ }^{16,18}$ indicating that phosphorene can be employed as a suitable candidate for infrared optoelectronic applications that fills the gap between graphene and large-band-gap TMDCs. ${ }^{297}$ Furthermore, Li et al. achieved hole mobility as high as $3900 \mathrm{~cm}^{2} \mathrm{~V}^{-1} \mathrm{~s}^{-1}$ in phosphorene-FET with h-BN as the top and bottom gate dielectric materials. ${ }^{311}$ In order to satisfy the infrared applications, the carrier mobility and band gap can be controlled by nano-engineering, defect engineering, strain and chemical treatments. ${ }^{146,304,312,313}$ In addition, the infrared optical properties and the related band transitions of multilayer phosphorene was revealed by Fourier transform infrared spectroscopy (FTIR), which indicates the stupendous potential of phosphorene in infrared photodetection. ${ }^{314}$

According to the band gap of black phosphorene, it can be configured as an excellent UV photodetector with high detectivity and photoresponsivity due to the resonant-interband transition between two specially nested valence and conduction bands (Fig. 20a) ${ }^{315}$ Buscema et al. achieved a broadband phosphorene photodetector in the near infrared regime with a photoresponsivity of $4.8 \mathrm{~mA} \mathrm{~W}{ }^{1}$ based on a phosphorene FET, where the photo-generated carriers emerge and separate in the electrode/phosphorene interface. ${ }^{316,317}$ At the same time, it was reported that few-layer phosphorene can be used as high performance visible photodetectors for high-contrast, diffraction-limited optical imaging. ${ }^{318}$ Compared with other TMDCs, one unique property of phosphorene is the anisotropic carrier transport and light absorption behavior originated from the in-plane asymmetric structure, thus phosphorene can be employed as a broadband, polarization-sensitive photodetector from $400 \mathrm{~nm}$ to 3750 $\mathrm{nm}$ and provide novel functionalities in phosphorene-based optoelectronic devices. ${ }^{319}$ However, this operation mode hasn't optimized the light absorption and photo-carrier separation efficiency. To solve this problem, PN junctions are created via different strategies to effectively separate the photogenerated carriers. Buscema et al. reported the first black phosphorene lateral PN junction by the dual-gating method that drastically enhanced the photoresponsivity. ${ }^{320}$ Furthermore, under the photovoltaic mode, the dark current of the phosphorene PN junction photodetectors is decreased, because no bias voltage is applied (Fig. 20b-e). Moreover, the lateral phosphorene PN junction was also constructed by the chemical doping method and achieved high responsivity and fast response time for near-infrared photodetection ${ }^{321}$ due to the simultaneously modified carrier mobility and transport behaviors. In contrast, vertical PN junctions are also effective due to the larger photo-active area. Deng et al. demonstrated a gate-tunable PN diode based on phosphorene/ $\mathrm{WS}_{2}$ van der Waals heterostructure and achieved a photoresponsivity of $\sim 418 \mathrm{~mA} \mathrm{~W}{ }^{-1} \cdot{ }^{192}$ However, these approaches limit the active area selectivity and increase the fabrication procedures. In addition, the relatively low light absorption coefficient is another obstacle for photodetective applications. As a result, the enhancement of light absorption of phosphorene by optical field controlling is an essential method for phosphorene photodetectors. For example, Youngblood et al. demonstrated obvious absorption enhancement of phosphorene by a silicon waveguide in the phosphorene active area and achieved a photoresponsivity of $657 \mathrm{~mA} \mathrm{~W}^{-1}$ and a detectivity of almost three orders of magnitude since the photodetectors are designed in the photovoltaic operation mode (Fig. 20f). ${ }^{303}$ Similarly, Viti et al. demonstrated efficient room temperature $\mathrm{THz}$ photodetectors combining top-gated FETs exploiting multilayer phosphorene with specially designed $\mathrm{THz}$ asymmetric antennas to increase the light absorption. ${ }^{322}$ Last but not least, the environmental stability of phosphorene is an important factor for phosphorene photodetectors. The device architecture and photonic design strategies, dielectric layer capping ${ }^{222,323}$ and chemical treatment ${ }^{324}$ would be promising ways to integrate with conventional phosphorene photodetectors. It has been demonstrated that the band gap of phosphorene can be widely tuned by the chemical doping method, ${ }^{325}$ owing to the giant Stark effect, which may further extend the working wavelength range to the far-infrared regime. 
As brothers of phosphorene in the group-VA family, arsenene, antimonene and bismuthene cover a wide range of energy band gaps, which are crucial for a broadband photo-response. Combining such a broad range of band gaps and high carrier mobilities, 2D group-VA materials are promising candidates for photodetectors.

(a)

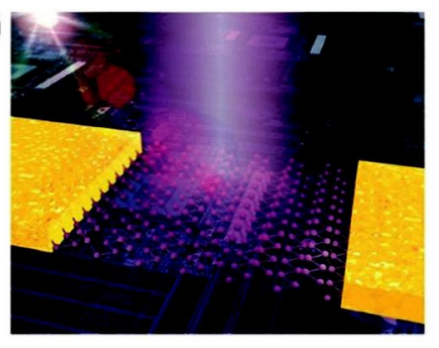

(b)

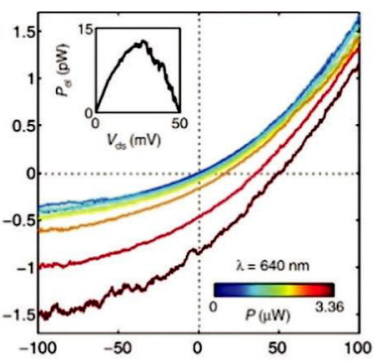

(c)

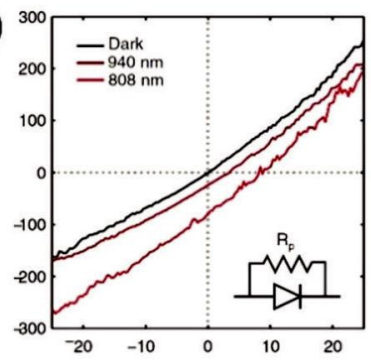

(d)

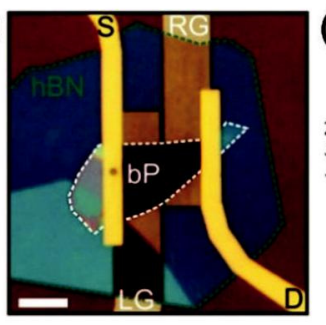

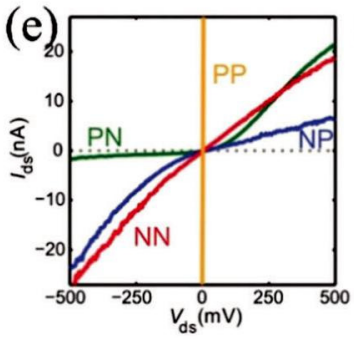

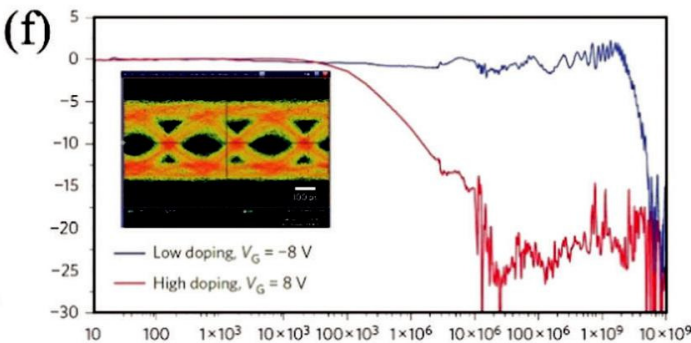

Fig. 20 (a) Three-dimensional view of a few-layer black phosphorus photo-FET. Reproduced from ref. 315 with permission from the American Chemical Society, copyright 2015. (b) Output characteristics in the PN configuration as a function of the incident optical power $(1=640 \mathrm{~nm})$, and the inset shows the electrical power that can be harvested at the maximum employed illumination power. (c) Output characteristics in the PN configuration in darkness (black solid line) and under illumination of different excitation wavelengths at fixed power $(\mathrm{P}=0.33 \mathrm{~mW}$ ), and the inset shows the schematics of the equivalent circuit. (d) Optical image of one of the fabricated devices. (e) $I_{\mathrm{ds}}-V_{\mathrm{ds}}$ characteristics of the device in different gate configurations. (b-e) reproduced from ref. 320 with permission from Nature Publishing Group, copyright 2014. (f) The response of the phosphorene photodetector is measured when phosphorene is gated to low and high doping. At low doping, the response is broadband with a cutoff frequency of $3 \mathrm{GHz}$, which is limited by the RC bandwidth of the contact pads and the input impedance of the preamplifier. At high doping, the response rolls off at $0.2 \mathrm{MHz}$, indicating that the photoresponse is of a thermal origin, as expected from the bolometric effect. The inset shows the receiver eye diagram at a data rate of 3 Gbit s ${ }^{1}$ measured with the phosphorene photodetector. Scale bar, 100 ps. Reproduced from ref. 303 with permission from Nature Publishing Group, copyright 2015. 


\subsection{Light emitting devices}

2D group-IVA materials, such as graphene, silicene, germanene, and stanene, are semimetallic, and the lack of a suitable band gap totally restricts their applications in light-emitting devices. 2D group-VA materials are semiconductors with significant fundamental band gaps, thus rendering them possible candidates for active materials in light-emitting devices.

Phosphorene is an atomically-thin optical material with direct exciton emission, and its wavelength is tunable by controlling the number of layers. Zhang et al. investigated the photoluminescence (PL) spectra of few-layer phosphorene exfoliated on a silicon substrate, obtaining a strong and highly layer-dependent PL in few-layer phosphorene (Fig. 21a). ${ }^{220}$ Strong PL peaks of phosphorene at 961, 1268, 1413, and 1558 $\mathrm{nm}$ have been observed in 2-5 layers, which correspond to energy peaks of $1.29,0.98,0.88$, and $0.80 \mathrm{eV}$, respectively. The PL peaks are attributed to the nature of excitons, which represent lower bounds on the fundamental band gap values in few-layer phosphorene. The measured PL spectra provide very useful information to investigate the exciton nature and the electronic structures in phosphorene. Li et al. studied the PL spectra of monolayer, bilayer, and trilayer phosphorene at $77 \mathrm{~K}$ under unpolarized photoexcitation at $2.33 \mathrm{eV}$ (Fig. $21 \mathrm{~b}$ and c) ${ }^{326}$ They have observed strong polarization dependence in monolayer, bilayer and trilayer phosphorene. The PL peak energy can match well with that in the absorption spectra, which confirms the direct bandgap nature of phosphorene. Additionally, Ge et al. pointed out that the intense and stable PL peaks of the black phosphorene quantum dots have been found in the blue-violet wavelength region. ${ }^{327}$ Due to the excellent PL intensity, stability and its excitation wavelengthindependence in the blue-violet light region, phosphorene is expected to be a suitable material for blueviolet light irradiation sources. Aghaeimeibodi et al. reported a new class of near-infrared emission from defect states in phosphorene. ${ }^{328}$ With the linear increase of excitation intensity, a sublinear increase of PL intensity at $1240 \mathrm{~nm}$ has been observed, which confirms the defect-based nature of this emission. They found that the defect emission is 34 times brighter than the exciton at low temperature, and it still maintains the brightness even up to room temperature. The brighter room-temperature emission is an important advantage for a source of infrared light with potential room-temperature and near-infrared optoelectronic applications.

Interestingly, multilayer arsenene nanoribbons have been fabricated and exhibited green PL light emission at $540 \mathrm{~nm}$ at room temperature (Fig. 21d). ${ }^{29}$ The green light emission implies that the band gap of the multilayer arsenene nanoribbons is about $2.3 \mathrm{eV}$. Two factors lead to their bandgap opening. One is the quantum confinement effect induced by dimensionality reduction, and the other is the turbostratic stacking. In addition, Tsai et al. also fabricated multilayer antimonene nanoribbons with orange light emission at $610 \mathrm{~nm}$ (Fig. 21e). ${ }^{30}$ Thus, the PL measurements of arsenene and antimonene nanoribbons indicate that they also possess potential applications in light-emitting devices.

Large area and highly crystalline bismuth nanosheets have been successfully fabricated by using a novel and facile hot-pressing method. ${ }^{84}$ It has been argued that the significant PL response from ultrathin Bi nanosheets (Fig. 21f) might be attributed to the carrier confinement effects and process-dependent crystal defect/dislocation induced trapping of electrons and excitons in ultrathin BiNSs. 

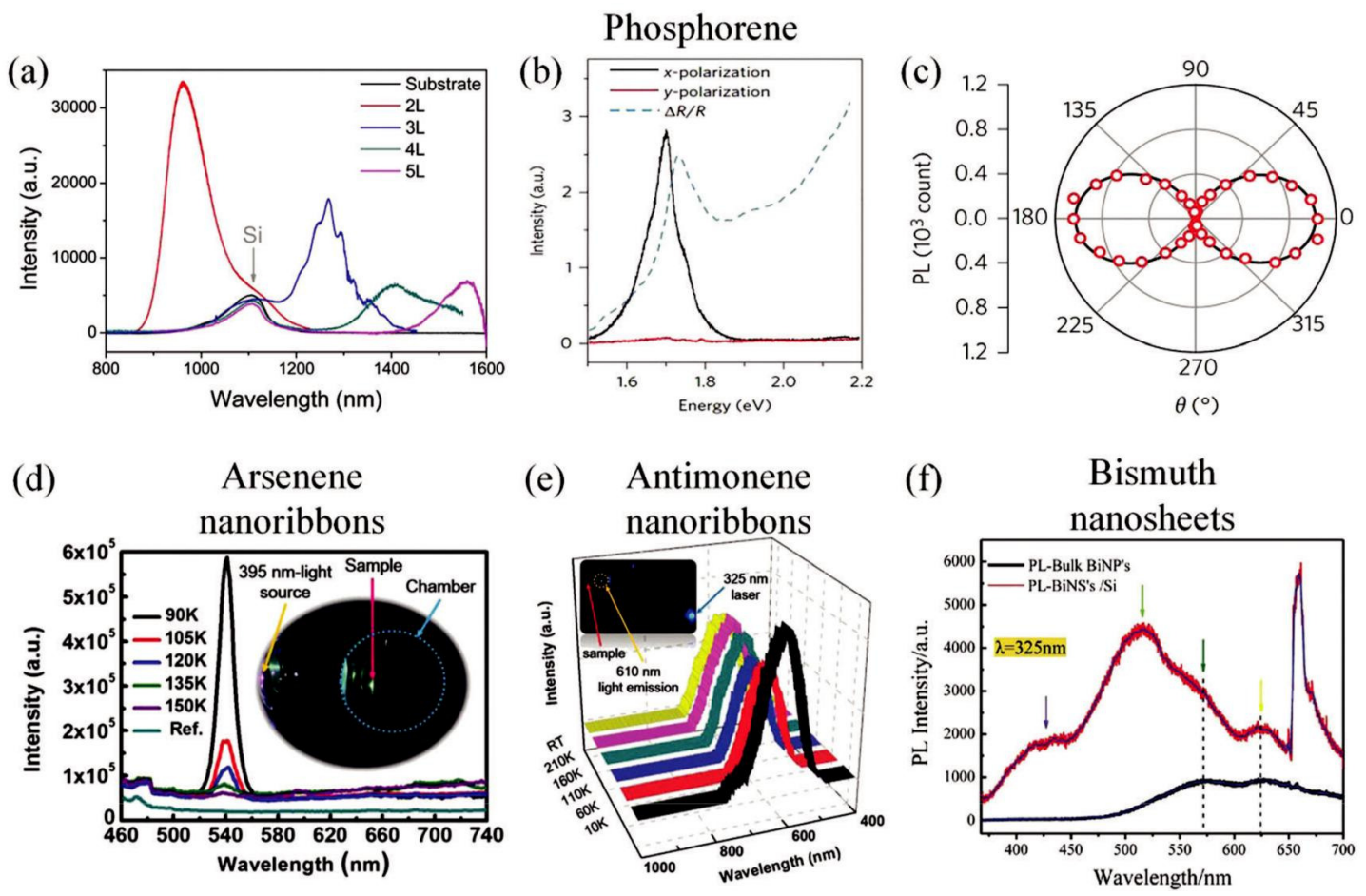

Fig. 21 (a) Photoluminescence spectra of 2L, 3L, 4L and 5L phosphorene. Note: The tiny oscillation on the PL curve of $3 \mathrm{~L}$ is due to the limitation error of the InGaAs detector. Reproduced from ref. 220 with permission from the American Chemical Society, copyright 2014. (b) Photoluminescence spectra of monolayer phosphorene recorded at $77 \mathrm{~K}$ with unpolarized photoexcitation at $2.33 \mathrm{eV}$. (c) Intensity of the PL at peak energy as a function of polarization angle $\mathrm{y}$. Data taken on monolayer phosphorene are shown by the red circles. All angular dependences show a nearly perfect cos $2 \mathrm{y}$ pattern (black solid curves), consistent with the fact that optical transitions along the y direction are forbidden by symmetry. (b and c) Reproduced from ref. 326 with permission from Nature Publishing Group, copyright 2017. (d) PL spectra of multilayer arsenene/InN/InAs at different temperatures. Inset: Photograph of the sample in the chamber of the spectrometer. Reproduced from ref. 29 with permission from the American Chemical Society, copyright 2016. (e) PL spectra of multilayer antimonene/InN/InSb at different temperatures. Inset: Image of the sample in the spectrometer chamber at room temperature. Reproduced from ref. 30 with permission from the Royal Society of Chemistry, copyright 2016. (f) PL spectra of free-standing pristine BiNPs and as-prepared BiNSs on Si substrates, recorded at a wavelength of $325 \mathrm{~nm}$. Reproduced from ref. 84 with permission from Wiley, copyright 2017.

\subsection{Topological spintronic devices}

As a new quantum state of matter, a topological insulator (TI) has attracted extensive research interest due to its bulk insulating gap and topologically protected boundary state. 2D TIs are considered as more promising materials than 3D TIs for spin transport applications because the edge states in the former are more robust against back-scattering than the surface states in the latter. Graphene is the first predicted 2D TI with a quantum spin Hall (QSH) effect. However, due to its weak SOC, graphene has the deficiency of small bulk gap and low-temperature operation. Group-VA elemental monolayers, phosphorene, arsenene, antimonene and bismuthene, are regarded as new emerging 2D materials with unique properties, which have much stronger SOC effect than graphene. As new members in the $2 \mathrm{D}$ material family, can phosphorene, arsenene, antimonene and bismuthene have a TI phase under suitable conditions? The answer is yes.

Liu et al. reported that a normal-to-topological phase transition can be obtained with an applied electric field along the stacking direction of few-layer black phosphorene. ${ }^{17}$ When the electric field increases to $0.3 \mathrm{~V} \AA^{1}$, 4-layer phosphorene will transform from a normal insulator into a topological insulator. The system becomes metallic when the electric field reaches $0.6 \mathrm{~V} \AA^{1}$. This band inversion is 
induced entirely by the field-induced Stark effect rather than by SOC. The tuning of topological behavior under an electric field would lead to the quantum spin Hall effect. In addition, by in-plane time-periodic laser fields, a phosphorene monolayer may undergo Lifshitz transitions to topological insulating phases. ${ }^{329}$ Furthermore, strain fields can also drive band inversion of 2D group-VA crystals around the gamma point. Based on the prediction of first-principles calculations, arsenene and antimonene monolayers can be tuned to be topological insulators under biaxial tensile strain larger than $11.7 \%$ and $14.5 \%$, respectively. ${ }^{40,47}$ Moreover, the buckled configuration of arsenene and antimonene enables them to endure large tensile strains of up to $18.4 \%$ and $18 \%$. Thus, they can achieve the maximum bulk gaps at the max strains, making them 2D topological insulators as promising candidate materials for achieving the QSH effect.

Interestingly, Zhou et al. proposed an alternate practical method to combine both the large intrinsic QSH and anomalous Hall conductivity in a flat honeycomb $\mathrm{Sb}$ or Bi monolayer grown on a ferromagnetic $\mathrm{MnO}_{2}\left(\mathrm{H}-\mathrm{MnO}_{2}\right)$ layer by means of first-principles density functional theory with a simplified tight binding analysis. ${ }^{41}$ The $\mathrm{h}-\mathrm{Sb}$ and $\mathrm{h}-\mathrm{Bi}$ sheets become magnetized because of proximity effects and the Dirac points split into different spin channels. Particularly, in the presence of both intrinsic and Rashba SOC, there is an intrinsic QSH state with a large band gap of $228 \mathrm{meV}$ for h-Sb and $941 \mathrm{meV}$ for h-Bi, and a nearly quantized anomalous Hall state with a large band gap of $\sim 10 \mathrm{meV}$ for both h-Sb and h-Bi. Significantly, it is an efficient way to realize both quantized intrinsic spin Hall state and anomalous Hall conductance state in a single material.

Chemical functionalization is a powerful method to create topological insulators and can be used to explore novel electronic properties. ${ }^{185-189,330}$ Arsenene, antimonene and bismuthene have been modified to be TIs by different chemical functionalization. In 2014, Song et al. predicted a group of 2D topological insulators $\mathrm{BiX} / \mathrm{SbX}(\mathrm{X}=\mathrm{H}, \mathrm{F}, \mathrm{Cl}$ and $\mathrm{Br})$ monolayers, where bulk gaps range from $0.32 \mathrm{eV}$ to a recordbreaking value of $1.08 \mathrm{eV}{ }^{185}$ They found that these large bulk band gaps completely result from the strong spin-orbit interaction of $\mathrm{Bi} / \mathrm{Sb}$ atoms which is related to the $\mathrm{p}_{\mathrm{x}}$ and $\mathrm{p}_{\mathrm{y}}$ orbitals around the two valleys $\mathrm{K}$ and $\mathrm{K}^{0}$ of the honeycomb lattice. They proved that $\mathrm{BiX}$ monolayers with honeycomb structures remain stable even at high temperature. Considering their intriguing features, 2D topological insulators, $\mathrm{BiX} / \mathrm{SbX}$ monolayers, are suitable candidates to realize new quantum devices operating at room temperature.

Ma et al. predicted bismuth, antimony and lead bilayers with methyl-functionalization as 2D topological insulators. ${ }^{186}$ These materials possess suitable quantum spin Hall properties due to protected Dirac type topological helical edge states. Notably, these 2D topological insulators have a large nontrivial bulk gap of about $0.9 \mathrm{eV}$, which are large enough for room temperature application. Lately, Zhang et al. reported that antimonene oxide is a 2D topological insulator with a sizable band gap of $117 \mathrm{meV}{ }^{63}$ Zhou et al. ${ }^{183}$ investigated the topological properties of $\mathrm{Sb}(111)$ monolayers decorated with $\mathrm{H}$ and doped with certain magnetic atoms, which have the properties of quantum spin-quantum anomalous Hall insulators with a band gap of up to $53 \mathrm{meV}$. Soon afterwards, Zhou et al. ${ }^{182}$ further systematically explored the electronic and topological properties of a hydrogenated $\mathrm{Sb}$ monolayer epitaxially grown on a $\mathrm{LaFeO}_{3}$ substrate $\left(\mathrm{Sb}_{2} \mathrm{H} / \mathrm{LaFeO}_{3}\right)$ by first-principles calculations and tight-binding models. In the heterostructure, the exotic quantum spin-quantum anomalous Hall (QSQAH) state is observed and the band gap is opened up to $35 \mathrm{meV}$ exactly around the $\mathrm{E}_{\mathrm{F}}$, which can be enlarged by strain or an electric field. A device based on the QSQAH heterostructure, displayed in Fig. 22a, is designed in which the degrees of freedom of the carriers can be manipulated flexibly, providing a promising route towards applications in electronics, spintronics and valleytronics.

Experimentally, Reis et al. reported bismuthene, a candidate for a high-temperature quantum spin Hall material, with a $0.8 \mathrm{eV}$ bandgap. ${ }^{83}$ Combining theory and experiment, in bismuthene grown on a $\mathrm{SiC}(0001)$ substrate, the substrate not only stabilizes the quasi-2D topological insulator but also plays a 
key role in achieving the large gap, with the strong on-site SOC coming directly into play, which would further improve the on-current and the intrinsic switching speed.

A growing research interest has gradually focused on group-VA 2D nanosheets as superior 2D topological insulators. Various strategies are being proposed to achieve group-V 2D TIs with large bulk gaps, which could be characterized and utilized at room temperature. The present progress in group-VA TIs provides a promising innovative platform to design 2D quantum spin Hall devices.

\subsection{Gas sensors}

The past decade has witnessed the blooming of 2D materials as promising candidates for gas sensors due to their large specific surface area, high surface activities and high electrical conductivities originating from the quantum size effects. The absence of a bandgap dramatically hampers the application of some typical 2D materials, such as graphene, silicene, germanene and others, in semiconductor devices, including gas sensors. In contrast, 2D group-VA materials stand out with moderate band gaps ranging from 0.36 to $2.62 \mathrm{eV}$, accompanied by the emerging new phenomena and fascinating physical properties, which make 2D group-VA materials very attractive for future gas detection systems. ${ }^{213,331-333}$ Kou et al. firstly studied the adsorption of $\mathrm{CO}, \mathrm{CO}_{2}, \mathrm{NH}_{3}, \mathrm{NO}$ and $\mathrm{NO}_{2}$ gas molecules on a phosphorene monolayer based on first-principles calculations coupled with a non-equilibrium Green's function (NEGF) approach. ${ }^{159}$ They found that phosphorene exhibits stronger adsorption of gas molecules with high sensitivity and selectivity than graphene and $\mathrm{MoS}_{2}$, tending to be a more efficient sensor. Furthermore, phosphorene is found to be more sensitive to gas molecules containing nitrogen atoms, such as $\mathrm{NO}$ and $\mathrm{NO}_{2}$. Moreover, the transport feature exhibits distinct responses with a dramatic change of the $\mathrm{I}-\mathrm{V}$ relation before and after gas molecule adsorption on phosphorene; the results displayed in Fig. 22b indicate that the adsorption of gas molecules on phosphorene can cause the change of the current, thus changing the resistance, which can be measured experimentally.

In view of the theoretical studies, Abbas et al. firstly reported on the sensing of $\mathrm{NO}_{2}$ gas on FETs fabricated based on few-layer phosphorene. ${ }^{331}$ They found that no apparent change in the peak before and after targeted gas adsorption can be obtained by using the Raman spectroscopy technology, suggesting the good stability of the $\mathrm{NO}_{2}$ adsorbed multilayer phosphorene system. Phosphorene sensors exhibit excellent sensitivity for detection of $\mathrm{NO}_{2}$ concentration down to $5 \mathrm{ppb}$. Moreover, the conductance of devices shows good recovery after flushing the devices with Ar, indicating reversible adsorption and desorption of $\mathrm{NO}_{2}$. Last year, Cho et al. accurately compared the sensing performances of phosphorene, graphene and $\mathrm{MoS}_{2} \cdot{ }^{332}$ The electrical sensing measurement results show that the sensitivity of phosphorene is about 20 times higher than those of graphene and $\mathrm{MoS}_{2}$, which corroborates with the theoretical DFT simulations by Kou et al. ${ }^{159}$ Response/recovery time, selectivity, molar response factor and adsorption behavior all support the fact that phosphorene is a superior gas sensing material. In addition to $\mathrm{NO}_{2}$, phosphorene is also sensitive to methanol. ${ }^{333}$ The device based on phosphorene is very selective in the presence of other vapors for methanol detection and has long-term stability.

The superior sensing performances found in phosphorene motivate the researchers to explore other 2D group-VA materials. Only theoretical studies about buckled arsenene and antimonene used as gas sensing materials have been reported. ${ }^{39,62}$ Similar to phosphorene, arsenene shows high sensitivity to nitrogenbased gas molecules. ${ }^{173,334} \mathrm{Liu}$ et al. studied the adsorption of $\mathrm{CO}, \mathrm{CO}_{2}, \mathrm{~N}_{2}, \mathrm{NH}_{3}, \mathrm{NO}$ and $\mathrm{NO}_{2}$ molecules on the buckled arsenene monolayer. ${ }^{173}$ Among those gases, the $\mathrm{NO}_{\mathrm{x}}$ adsorbents have the largest charge transfer, indicating a potential greater change in conductivity. Only the adsorption of $\mathrm{NO}_{\mathrm{x}}$ can lead to a magnetic moment of $1 \mathrm{~m}_{\mathrm{B}}$. It is suggested that arsenene is a potential candidate for gas sensing of $\mathrm{NO}$ and $\mathrm{NO}_{2}$ molecules. For the antimonene monolayer, first principles calculations demonstrate that $\mathrm{N}_{2}, \mathrm{CO}_{2}, \mathrm{O}_{2}$, $\mathrm{H}_{2} \mathrm{O}$ and $\mathrm{CO}$ are weakly adsorbed, while other toxic gases, $\mathrm{NH}_{3}, \mathrm{SO}_{2}$ and $\mathrm{NO}$, are strongly adsorbed. ${ }^{69}$ 
The sensitivity of adsorbed molecules towards the electronic properties indicates that monolayer antimonene could be a qualified sensing material for gaseous pollutant detection.

(a)

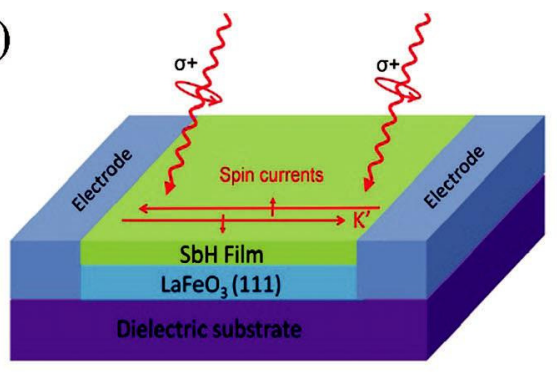

(c)

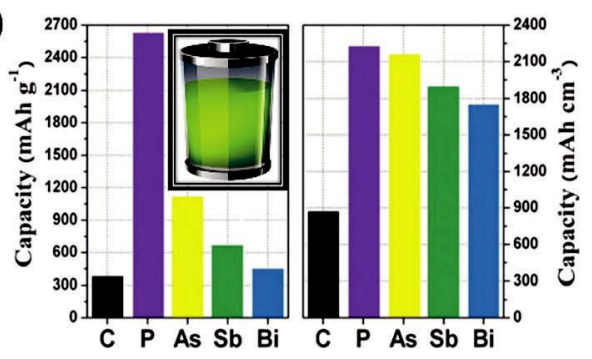

(b)

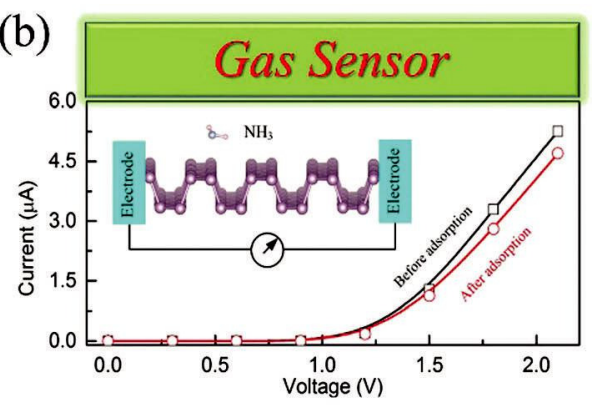

(d)

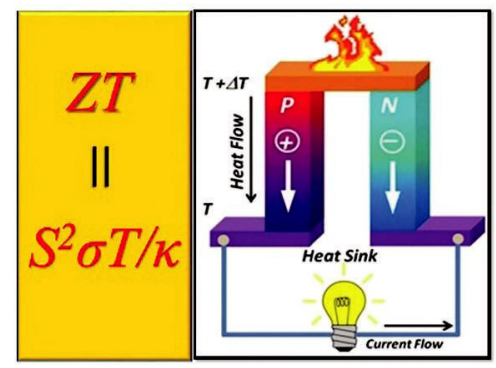

Fig. 22 (a) With the right handed light controls, the spin edge state (red solid arrows) emerges along the edges of the heterostructure. The small solid arrows on the edge states indicate the spin directions. Reproduced from ref. 182 with permission from the American Physical Society, copyright 2016. (b) Phosphorene as a superior gas sensor: selective adsorption and distinct I-V response. Reproduced from ref. 159 with permission from the American Chemical Society, copyright 2014. (c) Gravimetric and volumetric capacities of $\mathrm{C}\left(\mathrm{LiC}_{6}\right), \mathrm{P}\left(\mathrm{Li}_{3} \mathrm{P}\right), \mathrm{As}\left(\mathrm{Li}_{3} \mathrm{As}\right), \mathrm{Sb}\left(\mathrm{Li}_{3} \mathrm{Sb}\right)$ and $\mathrm{Bi}\left(\mathrm{Li}_{3} \mathrm{Bi}\right)$. Reproduced from ref. 335 with permission from Royal Society of Chemistry, copyright 2010. (d) Seebeck effect for power generation; an applied temperature difference causes charge carriers in the material (electrons or holes) to diffuse from the hot side to the cold side, resulting in current flow through the circuit. Reproduced from ref. 349 with permission from Science Direct, copyright 2015. 


\subsection{Battery}

As shown in Fig. 22c, group-VA crystals possess higher theoretical capacity than graphite, indicating that they are promising electrode candidates for $\mathrm{Li}$ and $\mathrm{Na}$ ion batteries. ${ }^{335}$ Compared with $3 \mathrm{D}$ crystals, 2D group-VA materials have extremely high surface areas and superior structural characteristics, exhibiting higher specific capacity. So, what about the performances of 2D group-VA materials as anodes for $\mathrm{Li}$ and $\mathrm{Na}$ ion batteries?

Theoretical calculations show that black phosphorene exhibits ultrafast Li ion diffusion and large capacity for Li ion batteries. ${ }^{336-339}$ Zhang et al. found that Li atoms could form strong binding with $\mathrm{P}$ atoms and stay in the cationic state. ${ }^{336}$ At high concentrations of $\mathrm{Li}$ atoms, the Li-phosphorene complex becomes metallic and gives rise to good electrical conductivity, which is essential for an electrode. Due to the puckered honeycomb nanosheet, the diffusion of $\mathrm{Li}$ atoms on phosphorene is extremely anisotropic. The Li-diffusion along the zig-zag direction is highly energetically favorable, while the diffusion along the armchair direction is almost prohibited. The diffusion barrier of Li atoms along the zig-zag energy is calculated to be $0.08 \mathrm{eV}$, which is much lower than those in two other Li-battery anode materials, graphene $(\sim 0.3 \mathrm{eV})$ and $\mathrm{MoS}_{2}(\sim 0.28 \mathrm{eV}) .{ }^{340-343}$ The low energy barrier leads to diffusivity $10^{4}\left(10^{2}\right)$ times faster than that in graphene $\left(\mathrm{MoS}_{2}\right)$ at room temperature. For comparison, the large energy barrier $(0.68 \mathrm{eV})$ along the armchair direction results in effectively blocked diffusion. Furthermore, the average voltage of the adsorbed Li-atoms is estimated to be $\sim 2.9 \mathrm{~V}$, appropriate for the phosphorene based Li-ion battery. Similar to the Li-diffusion on phosphorene, the Na diffusion is anisotropic and its value along the zig-zag direction is predicted to be $\sim 0.04 \mathrm{eV} .{ }^{344,345}$ Experimental works concerning phosphorene as the anode for $\mathrm{Li}$ and $\mathrm{Na}$ ion batteries have also been presented. ${ }^{346-348}$ Sun et al. experimentally studied fewlayer phosphorene sandwiched between graphene layers used as a high-capacity anode for $\mathrm{Na}$ ion batteries. ${ }^{347}$ They found that the hybrid phosphorene- graphene material possesses a specific capacity of $2440 \mathrm{~mA} \mathrm{~h} \mathrm{~g}^{1}$ at a current density of $0.05 \mathrm{~A} \mathrm{~g}^{1}$ and $83 \%$ capacity retention after 100 cycles while operating between 0 and $1.5 \mathrm{~V}$. To explain the large capacity of the anode material, they used in situ transmission electron microscopy and ex situ X-ray diffraction techniques, and found a dual mechanism of intercalation of Na-ions along the zig-zag direction of phosphorene followed by the formation of a $\mathrm{Na}_{3} \mathrm{P}$ alloy. The superior electrochemical properties of the sandwiched hybrid structure could render it a suitable anode material for sodium-ion batteries.

Metallic buckled antimonene nanosheets-graphene (SbNS-G) films have been synthesized to present high volumetric capacity, high-rate capability and good cycle performance for sodium storage. ${ }^{243}$ In the initial cycle at a current density of $0.1 \mathrm{~mA} \mathrm{~cm}^{-2}$, the reversible volumetric capacity reached a very high value of $1226 \mathrm{~mA} \mathrm{~h} \mathrm{~cm}^{-3}$ for the SbNS-G film with a mass loading of $1.6 \mathrm{mg} \mathrm{cm}^{-2}$. After 50 cycles, the reversible volumetric capacity is almost stable at $650 \mathrm{~mA} \mathrm{~h} \mathrm{~cm}{ }^{-3}$. It is worth noting that the good flexibility of graphene relieves the stress of the notorious volume change of metallic $\mathrm{Sb}$. The research effectively illustrates that the metallic Sb nanosheets will have broad applications in the areas of $\mathrm{Na}$ ion batteries.

Owing to the puckered and buckled structural properties, 2D group-VA materials have low atomic packing factors, indicating that these elements are favorable for accommodation of $\mathrm{Li}$ and $\mathrm{Na}$ atoms. Besides, the rich alloy phases $\mathrm{Li}_{3} \mathrm{VA}$ and $\mathrm{Na}_{3} \mathrm{VA}$ (VA is the group element: $\mathrm{P}, \mathrm{As}$, $\mathrm{Sb}$ and $\mathrm{Bi}$ ) provide their high capacities for Li-ion (Na-ion) batteries. Specifically, the high surface areas of group-VA nanosheets will give those higher capacities and faster ion diffusion as anodes in $\mathrm{Li}$ and $\mathrm{Na}$ ion batteries. 


\subsection{Thermoelectric}

A thermoelectric material is a kind of promising material that can convert waste heat directly into electrical energy (Fig. 22d). The performance of thermoelectric materials can be quantified by a dimensionless figure of merit ZT: ZT $=\mathrm{S}^{2} \mathrm{sT} / \mathrm{k}$, where $\mathrm{S}$ is the Seebeck coefficient, $\mathrm{s}$ is the electrical conductivity, $\mathrm{T}$ is the temperature, and $\mathrm{k}=\mathrm{k}_{\mathrm{e}}+\mathrm{k}_{\mathrm{p}}$ is the total thermal conductance that is usually split into electron $\left(\mathrm{k}_{\mathrm{e}}\right)$ and phonon $\left(\mathrm{k}_{\mathrm{p}}\right)$ contributions. ${ }^{349}$ In order to make materials suitable for thermoelectric generation, it is desirable to have a ZT larger than three. ${ }^{350}$ Unfortunately, as described by the Wiedemann- Franz law, the electrical conductivity is proportional to the thermal conductivity and it becomes impossible to increase electrical conductivity and suppress thermal conductivity simultaneously. Compared with bulk crystals, low dimensional materials can escape from the Wiedemann-Franz law and have the potential to achieve higher ZT by different engineering approaches. ${ }^{351,352}$

Theoretical works indicate that 2D group-VA materials have great potential in thermoelectric applications. ${ }^{137,138,353-356}$ Due to the anisotropic puckered structures, group-VA crystals exhibit strong anisotropy in electrical and thermal conductivities, where the value of ZT along the armchair direction is much larger than that along the zig-zag direction. ${ }^{355,357-359}$ Fei et al. predicted that depending on the $\mathrm{p}$ or $\mathrm{n}$ doping and orthogonal electric field, the thermoelectric performance of phosphorene can be strongly enhanced. Its figure of merit ZT can reach $\sim 1.5$ along the armchair direction at room temperature. ${ }^{139}$ Additionally, some groups studied the effect of the applied strain on the thermoelectric effect of black phosphorus. ${ }^{21,353,356}$ When the zig-zag direction strain is applied, the Seebeck coefficient and electrical conductivity in the zig-zag direction can be greatly enhanced at a strain of $5 \%$. When the armchair direction strain of $8 \%$ is applied, the room temperature ZT value can reach 2.12 in the armchair direction of phosphorene. ${ }^{356}$ By nano-structure engineering (cutting a monolayer phosphorene along armchair or zigzag directions), Zhang et al. showed that very good thermoelectric performance of phosphorene can be obtained. ${ }^{23}$ The ZT value of phosphorene nanoribbons with armchair edges can be optimized to as high as 6.4 at room temperature, which suggests that phosphorene nanoribbons could be very promising candidates for high-performance thermoelectric applications.

Promisingly, Sandonas et al. explored the thermoelectric performance of the puckered phosphorene and arsenene. Puckered arsenene exhibits a stronger anisotropic thermoelectric response than phosphorene. ${ }^{355}$ At $300 \mathrm{~K}$ with moderate $\mathrm{n}$ type doping, the ZT value of arsenene can reach $~ 1.0$ along the armchair direction. For antimonene, its thermoelectric properties have not been reported and only its thermal conductivity s has been studied. Zhang et al. found that buckled antimonene exhibits a relatively low thermal conductivity. ${ }^{137}$ Furthermore, chemical functionalization can lower this value even further, indicating that antimonene is a potential excellent thermoelectric material. Considering the vast amount of research studies, phosphorene, arsenene and antimonene have great potential to be good thermoelectric materials by engineering approaches.

Besides, Cheng et al. evaluated the figure of merit ZT of buckled and puckered bismuthene at different temperatures. ${ }^{128,138}$ Buckled bismuthene exhibits much larger ZT than its bulk structure as indicated by first-principles calculations combined with the Boltzmann transport equation. ${ }^{128}$ The better thermoelectric performance is attributed to the fact that buckled bismuthene exhibits very large power factors $\left(S^{2} s\right)$ and lower thermal conductivity. The ZT value of buckled bismuthene can be optimized to as high as 2.4 at room temperature and it can be further improved to 4.1 at $500 \mathrm{~K}$. Their theoretical works predicted that puckered bismuthene exhibits a very high ZT value of 6.4 for n-type systems at room temperature. ${ }^{138}$ This value significantly exceeds that of buckled bismuthene (2.4). ${ }^{128}$ Moreover, it is demonstrated that the ZT values are all higher than 2.0 in relatively broad regions of both temperature and carrier concentration. The obviously improved thermoelectric performance of puckered bismuthene is inherently related to the weak electron-phonon coupling strength, as identified by the very small deformation potential constant. 
Considering the rapid progress of fabrication techniques, it is reasonable to expect that high thermoelectric performance could be realized in bismuthene.

\section{Summary and outlook}

The initial progress in 2D phosphorene, arsenene, antimonene and bismuthene has motivated a revisit of group-VA materials, with a focus on accelerating the discovery of materials that complement the properties of their bulk van der Waals solids. In particular, unlike semimetallic group-IVA (graphene, silicene, germanene and stanene) and metallic group-IIIA (borophene) materials, 2D group-VA materials are semiconducting, with a broad range of band gaps, from 0.36 to $2.62 \mathrm{eV}$, providing unprecedented opportunities for device applications. This review article presents a theoretical and experimental overview of the state-of-the-art research in the 2D group-VA material family, in terms of their structural allotropes, basic fundamental properties, exotic electronic properties, material preparation methods, and various possible applications.

Phosphorene, among the 2D group-VA family members, is the first and most studied member. It presents fascinating properties, such as the highly tunable direct bandgap, high carrier mobility, negative Poisson's ratio, and predominantly anisotropic electronic and optical properties. Importantly, these properties can be further tuned by various external factors, e.g., doping, defect engineering, strain, electric field, heterostructure and chemical functionalization, etc. In particular, compared with graphene, $\mathrm{MoS}_{2}$ and other 2D materials, black phosphorene exhibits more prominent application advantages in electronic devices and optoelectronic devices. However, one Achilles' heel of phosphorene is the presence of its intrinsic instability due to its fast oxidation behavior coupled with light-induced degradation of exfoliated flakes under air moisture conditions. Several methods have been developed to solve this issue, and the stability mechanisms are being explored. However, the practical application of phosphorene is still in its infancy. Thus, to accelerate the reality of phosphorene devices, researchers have to focus on mitigating its oxidation issue and enhancing its long-term stability under ambient conditions.

Regarding arsenene, more emerging reports are based on first-principles DFT calculations. Experimentally, in the process of preparing 2D arsenene, highly toxic arsenic trioxide can be produced, which hinders the experimental exploration of arsenene. Nevertheless, impressively, a plasma-assisted process has been used to synthesize multilayer arsenene on InAs. In addition, the aqueous shear exfoliation method has also been used to fabricate arsenene nanosheets for electrochemical applications. These works provide experimental evidence for the successful synthesis of 2D arsenene and may pave the way for further experimental investigations of the unique properties of arsenene.

In terms of antimonene, several theoretical works have predicted a variety of exotic properties and applications ranging from optoelectronics to energy harvesting or unusual topological features originated from its electronic band structure. Since 2016, a number of experimental results have made all those expectations somewhat closer to reality. In particular, antimonene was isolated for the first time demonstrating environmental stability. We expect that the potential applications of antimonene and related materials will be expanded in the near future to cover medicine, catalysis, etc.

Bismuthene has received widespread attention due to its quantum size effects, long mean free path, low carrier density, large spin-orbit coupling and so on. In particular, the realization of a bismuthene based 2D topological insulator will be of great importance in monoelement materials. Stable bismuth 
films have been fabricated by MBE and aqueous shear exfoliation methods. Interestingly, monolayer bismuthene has also been grown on a $\mathrm{SiC}(0001)$ substrate, and the substrate not only stabilizes the quasi2D topological insulator but also plays a pivotal role in achieving the large gap. Excitingly, the bulk gap is up to $0.8 \mathrm{eV}$, and thus bismuthene can be regarded as a candidate for a high-temperature quantum spin Hall material. The composite method incorporating monolayer-substrate by using other group-VA monolayer arsenene and antimonene may open a systematic route to generate large-gap quantum spin Hall systems.

It should be noted that one of the most charming properties of phosphorene, arsenene, antimonene and bismuthene is their colorful allotropes, which distinguishes them from other 2D materials. For instance, black phosphorene in the puckered form is the most prominent allotrope, and other allotropes are metastable phases, such as the recent successfully fabricated blue phosphorene. The buckled $b$ phases are the lowest-energy configuration, which are different from the most stable black phosphorene structure. Many allotropes of arsenene, antimonene and bismuthene can also be obtained. Furthermore, three allotropes, namely a, b and $\mathrm{z}$, found in bismuthene have comparable average binding energy. These 2D group-VA monolayer allotropes with different band alignments can facilitate the designing of 2D material based van der Waals heterostructures, which are of utmost importance for novel electronic and optoelectronic applications.

Despite the excellent intrinsic properties and great variety of $2 \mathrm{D}$ group-VA materials, the main challenge is the lack of an environment-friendly and low-cost synthesis method for large-scale production. High-quality, large-area growth should be developed to facilitate more powerful functional devices such as field effect transistors, photodetectors, light-emitting devices, topological spintronic devices, gas sensors, lithium and sodium batteries, and thermoelectric devices. The unique properties and wide variety of allotropes of 2D group-VA materials are also favourable for practical applications in terms of biosensors, medical treatments, non-linear optics, catalysis, thermophotovoltaic cells, etc. ${ }^{80-}$ ${ }^{82,360,361}$ The 2D group-VA materials, from phosphorene, arsenene, antimonene, to bismuthene, offer a novel platform to study new emerging device physics in these atomically thin 2D systems and create new concepts for nano-devices. This review article aims at establishing a well-defined impression of 2D group-VA materials, from their atomic structures, fundamental properties, electronic modulations, synthesis methods, to various potential applications. We hope that this review will inspire more exciting discoveries and applications in this growing family of 2D group-VA materials.

\section{Conflicts of interest}

There are no conflicts to declare.

\section{Acknowledgements}

This work was financially supported by the National Basic Research Program of China (2014CB931702), National Key Research \& Development Projects of China (2016YFA0202301), NSFC (51572128, 21403109, 61725107, 51572290), NSFC-RGC (5151101197), the Natural Science Foundation of Jiangsu Province (BK20140769), the National Science Foundation for Distinguished Young Scholars of China (61725402), the Fundamental Research Funds for the Central Universities (No. 30916015106), and PAPD of Jiangsu Higher Education Institutions, and in USA by DoD (Grant W911NF-15-1-0650), in 
Spain the Spanish Ministerio de Economı'a y Competitividad through the "Marı'a de Maeztu" Programme for Units of Excellence in R\&D (MDM-2014-0377) and the projects MAT2016-77608-C3-1$\mathrm{P}$ and $-\mathrm{C} 3-3-\mathrm{P}$.

\section{References}

1 F. Xia, H. Wang, D. Xiao, M. Dubey and A. Ramasubramaniam, Nat. Photonics, 2014, 8, 899-907.

2 D. Franklin, Science, 2015, 349, aab2750.

3 P. Miro, M. Audi $\square$ red and T. Heine, Chem. Soc. Rev., 2014, 43, 6537-6554.

4 H. Zeng and X. Cui, Chem. Soc. Rev., 2015, 44, 2629-2642.

5 Y. Guo, K. Xu, C. Wu, J. Zhao and Y. Xie, Chem. Soc. Rev., 2015, 44, 637-646.

6 H. Liu, Y. Du, Y. Deng and P. D. Ye, Chem. Soc. Rev., 2015, 44, 2732-2743.

7 M. Chhowalla, D. Jena and H. Zhang, Nat. Rev. Mater., 2016, 1, 16052.

8 C. Tan, X. Cao, X. J. Wu, Q. He, J. Yang, X. Zhang, J. Chen, W. Zhao, S. Han, G. H. Nam, M. Sindoro and H. Zhang, Chem. Rev., 2017, 117, 6225-6331.

9 X. Kong, Q. Liu, C. Zhang, Z. Peng and Q. Chen, Chem. Soc. Rev., 2017, 46, 2127-2157.

10 M. Akhtar, G. Anderson, R. Zhao, A. Alruqi, J. E. Mroczkowska, G. Sumanasekera and J. B. Jasinski, npj 2D Mater. Appl., 2017, 1, 5.

11 B. Anasori, M. R. Lukatskaya and Y. Gogotsi, Nat. Rev. Mater., 2017, 2, 16098.

12 W. Lei, G. Liu, J. Zhang and M. Liu, Chem. Soc. Rev., 2017, 46, 3492-3509.

13 D. Akinwande, N. Petrone and J. Hone, Nat. Commun., 2014, 5, 5678.

14 R. Fei and L. Yang, Nano Lett., 2014, 14, 2884-2889.

15 J. Qiao, X. Kong, Z. X. Hu, F. Yang and W. Ji, Nat. Commun., 2014, 5, 4475.

16 L. Li, Y. Yu, G. J. Ye, Q. Ge, X. Ou, H. Wu, D. Feng, X. H. Chen and Y. Zhang, Nat. Nanotechnol., 2014, 9, 372377.

17 Q. Liu, X. Zhang, L. B. Abdalla, A. Fazzio and A. Zunger, Nano Lett., 2015, 15, 1222-1228.

18 F. Xia, H. Wang and Y. Jia, Nat. Commun., 2014, 5, 4458.

19 V. Tran, R. Soklaski, Y. Liang and L. Yang, Phys. Rev. B: Condens. Matter Mater. Phys., 2014, 89, 235319.

20 T. Low, A. S. Rodin, A. Carvalho, Y. Jiang, H. Wang, F. Xia and A. H. Castro Neto, Phys. Rev. B: Condens. Matter Mater. Phys., 2014, 90, 075434.

21 G. Qin, Q. B. Yan, Z. Qin, S. Y. Yue, H. J. Cui, Q. R. Zheng and G. Su, Sci. Rep., 2014, 4, 6946.

22 M. Pumera and Z. Sofer, Adv. Mater., 2017, 29, 1605299.

23 J. Zhang, H. J. Liu, L. Cheng, J. Wei, J. H. Liang, D. D. Fan, J. Shi, X. F. Tang and Q. J. Zhang, Sci. Rep., 2014, 4, 6452.

24 J. W. Jiang and H. S. Park, Nat. Commun., 2014, 5, 4727.

25 H. Y. Zhang and J. W. Jiang, J. Phys. D: Appl. Phys., 2015, 48, 455305.

26 S. Zhang, Z. Yan, Y. Li, Z. Chen and H. Zeng, Angew. Chem., Int. Ed., 2015, 54, 3112-3115.

27 S. Zhang, M. Xie, F. Li, Z. Yan, Y. Li, E. Kan, W. Liu, Z. Chen and H. Zeng, Angew. Chem., 2016, 128, 1698-1701.

28 G. Wang, R. Pandey and S. P. Karna, ACS Appl. Mater. Interfaces, 2015, 7, 11490-11496.

29 H. Tsai, S. Wang, C. Hsiao, C. Chen, H. Ouyang, Y. Chueh,

H. Kuo and J. Liang, Chem. Mater., 2016, 28, 425-429.

30 H. S. Tsai, C. W. Chen, C. H. Hsiao, H. Ouyang and J. H. Liang, Chem. Commun., 2016, 52, 8409-8412.

31 P. Ares, F. Aguilar-Galindo, D. Rodriguez-San-Miguel, D. A. Aldave, S. Diaz-Tendero, M. Alcami, F. Martin, J. Gomez-Herrero and F. Zamora, Adv. Mater., 2016, 28, 6332-6336.

32 C. Gibaja, D. Rodriguez-San-Miguel, P. Ares, J. Gomez-Herrero, M. Varela, R. Gillen, J. Maultzsch, F. Hauke, A. Hirsch, G. Abellan and F. Zamora, Angew. Chem., Int. Ed., 2016, 55, 14345-14349.

33 J. Ji, X. Song, J. Liu, Z. Yan, C. Huo, S. Zhang, M. Su, L. Liao, W. Wang, Z. Ni, Y. Hao and H. Zeng, Nat. Commun., 2016, 7, 13352.

34 X. Wu, Y. Shao, H. Liu, Z. Feng, Y. L. Wang, J. T. Sun, C. Liu,J. O. Wang, Z. L. Liu, S. Y. Zhu, Y. Q. Wang, S. X. Du, Y. G. Shi, J. Ibrahim and H. J. Gao, Adv. Mater., 2017, 29, 1605407.

35 P. Ares, F. Zamora and J. Gomez-Herrero, ACS Photonics, 2017, 4, 600-605.

36 G. Pizzi, M. Gibertini, E. Dib, N. Marzari, G. Iannaccone and G. Fiori, Nat. Commun., 2016, 7, 12585.

37 C. Kamal and M. Ezawa, Phys. Rev. B: Condens. Matter Phys., 2015, 91, 085423 
38 O. U. Akturk, V. O. Ozçelik and S. Ciraci, Phys. Rev. B: Condens. Matter Mater. Phys., 2015, 91, 235446.

39 O. Uzengi Aktu“rk, E. Aktu"rk and S. Ciraci, Phys. Rev. B: Condens. Matter Mater. Phys., 2016, 93, 035450.

40 M. Zhao, X. Zhang and L. Li, Sci. Rep., 2015, 5, 16108.

41 J. Zhou, Q. Sun, Q. Wang, Y. Kawazoe and P. Jena, Nanoscale, 2016, 8, 11202-11209.

42 Q. Sun, Y. Dai, Y. Ma, N. Yin, W. Wei, L. Yu and B. Huang, 2D Mater., 2016, 3, 035017.

43 D. Singh, S. K. Gupta, Y. Sonvane and I. Lukac`evic', J. Mater. Chem. C, 2016, 4, 6386-6390.

44 L. Kou, Y. Ma, X. Tan, T. Frauenheim, A. Du and S. Smith, J. Phys. Chem. C, 2015, 119, 6918-6922.

45 Z. Zhu, J. Guan and D. Toma'nek, Phys. Rev. B: Condens. Matter Mater. Phys., 2015, 91, 161404.

46 S. Zhang, Y. Hu, Z. Hu, B. Cai and H. Zeng, Appl. Phys. Lett., 2015, 107, 022102.

47 H. Zhang, Y. Ma and Z. Chen, Nanoscale, 2015, 7, 19152-19159.

48 M. Zeraati, S. M. Vaez Allaei, I. Abdolhosseini Sarsari, M. Pourfath and D. Donadio, Phys. Rev. B: Condens. Matter Mater. Phys., 2016, 93, 085424.

49 Y. Nie, M. Rahman, D. Wang, C. Wang and G. Guo, Sci. Rep., 2015, 5, 17980.

50 V. O. Ozçelik, O. U. Akturk, E. Durgun and S. Ciraci, Phys. Rev. B: Condens. Matter Mater. Phys., 2015, 92, 125420.

51 J. Lee, W. C. Tian, W. L. Wang and D. X. Yao, Sci. Rep., 2015, 5, 11512-11527.

52 Q. Zhang and U. Schwingenschlo“gl, Phys. Rev. B: Condens. Matter Mater. Phys., 2016, 93, 045312.

53 Y. P. Wang, W. X. Ji, C. W. Zhang, P. Li, F. Li, M. J. Ren, X. L. Chen, M. Yuan and P. J. Wang, Sci. Rep., 2016, 6, 20342.

54 J. Zhao, Y. Li and J. Ma, Nanoscale, 2016, 8, 9657-9666.

55 D. Wang, L. Chen, C. Shi, X. Wang, G. Cui, P. Zhang and Y. Chen, Sci. Rep., 2016, 6, 28487.

56 Y. Lu, D. Zhou, G. Chang, S. Guan, W. Chen, Y. Jiang, J. Jiang, X.-s. Wang, S. A. Yang, Y. P. Feng, Y. Kawazoe and H. Lin, npj Comput. Mater., 2016, 2, 16011.

57 M. Y. Liu, Y. Huang, Q. Y. Chen, C. Cao and Y. He, Sci. Rep., 2016, 6, 29114.

58 S. Zhang, M. Xie, B. Cai, H. Zhang, Y. Ma, Z. Chen, Z. Zhu, Z. Hu and H. Zeng, Phys. Rev. B: Condens. Matter Mater. Phys., 2016, 93, 245303.

59 J. Zhao, W. Guo and J. Ma, Nano Res., 2016, 10, 491-502.

60 J. Zhao, C. Liu, W. Guo and J. Ma, Nanoscale, 2017, 9, 7006-7011.

61 B. Fu, W. Feng, X. Zhou and Y. Yao, 2D Mater., 2017, 4, 025107.

62 X. Chen, L. Wang, X. Sun, R. Meng, J. Xiao, H. Ye and G. Zhang, IEEE Electron Device Lett., 2017, 38, 661-664.

63 S. Zhang, W. Zhou, Y. Ma, J. Ji, B. Cai, S. A. Yang, Z. Zhu, Z. Chen and H. Zeng, Nano Lett., 2017, 17, 3434-3440.

64 H. Sevincli, Nano Lett., 2017, 17, 2589-2595.

65 B. Peng, D. Zhang, H. Zhang, H. Shao, G. Ni, Y. Zhu and H. Zhu, Nanoscale, 2017, 9, 7397-7407.

66 Y. Wang, P. Huang, M. Ye, R. Quhe, Y. Pan, H. Zhang, H. Zhong, J. Shi and J. Lu, Chem. Mater., 2017, 29, 21912201.

67 Q. Sun, Y. Dai, C. Niu, Y. Ma, W. Wei, L. Yu and B. Huang, 2D Mater., 2017, 4, 025038.

68 A. N. Rudenko, M. I. Katsnelson and R. Rolda'n, Phys. Rev. B: Condens. Matter Mater. Phys., 2017, 95, 081407.

69 R. Meng, M. Cai, J. Jiang, Q. Liang, X. Sun, Q. Yang, C. Tan and X. Chen, IEEE Electron Device Lett., 2017, 38, 134-137.

70 F. F. Zhu, W. J. Chen, Y. Xu, C. L. Gao, D. D. Guan, C. H. Liu, D. Qian, S. C. Zhang and J. F. Jia, Nat. Mater., $2015,14,1020-1025$.

71 J. Zhao, H. Liu, Z. Yu, R. Quhe, S. Zhou, Y. Wang, C. C. Liu, H. Zhong, N. Han, J. Lu, Y. Yao and K. Wu, Prog. Mater. Sci., 2016, 83, 24-151.

72 Z. Ni, Q. Liu, K. Tang, J. Zheng, J. Zhou, R. Qin, Z. Gao, D. Yu and J. Lu, Nano Lett., 2012, 12, 113-118.

73 E. Bianco, S. Butler, S. Jiang, O. D. Restrepo, W. Windl and J. E. Goldberger, ACS Nano, 2013, 7, 4414-4421.

74 L. Tao, E. Cinquanta, D. Chiappe, C. Grazianetti, M. Fanciulli, M. Dubey, A. Molle and D. Akinwande, Nat. Nanotechnol., 2015, 10, 227-231.

75 A. J. Mannix, X.-F. Zhou, B. Kiraly, J. D. Wood, D. Alducin, B. D. Myers, X. Liu, B. L. Fisher, U. Santiago, J. R. Guest, M. J. Yacaman, A. Ponce, A. R. Oganov, M. C. Hersam and M. P. Guisinger, Science, 2015, 350, 1513-1516.

76 G. Tai, T. Hu, Y. Zhou, X. Wang, J. Kong, T. Zeng, Y. You and Q. Wang, Angew. Chem., 2015, 127, 15693-15697.

77 B. Feng, J. Zhang, Q. Zhong, W. Li, S. Li, H. Li, P. Cheng, S. Meng, L. Chen and K. Wu, Nat. Chem., 2016, 8, 563568.

78 B. Feng, J. Zhang, R. Liu, T. Iimori, C. Lian, H. Li, L. Chen, K. Wu, S. Meng, F. Komori and I. Matsuda, Phys. Rev. B: Condens. Matter Mater. Phys., 2016, 94, 041408. 
79 M. Moreno-Moreno, G. Lopez-Polin, A. Castellanos-Gomez, C. Gomez-Navarro and J. Gomez-Herrero, 2D Mater., 2016, 3, 031007.

80 R. Gusmao, Z. Sofer, D. Bousa and M. Pumera, Angew. Chem., Int. Ed., 2017, 56, 14417-14422.

81 L. Lu, X. Tang, R. Cao, L. Wu, Z. Li, G. Jing, B. Dong, S. Lu, Y. Li, Y. Xiang, J. Li, D. Fan and H. Zhang, Adv. Opt. Mater., 2017, 5, 1700301.

82 J. Zhang, F. Li, M. Xue, J. Li, X. Ma, L. Chen, X. Zhang and

D. MacFarlane, Angew. Chem., Int. Ed., 2017, 56, 14718-14722.

83 F. Reis, G. Li, L. Dudy, M. Bauernfeind, S. Glass, W. Hanke, R. Thomale, J. Scha“fer and R. Claessen, Science, 2017, 357, 287-290.

84 N. Hussain, T. Liang, Q. Zhang, T. Anwar, Y. Huang, J. Lang, K. Huang and H. Wu, Small, 2017, 13, 1701349.

85 L. Lu, Z. Liang, L. Wu, Y. Chen, Y. Song, S. C. Dhanabalan, J. S. Ponraj, B. Dong, Y. Xiang, F. Xing, D. Fan and H. Zhang, Laser Photonics Rev., 2017, DOI: 10.1002/lpor.201700221.

86 S. Balendhran, S. Walia, H. Nili, S. Sriram and M. Bhaskaran, Small, 2015, 11, 640-652.

87 A. Ambrosi, C. K. Chua, N. M. Lati $\square$, A. H. Loo, C. H. Wong,A. Y. Eng, A. Bonanni and M. Pumera, Chem. Soc. Rev., 2016, 45, 2458-2493.

88 Y. Jing, X. Zhang and Z. Zhou, Wiley Interdiscip. Rev.: Comput. Mol. Sci., 2016, 6, 5-19.

89 R. Gusmao, Z. Sofer and M. Pumera, Angew. Chem., Int. Ed., 2017, 129, 8164-8185.

90 A. J. Mannix, B. Kiraly, M. C. Hersam and N. P. Guisinger, Nat. Rev. Chem., 2017, 1, 0014.

91 A. Molle, J. Goldberger, M. Houssa, Y. Xu, S. C. Zhang and D. Akinwande, Nat. Mater., 2017, 16, 163-169.

92 P. Chen, N. Li, X. Chen, W. Ong and X. Zhao, 2D Mater., 2017, 5, 014002.

93 M. Jamali, J. S. Lee, J. S. Jeong, F. Mahfouzi, Y. Lv, Z. Zhao, B. K. Nikolic, K. A. Mkhoyan, N. Samarth and J. P. Wang, Nano Lett., 2015, 15, 7126-7132.

94 R. Hultgren, N. S. Gingrich and B. E. Warren, J. Chem. Phys., 1935, 3, 351-355.

95 A. Brown and S. Rundqvist, Acta Crystallogr., 1965, 19, 684-685.

96 H. Thurn and H. Kerbs, Angew. Chem., Int. Ed. Engl., 1966, 5, 1047-1048.

97 R. W. Keyes, Phys. Rev., 1953, 92, 580-584.

98 D. Warschauer, J. Appl. Phys., 1963, 34, 1853-1860.

99 J. C. Jamieson, Science, 1963, 139, 1291-1292.

100 P. W. Bridgman, J. Am. Chem. Soc., 1914, 36, 1344-1363.

101 P. W. Bridgman, J. Am. Chem. Soc., 1916, 38, 609-612.

102 P. W. Bridgman, Phys. Rev., 1914, 3, 489-490.

103 L. Q. Sun, M. J. Li, K. Sun, S. H. Yu, R. S. Wang and H. M. Xie, J. Phys. Chem. C, 2012, 116, 14772-14779.

104 S. S. Boksha, J. Cryst. Growth, 1968, 3, 426-429.

105 M. Köpf, N. Eckstein, D. Pfister, C. Grotz, I. Krüger, M. Greiwe, T. Hansen, H. Kohlmann and T. Nilges, J. Cryst. Growth, 2014, 405, 6-10.

106 T. Nilges, M. Kersting and T. Pfeifer, J. Solid State Chem., 2008, 181, 1707-1711.

107 B. Mamoru, I. Fukunori, T. Yuji and M. Akira, Jpn. J. Appl. Phys., 1989, $28,1019$.

108 N. C. Norman, Chemistry of arsenic, antimony and bismuth, Springer Science \& Business Media, 1997.

109 J. H. Xu, E. G. Wang, C. S. Ting and W. P. Su, Phys. Rev. B: Condens. Matter Mater. Phys., 1993, 48, 17271.

110 H. Krebs, W. Holz and K. H. Worms, Eur. J. Inorg. Chem., 1957, 1031-1037.

111 G. N. Greaves, S. R. Elliott and E. A. Davis, Adv. Phys., 1979, 28, 49-141.

112 H. Liu, A. T. Neal, Z. Zhu, X. Xu, D. Tomanek, P. D. Ye and Z. Luo, ACS Nano, 2014, 8, 4033-4041.

113 Z. Zhu and D. Tomanek, Phys. Rev. Lett., 2014, 112, 176802.

114 M. Wu, H. Fu, L. Zhou, K. Yao and X. C. Zeng, Nano Lett., 2015, 15, 3557-3562.

115 Z. Zhuo, X. Wu and J. Yang, J. Am. Chem. Soc., 2016, 138, 7091-7098.

116 J. P. Perdew, K. Burke and M. Ernzerhof, Phys. Rev. Lett., 1996, 77, 3865.

117 J. Heyd, G. E. Scuseria and M. Ernzerhof, J. Chem. Phys., 2006, 124, 219906.

118 L. Hedin, Phys. Rev., 1965, 139, A796-A823.

119 M. Shishkin and G. Kresse, Phys. Rev. B: Condens. Matter Mater. Phys., 2006, 74, 035101.

120 Y. Cai, G. Zhang and Y. W. Zhang, Sci. Rep., 2014, 4, 6677.

121 X. Wang, A. M. Jones, K. L. Seyler, V. Tran, Y. Jia, H. Zhao, H. Wang, L. Yang, X. Xu and F. Xia, Nat. Nanotechnol., 2015, 10, 517-521.

122 L. Liang, J. Wang, W. Lin, B. G. Sumpter, V. Meunier and M. Pan, Nano Lett., 2014, 14, 6400-6406.

123 J. H. Choi, P. Cui, H. Lan and Z. Zhang, Phys. Rev. Lett., 2015, 115, 066403. 
124 J. L. Zhang, S. Zhao, C. Han, Z. Wang, S. Zhong, S. Sun, R. Guo, X. Zhou, C. D. Gu, K. D. Yuan, Z. Li and W. Chen, Nano Lett., 2016, 16, 4903-4908.

125 F. Ersan, E. Aktü.rk and S. Ciraci, Phys. Rev. B: Condens. Matter Mater. Phys., 2016, 94, 245417.

126 E. Aktu rk, O. U . Aktu rk and S. Ciraci, Phys. Rev. B: Condens. Matter Mater. Phys., 2016, 94, 014115.

127 R. R. Q. Freitas, R. Rivelino, F. de Brito Mota, C. M. C. de Castilho, A. Kakanakova-Georgieva and G. K. Gueorguiev, J. Phys. Chem. C, 2015, 119, 23599-23606.

128 L. Cheng, H. Liu, X. Tan, J. Zhang, J. Wei, H. Lv, J. Shi and X. Tang, J. Phys. Chem. C, 2014, 118, 904-910.

129 Y. Cai, G. Zhang and Y. W. Zhang, J. Am. Chem. Soc., 2014, 136, 6269-6275.

130 Z. Zhang, J. Xie, D. Yang, Y. Wang, M. Si and D. Xue, Appl. Phys. Express, 2015, 8, 055201.

131 Y. Aierken, D. Çakır, C. Sevik and F. M. Peeters, Phys. Rev. B: Condens. Matter Mater. Phys., 2015, 92, 081408

132 Y. Cai, Q. Ke, G. Zhang, Y. P. Feng, V. B. Shenoy and Y. W. Zhang, Adv. Funct. Mater., 2015, 25, 2230-2236.

133 Z. Ong, Y. Cai, G. Zhang and Y. Zhang, J. Phys. Chem. C, 2014, 118, 25272-25277.

134 G. Qin, Q. B. Yan, Z. Qin, S. Y. Yue, M. Hu and G. Su, Phys. Chem. Chem. Phys., 2015, 17, 4854-4858.

135 L. Zhu, G. Zhang and B. Li, Phys. Rev. B: Condens. Matter Mater. Phys., 2014, 90, 214302.

136 S. Wang, W. Wang and G. Zhao, Phys. Chem. Chem. Phys., 2016, 18, 31217-31222.

137 T. Zhang, Y. Y. Qi, X. R. Chen and L. C. Cai, Phys. Chem. Chem. Phys., 2016, 18, 30061-30067.

138 L. Cheng, H. J. Liu, J. Zhang, J. Wei, J. H. Liang, P. H. Jiang, D. D. Fan, L. Sun and J. Shi, Phys. Chem. Chem. Phys., 2016, 18, 17373-17379.

139 R. Fei, A. Faghaninia, R. Soklaski, J. A. Yan, C. Lo and L. Yang, Nano Lett., 2014, 14, 6393-6399.

140 Q. Wei and X. Peng, Appl. Phys. Lett., 2014, 104, 251915.

141 L. Wang, A. Kutana, X. Zou and B. I. Yakobson, Nanoscale, 2015, 7, 9746-9751.

142 D. Kecik, E. Durgun and S. Ciraci, Phys. Rev. B: Condens. Matter Mater. Phys., 2016, 94, 205409.

143 A. S. Rodin, A. Carvalho and A. H. Castro Neto, Phys. Rev. Lett., 2014, 112, 176801.

144 X. Peng, Q. Wei and A. Copple, Phys. Rev. B: Condens. Matter Mater. Phys., 2014, 90, 085402.

145 J. Dai and X. C. Zeng, J. Phys. Chem. Lett., 2014, 5, 1289-1293.

146 Y. Liu, F. Xu, Z. Zhang, E. S. Penev and B. I. Yakobson, Nano Lett., 2014, 14, 6782-6786.

147 W. Hu and J. Yang, J. Phys. Chem. C, 2015, 119, 20474-20480.

148 M. Umar Farooq, A. Hashmi and J. Hong, Sci. Rep., 2015, 5, 12482.

149 T. Hu and J. Dong, Nanotechnology, 2015, 26, 065705.

150 Y. Guo and J. Robertson, Sci. Rep., 2015, 5, 14165.

151 P. Srivastava, K. P. S. S. Hembram, H. Mizuseki, K. R. Lee, S. S. Han and S. Kim, J. Phys. Chem. C, 2015, 119, 6530-6538.

152 K. Iordanidou, J. Kioseoglou, V. V. Afanas'ev, A. Stesmans andM. Houssa, Phys. Chem. Chem. Phys., 2017, 19, $9862-$ 9871.

153 X. Sun, Y. Liu, Z. Song, Y. Li, W. Wang, H. Lin, L. Wang and Y. Li, J. Mater. Chem. C, 2017, 5, 4159-4166.

154 L. Yang, Y. Song, W. Mi and X. Wang, RSC Adv., 2016, 6, 66140-66146.

155 G. Wang, R. Pandey and S. P. Karna, Appl. Phys. Lett., 2015, 106, 173104.

156 Y. Jing, Q. Tang, P. He, Z. Zhou and P. Shen, Nanotechnology, 2015, 26, 095201.

157 Y. Cai, Q. Ke, G. Zhang and Y. W. Zhang, J. Phys. Chem. C, 2015, 119, 3102-3110.

158 R. Zhang, B. Li and J. Yang, J. Phys. Chem. C, 2015, 119, 2871-2878.

159 L. Kou, T. Frauenheim and C. Chen, J. Phys. Chem. Lett., 2014, 5, 2675-2681.

160 T. Hu and J. Hong, J. Phys. Chem. C, 2015, 119, 8199-8207.

161 V. V. Kulish, O. I. Malyi, C. Persson and P. Wu, Phys. Chem. Chem. Phys., 2015, 17, 992-1000.

162 X. Sui, C. Si, B. Shao, X. Zou, J. Wu, B. L. Gu and W. Duan, J. Phys. Chem. C, 2015, 119, 10059-10063.

163 L. Seixas, A. Carvalho and A. H. Castro Neto, Phys. Rev. B: Condens. Matter Mater. Phys., 2015, 91, 155138.

164 V. Wang, Y. Kawazoe and W. T. Geng, Phys. Rev. B: Condens. Matter Mater. Phys., 2015, 91, 045433.

165 A. Lopez-Bezanilla, Phys. Rev. B: Condens. Matter Mater. Phys., 2016, 93, 035433.

166 F. Ersan, E. Aktu"rk and S. Ciraci, J. Phys. Chem. C, 2016, 120, 14345-14355.

167 Z. Li, W. Xu, Y. Yu, H. Du, K. Zhen, J. Wang, L. Luo, H. Qiu and X. Yang, J. Mater. Chem. C, 2016, 4, 362-370.

168 J. Du, C. Xia, Y. An, T. Wang and Y. Jia, J. Mater. Sci., 2016, 51, 9504-9513.

169 M. Bai, W. X. Zhang and C. He, J. Solid State Chem., 2017, 251, 1-6.

170 Y. Li, C. Xia, T. Wang, X. Tan, X. Zhao and S. Wei, Solid State Commun., 2016, 230, 6-10.

171 M. Liu, Q. Chen, Y. Huang, C. Cao and Y. He, Superlattices Microstruct., 2016, 100, 131-141. 
172 M. Sun, S. Wang, Y. Du, J. Yu and W. Tang, Appl. Surf. Sci., 2016, 389, 594-600.

173 C. Liu, C. Liu and X. Yan, Phys. Lett. A, 2017, 381, 1092-1096.

174 L. F. Yang, Y. Song, W. B. Mi and X. C. Wang, Appl. Phys. Lett., 2016, 109, 022103.

175 M. Xie, S. Zhang, B. Cai, Y. Zou and H. Zeng, RSC Adv., 2016, 6, 14620-14625.

176 G. Wang, R. Pandey and S. P. Karna, Nanoscale, 2015, 7, 524-531.

177 L. Zhu, S. S. Wang, S. Guan, Y. Liu, T. Zhang, G. Chen and S. A. Yang, Nano Lett., 2016, 16, 6548-6554.

178 D. W. Boukhvalov, A. N. Rudenko, D. A. Prishchenko, V. G. Mazurenko and M. I. Katsnelson, Phys. Chem. Chem. Phys., 2015, 17, 15209-15217.

179 A. Ziletti, A. Carvalho, P. E. Trevisanutto, D. K. Campbell, D. F. Coker and A. H. Castro Neto, Phys. Rev. B: Condens. Matter Mater. Phys., 2015, 91, 085407.

180 J. Dai and X. C. Zeng, RSC Adv., 2014, 4, 48017-48021.

181 Y. Wang, W. Ji, C. Zhang, P. Li, S. Zhang, P. Wang, S. Li and S. Yan, Appl. Phys. Lett., 2017, 110, 213101.

182 T. Zhou, J. Zhang, Y. Xue, B. Zhao, H. Zhang, H. Jiang and Z. Yang, Phys. Rev. B: Condens. Matter Mater. Phys., 2016 , $94,235449$.

183 T. Zhou, J. Zhang, B. Zhao, H. Zhang and Z. Yang, Nano Lett., 2015, 15, 5149-5155.

184 W. Zhou, S. Guo, X. Liu, B. Cai, X. Song, Z. Zhu and S. Zhang, Appl. Surf. Sci., 2018, 427, 363-368.

185 Z. Song, C. Liu, J. Yang, J. Han, M. Ye, B. Fu, Y. Yang, Q. Niu, J. Lu and Y. Yao, NPG Asia Mater., 2014, 6, e147.

186 Y. Ma, Y. Dai, L. Kou, T. Frauenheim and T. Heine, Nano Lett., 2015, 15, 1083-1089.

187 C. Liu, J. Zhou and Y. Yao, Phys. Rev. B: Condens. Matter Mater. Phys., 2015, 91, 165430.

188 C. Liu, S. Guan, Z. Song, S. A. Yang, J. Yang and Y. Yao, Phys. Rev. B: Condens. Matter Mater. Phys., 2014, 90, 085431.

189 C. Niu, G. Bihlmayer, H. Zhang, D. Wortmann, S. Blu“gel and Y. Mokrousov, Phys. Rev. B: Condens. Matter Mater. Phys., 2015, 91, 041303.

190 A. Avsar, I. J. Vera-Marun, J. Y. Tan, K. Watanabe, T. Taniguchi, A. H. Castro Neto and B. Ozyilmaz, ACS Nano, 2015, 9, 4138-4145.

191 L. E. Marsoner Steinkasserer, S. Suhr and B. Paulus, Phys. Rev. B: Condens. Matter Mater. Phys., 2016, 94, 125444.

192 Y. Deng, Z. Luo, N. J. Conrad, H. Liu, Y. Gong, S. Najmaei, P. M. Ajayan, J. Lou, X. Xu and P. D. Ye, ACS Nano, 2014, 8, 8292-8299.

193 K. Gong, L. Zhang, W. Ji and H. Guo, Phys. Rev. B: Condens. Matter Mater. Phys., 2014, 90, 125441.

194 X. Wang, M. Zebarjadi and K. Esfarjani, Nanoscale, 2016, 8, 14695-14704.

195 N. Gillgren, D. Wickramaratne, Y. Shi, T. Espiritu, J. Yang, J. Hu, J. Wei, X. Liu, Z. Mao, K. Watanabe, T. Taniguchi, M. Bockrath, Y. Barlas, R. K. Lake and C. Ning Lau, 2D Mater., 2014, 2, 011001.

196 L. Viti, J. Hu, D. Coquillat, A. Politano, C. Consejo, W. Knap and M. S. Vitiello, Adv. Mater., 2016, 28, 7390-7396.

197 X. Chen, Y. Wu, Z. Wu, Y. Han, S. Xu, L. Wang, W. Ye, T. Han, Y. He, Y. Cai and N. Wang, Nat. Commun., 2015, 6, 7315.

198 G. C. Constantinescu and N. D. Hine, Nano Lett., 2016, 16, 2586-2594.

199 H. Guo, N. Lu, J. Dai, X. Wu and X. C. Zeng, J. Phys. Chem. C, 2014, 118, 14051-14059.

200 J. Shim, S. Oh, D. H. Kang, S. H. Jo, M. H. Ali, W. Y. Choi, K. Heo, J. Jeon, S. Lee, M. Kim, Y. J. Song and J. H. Park, Nat. Commun., 2016, 7, 13413.

201 J. Yuan, S. Najmaei, Z. Zhang, J. Zhang, S. Lei, P. M. Ajayan, B. I. Yakobson and J. Lou, ACS Nano, 2015, 9, 555563.

202 Y. Cao, A. Mishchenko, G. L. Yu, E. Khestanova, A. P. Rooney, E. Prestat, A. V. Kretinin, P. Blake, M. B. Shalom, C. Woods, J. Chapman, G. Balakrishnan, I. V. Grigorieva, K. S. Novoselov, B. A. Piot, M. Potemski, K. Watanabe, T. Taniguchi, S. J. Haigh, A. K. Geim and R. V. Gorbachev, Nano Lett., 2015, 15, 4914-4921.

203 P. Rivero, C. M. Horvath, Z. Zhu, J. Guan, D. Tománek and S. Barraza-Lopez, Phys. Rev. B: Condens. Matter Mater. Phys., 2015, 91, 115413.

204 R. A. Doganov, E. C. O’Farrell, S. P. Koenig, Y. Yeo, A. Ziletti, A. Carvalho, D. K. Campbell, D. F. Coker, K. Watanabe, T. Taniguchi, A. H. Castro Neto and B. Ozyilmaz, Nat. Commun., 2015, 6, 6647.

205 W. Yu, Z. Zhu, S. Zhang, X. Cai, X. Wang, C. Y. Niu and

W. B. Zhang, Appl. Phys. Lett., 2016, 109, 103104.

206 J. E. Padilha, A. Fazzio and A. J. da Silva, Phys. Rev. Lett., 2015, 114, 066803.

207 H. Lu, J. Gao, Z. Hu and X. Shao, RSC Adv., 2016, 6, 102724.

208 Y. Song, D. Li, W. Mi, X. Wang and Y. Cheng, J. Phys. Chem. C, 2016, 120, 5613-5618.

209 Y. Wang and Y. Ding, Phys. Chem. Chem. Phys., 2015, 17, 27769-27776. 
210 C. Xia, B. Xue, T. Wang, Y. Peng and Y. Jia, Appl. Phys. Lett., 2015, 107, 193107.

211 H. Shu, Y. Tong and J. Guo, Phys. Chem. Chem. Phys., 2017, 19, 10644-10650.

212 X. Chen, Q. Yang, R. Meng, J. Jiang, Q. Liang, C. Tan and X. Sun, J. Mater. Chem. C, 2016, 4, 5434-5441.

213 S. Cui, H. Pu, S. A. Wells, Z. Wen, S. Mao, J. Chang, M. C. Hersam and J. Chen, Nat. Commun., 2015, 6, 8632.

214 V. Tayari, N. Hemsworth, I. Fakih, A. Favron, E. Gaufres, G. Gervais, R. Martel and T. Szkopek, Nat. Commun., $2015,6,7702$.

215 D. Li, H. Jussila, L. Karvonen, G. Ye, H. Lipsanen, X. Chen and Z. Sun, Sci. Rep., 2015, 5, 15899.

216 A. Favron, E. Gaufres, F. Fossard, A. L. Phaneuf-L'Heureux, N. Y. Tang, P. L. Levesque, A. Loiseau, R. Leonelli, S. Francoeur and R. Martel, Nat. Mater., 2015, 14, 826-832.

217 A. Castellanos-Gomez, L. Vicarelli, E. Prada, J. O. Island, K. L. Narasimha-Acharya, S. I. Blanter, D. J. Groenendijk, M. Buscema, G. A. Steele, J. V. Alvarez, H. W. Zandbergen, J. J. Palacios and G. S. J. van der Zant, 2D Mater., 2014, 1, 025001.

218 J. O. Island, G. A. Steele, H. S. J. van der Zant and

A. Castellanos-Gomez, 2D Mater., 2015, 2, 011002.

219 J. Yang, R. Xu, J. Pei, Y. W. Myint, F. Wang, Z. Wang,S. Zhang, Z. Yu and Y. Lu, Light: Sci. Appl., 2015, 4, e312.

220 S. Zhang, J. Yang, R. Xu, F. Wang, W. Li, M. Ghufran, Y.-W. Zhang, Z. Yu, G. Zhang and Q. Qin, ACS Nano, 2014, 8, 9590-9596.

221 R. Xu, S. Zhang, F. Wang, J. Yang, Z. Wang, J. Pei, Y. W. Myint, B. Xing, Z. Yu, L. Fu, Q. Qin and Y. Lu, ACS Nano, 2016, 10, 2046-2053.

222 J. D. Wood, S. A. Wells, D. Jariwala, K. S. Chen, E. Cho, V. K. Sangwan, X. Liu, L. J. Lauhon, T. J. Marks and M. C. Hersam, Nano Lett., 2014, 14, 6964-6970.

223 S. Ge, C. Li, Z. Zhang, C. Zhang, Y. Zhang, J. Qiu, Q. Wang, J. Liu, S. Jia and J. Feng, Nano Lett., 2015, 15, 46504656.

224 J. S. Kim, P. J. Jeon, J. Lee, K. Choi, H. S. Lee, Y. Cho, Y. T. Lee, D. K. Hwang and S. Im, Nano Lett., 2015, 15, $5778-5783$.

225 Y. Anugrah, M. C. Robbins, P. A. Crowell and S. J. Koester, Appl. Phys. Lett., 2015, 106, 103108.

226 Z. Luo, J. Maassen, Y. Deng, Y. Du, R. P. Garrelts, M. S. Lundstrom, P. D. Ye and X. Xu, Nat. Commun., 2015, 6, 8572.

227 Y. Saito and Y. Iwasa, ACS Nano, 2015, 9, 3192-3198.

228 J. R. Brent, N. Savjani, E. A. Lewis, S. J. Haigh, D. J. Lewis and P. O’Brien, Chem. Commun., 2014, 50, 1333813341.

229 Z. Guo, H. Zhang, S. Lu, Z. Wang, S. Tang, J. Shao, Z. Sun, H. Xie, H. Wang, X.-F. Yu and P. K. Chu, Adv. Funct. Mater., 2015, 25, 6996-7002.

230 P. Yasaei, B. Kumar, T. Foroozan, C. Wang, M. Asadi, D. Tuschel, J. E. Indacochea, R. F. Klie and A. SalehiKhojin, Adv. Mater., 2015, 27, 1887-1892.

231 D. Hanlon, C. Backes, E. Doherty, C. S. Cucinotta, N. C. Berner, C. Boland, K. Lee, A. Harvey, P. Lynch, Z. Gholamvand, S. Zhang, K. Wang, G. Moynihan, A. Pokle, Q. M. Ramasse, N. McEvoy, W. J. Blau, J. Wang, G. Abellan, F. Hauke, A. Hirsch, S. Sanvito, D. D. O’Regan, G. S. Duesberg, V. Nicolosi and J. N. Coleman, Nat. Commun., 2015, 6, 8563.

232 J. Kang, J. D. Wood, S. A. Wells, J. H. Lee, X. Liu, K. S. Chen and M. C. Hersam, ACS Nano, 2015, 9, 3596-3604.

233 W. Zhao, Z. Xue, J. Wang, J. Jiang, X. Zhao and T. Mu, ACS Appl. Mater. Interfaces, 2015, 7, 27608-27612.

234 J. Kang, S. A. Wells, J. D. Wood, J. H. Lee, X. Liu, C. R. Ryder, J. Zhu, J. R. Guest, C. A. Husko and M. C. Hersam, Proc. Natl. Acad. Sci. U. S. A., 2016, 113, 11688-11693.

235 H. Wang, X. Yang, W. Shao, S. Chen, J. Xie, X. Zhang, J. Wang and Y. Xie, J. Am. Chem. Soc., 2015, 137, 1137611382.

236 F. Xu, B. Ge, J. Chen, A. Nathan, L. L. Xin, H. Ma, H. Min,

C. Zhu, W. Xia, Z. Li, S. Li, K. Yu, L. Wu, Y. Cui, L. Sun and

Y. Zhu, 2D Mater., 2016, 3, 025005.

237 A. H. Woomer, T. W. Farnsworth, J. Hu, R. A. Wells, C. L. Donley and S. C. Warren, ACS Nano, 2015, 9, 88698884.

238 W. Lu, H. Nan, J. Hong, Y. Chen, C. Zhu, Z. Liang, X. Ma,

Z. Ni, C. Jin and Z. Zhang, Nano Res., 2014, 7, 853-859.

239 J. Jia, S. K. Jang, S. Lai, J. Xu, Y. J. Choi, J.-H. Park and S. Lee, ACS Nano, 2015, 9, 8729-8736.

240 Z. Yang, J. Hao, S. Yuan, S. Lin, H. M. Yau, J. Dai and P. P. Lau, Adv. Mater., 2015, 27, 3748-3754. 
241 A. Ambrosi, Z. Sofer and M. Pumera, Angew. Chem., 2017, 129, 10579-10581.

242 J. B. Smith, D. Hagaman and H. F. Ji, Nanotechnology, 2016, 27, 215602.

243 J. Gu, Z. Du, C. Zhang, J. Ma, B. Li and S. Yang, Adv. Energy Mater., 2017, 7, 1700447.

244 Y. Song, Z. Liang, X. Jiang, Y. Chen, Z. Li, L. Lu, Y. Ge, K. Wang, J. Zheng, S. Lu, J. Ji and H. Zhang, 2D Mater., $2017,4,045010$

245 M. Fortin-Deschenes, O. Waller, T. O. Mentes, A. Locatelli, S. Mukherjee, F. Genuzio, P. L. Levesque, A. Hebert, R. Martel and O. Moutanabbir, Nano Lett., 2017, 17, 4970-4975.

246 T. Nagao, T. Doi, T. Sekiguchi and S. Hasegawa, Jpn. J. Appl. Phys., 2000, 39, 4567.

247 P. Kumar, J. Singh and A. C. Pandey, RSC Adv., 2013, 3, 2313-2317.

248 M. Kammler and M. Horn-von Hoegen, Surf. Sci., 2005, 576, 56-60. 249 T. Nagao, S. Yaginuma, M. Saito, T. Kogure, J. T. Sadowski, T. Ohno, S. Hasegawa and T. Sakurai, Surf. Sci., 2005, 590, 247-252.

250 S. Yaginuma, T. Nagao, J. T. Sadowski, M. Saito, K. Nagaoka, Y. Fujikawa, T. Sakurai and T. Nakayama, Surf. Sci., 2007, 601, 3593-3600.

251 H. H. Sun, M. X. Wang, F. Zhu, G. Y. Wang, H. Y. Ma, Z. A. Xu, Q. Liao, Y. Lu, C. L. Gao, Y. Y. Li, C. Liu, D. Qian, D. Guan and J. F. Jia, Nano Lett., 2017, 17, 3035-3039.

252 Y. Lu, W. Xu, M. Zeng, G. Yao, L. Shen, M. Yang, Z. Luo, F. Pan, K. Wu, T. Das, P. He, J. Jiang, J. Martin, Y. P. Feng, H. Lin and X. S. Wang, Nano Lett., 2015, 15, 80-87.

253 E. S. Walker, S. R. Na, D. Jung, S. D. March, J. S. Kim, T. Trivedi, W. Li, L. Tao, M. L. Lee, K. M. Liechti, D. Akinwande and S. R. Bank, Nano Lett., 2016, 16, 6931-6938.

254 M. A. Meitl, Z. Zhu, V. Kumar, K. J. Lee, X. Feng, Y. Y. Huang, I. Adesida, R. G. Nuzzo and J. A. Rogers, Nat. Mater., 2005, 5, 33-38.

255 A. Castellanos-Gomez, M. Buscema, R. Molenaar, V. Singh, L. Janssen, H. S. J. van der Zant and G. A. Steele, 2D Mater., 2014, 1, 011002.

256 Y. Liu, H. Nan, X. Wu, W. Pan, W. Wang, J. Bai, W. Zhao, L. Sun, X. Wang and Z. Ni, ACS Nano, 2013, 7, 42024209.

257 A. Castellanos-Gomez, M. Barkelid, A. M. Goossens, V. E. Calado, H. S. van der Zant and G. A. Steele, Nano Lett., 2012, 12, 3187-3192.

258 H. S. Tsai, C. C. Lai, H. Medina, S. M. Lin, Y. C. Shih, Y. Z. Chen, J. H. Liang and Y. L. Chueh, Nanoscale, 2014, 6, $13861-13869$.

259 H. S. Tsai, Y. Z. Chen, H. Medina, T. Y. Su, T. S. Chou, Y. H. Chen, Y. L. Chueh and J. H. Liang, Phys. Chem. Chem. Phys., 2015, 17, 21389-21393.

260 H. S. Tsai, C. C. Lai, C. H. Hsiao, H. Medina, T. Y. Su, H. Ouyang, T. H. Chen, J. H. Liang and Y. L. Chueh, ACS Appl. Mater. Interfaces, 2015, 7, 13723-13727.

261 H. S. Tsai, C. H. Hsiao, C. W. Chen, H. Ouyang and J. H. Liang, Nanoscale, 2016, 8, 9488-9492.

262 M. Dobbelin, A. Ciesielski, S. Haar, S. Osella, M. Bruna,

A. Minoia, L. Grisanti, T. Mosciatti, F. Richard, E. A. Prasetyanto, L. De Cola, V. Palermo, R. Mazzaro, V. Morandi, R. Lazzaroni, A. C. Ferrari, D. Beljonne and P. Samori, Nat. Commun., 2016, 7, 11090.

263 L. Cai, J. He, Q. Liu, T. Yao, L. Chen, W. Yan, F. Hu, Y. Jiang, Y. Zhao, T. Hu, Z. Sun and S. Wei, J. Am. Chem. Soc., 2015, 137, 2622-2627.

264 J. Xie, H. Zhang, S. Li, R. Wang, X. Sun, M. Zhou, J. Zhou, X. W. Lou and Y. Xie, Adv. Mater., 2013, 25, 58075813.

265 J. Xie, J. Zhang, S. Li, F. Grote, X. Zhang, H. Zhang, R. Wang, Y. Lei, B. Pan and Y. Xie, J. Am. Chem. Soc., 2013, 135, 17881-17888.

266 H. Rau and A. Rabenau, J. Cryst. Growth, 1968, 3, 417-421.

267 Y. Xu, Z. Wang, Z. Guo, H. Huang, Q. Xiao, H. Zhang and X.-F. Yu, Adv. Opt. Mater., 2016, 4, 1223-1229.

268 H. Hu, B. Yang, Q. Li, X. Liu, W. Yu and Y. Qian, J. Cryst. Growth, 2004, 261, 485-489.

269 M. Zhang, Z. Wang, G. Xi, D. Ma, R. Zhang and Y. Qian, J. Cryst. Growth, 2004, 268, 215-221.

270 W. Debao, Y. Dabin, P. Yiyia, M. Zhaoyu, Z. Shuyuan and Q. Yitai, Nanotechnology, 2003, 14, 748-751.

271 J. Wang, X. Wang, Q. Peng and Y. Li, Inorg. Chem., 2004, 43, 7552-7556.

272 J. Wang and Y. Li, Adv. Mater., 2003, 15, 445-447.

273 F. Qin, G. Li, H. Xiao, Z. Lu, H. Sun and R. Chen, Dalton Trans., 2012, 41, 11263-11266.

274 J. N. Israelachvili, Intermolecular and surface forces, Academic Press, 2011.

275 K. S. Novoselov, A. K. Geim, S. V. Morozov, D. Jiang, Y. Zhang, S. V. Dubonos, I. V. Grigorieva and A. A. Firsov, Science, 2004, 306, 666-669. 
276 K. R. Paton, E. Varrla, C. Backes, R. J. Smith, U. Khan, A. O’Neill, C. Boland, M. Lotya, O. M. Istrate, P. King, T. Higgins, S. Barwich, P. May, P. Puczkarski, I. Ahmed, M. Moebius, H. Pettersson, E. Long, J. Coelho, S. E. O'Brien, E. K. McGuire, B. M. Sanchez, G. S. Duesberg, N. McEvoy, T. J. Pennycook, C. Downing, A. Crossley, V. Nicolosi and J. N. Coleman, Nat. Mater., 2014, 13, 624-630.

277 P. Nemes-Incze, Z. Osva'th, K. Kamara’s and L. P. Biro', Carbon, 2008, 46, 1435-1442.

278 X. Wang, K. Kunc, I. Loa, U. Schwarz and K. Syassen, Phys. Rev. B: Condens. Matter Mater. Phys., 2006, 74, 134305.

279 C. Y. Su, A. Y. Lu, Y. Xu, F. R. Chen, A. N. Khlobystov and L. J. Li, ACS Nano, 2011, 5, 2332-2339.

280 C. T. J. Low, F. C. Walsh, M. H. Chakrabarti, M. A. Hashim and M. A. Hussain, Carbon, 2013, 54, 1-21.

281 N. Liu, P. Kim, J. H. Kim, J. H. Ye, S. Kim and C. J. Lee, ACS Nano, 2014, 8, 6902-6910.

282 M. Shimizu, H. Usui, K. Yamane, T. Sakata, T. Nokami, T. Itoh and H. Sakaguchi, Int. J. Electrochem. Sci., 2015, 10, 10132-10144.

283 P. Yu, S. E. Lowe, G. P. Simon and Y. L. Zhong, Curr. Opin. Colloid Interface Sci., 2015, 20, 329-338.

284 F. Yang, K. Liu, K. Hong, D. Reich, P. Searson and C. Chien, Science, 1999, 284, 1335-1337.

285 Y. M. Koroteev, G. Bihlmayer, J. E. Gayone, E. V. Chulkov, S. Blugel, P. M. Echenique and P. Hofmann, Phys. Rev. Lett., 2004, 93, 046403.

286 T. Nagao, J. T. Sadowski, M. Saito, S. Yaginuma, Y. Fujikawa, T. Kogure, T. Ohno, Y. Hasegawa, S. Hasegawa and T. Sakurai, Phys. Rev. Lett., 2004, 93, 105501.

287 J. T. Sun, H. Huang, S. L. Wong, H. J. Gao, Y. P. Feng and A. T. Wee, Phys. Rev. Lett., 2012, 109, 246804.

288 Y. F. Ogrin, V. Lutskii and M. Elinson, Sov. J. Exp. Theor. Phys. Lett., 1966, 3, 71.

289 L. Kai-Tak, D. Zhipeng and G. Jing, IEEE Electron Device Lett., 2014, 35, 963-965.

290 L. Li, M. Engel, D. B. Farmer, S. J. Han and H. S. Wong, ACS Nano, 2016, 10, 4672-4677.

291 H. Wang, X. Wang, F. Xia, L. Wang, H. Jiang, Q. Xia, M. L. Chin, M. Dubey and S. J. Han, Nano Lett., 2014, 14, 6424-6429.

292 L. Viti, J. Hu, D. Coquillat, A. Politano, W. Knap and M. S. Vitiello, Sci. Rep., 2016, 6, 20474.

293 R. Wan, X. Cao and J. Guo, Appl. Phys. Lett., 2014, 105, 163511.

294 L. Fei, W. Yijiao, L. Xiaoyan, W. Jian and G. Hong, IEEE Trans. Electron Devices, 2014, 61, 3871-3876.

295 C. Xi and G. Jing, IEEE Trans. Electron Devices, 2015, 62, 659-665.

296 C. Wang, R. Cheng, L. Liao and X. Duan, Nano Today, 2013, 8, 514-530.

297 Y. Du, H. Liu, Y. Deng and P. D. Ye, ACS Nano, 2014, 8, 10035-10042.

298 S. P. Koenig, R. A. Doganov, H. Schmidt, A. Castro Neto and B. Ozyilmaz, Appl. Phys. Lett., 2014, 104, 103106.

299 W. Zhu, M. N. Yogeesh, S. Yang, S. H. Aldave, J. S. Kim, S. Sonde, L. Tao, N. Lu and D. Akinwande, Nano Lett., 2015, 15, 1883-1890.

300 H. Liu, A. T. Neal, M. Si, Y. Du and D. Y. Peide, IEEE Trans. Electron Devices, 2014, 35, 795-797.

301 N. Haratipour, S. Namgung, S. H. Oh and S. J. Koester, ACS Nano, 2016, 10, 3791-3800.

302 D. Xiang, C. Han, J. Wu, S. Zhong, Y. Liu, J. Lin, X. A. Zhang, W. Ping Hu, B. Ozyilmaz, A. H. Neto, A. T. Wee and W. Chen, Nat. Commun., 2015, 6, 6485.

303 N. Youngblood, C. Chen, S. J. Koester and M. Li, Nat. Photonics, 2015, 9, 247-252.

304 S. Das, W. Zhang, M. Demarteau, A. Ho $\square$ mann, M. Dubey and A. Roelofs, Nano Lett., 2014, 14, 5733-5739.

305 M. V. Kamalakar, B. N. Madhushankar, A. Dankert and S. P. Dash, Small, 2015, 11, 2209-2216.

306 J. S. Kim, Y. Liu, W. Zhu, S. Kim, D. Wu, L. Tao, A. Dodabalapur, K. Lai and D. Akinwande, Sci. Rep., $2015,5,8989$.

307 J. Na, Y. T. Lee, J. A. Lim, D. K. Hwang, G. T. Kim, W. K. Choi and Y. W. Song, ACS Nano, 2014, 8, 1175311762.

308 X. Luo, Y. Rahbarihagh, J. C. M. Hwang, H. Liu, Y. Du and P. D. Ye, IEEE Electron Device Lett., 2014, 35, 13141316.

309 S. B. Lu, L. L. Miao, Z. N. Guo, X. Qi, C. J. Zhao, H. Zhang, S. C. Wen, D. Y. Tang and D. Y. Fan, Opt. Express, 2015, 23, 11183-11194.

310 T. Takahashi, K. Shirotani, S. Suzuki and T. Sagawa, Solid State Commun., 1983, 45, 945-948.

311 L. Li, F. Yang, G. J. Ye, Z. Zhang, Z. Zhu, W. Lou, X. Zhou, L. Li, K. Watanabe, T. Taniguchi, K. Chang, Y. Wang, X. H. Chen and Y. Zhang, Nat. Nanotechnol., 2016, 11, 593-597.

312 H. J. Conley, B. Wang, J. I. Ziegler, R. F. Haglund, Jr., S. T. Pantelides and K. I. Bolotin, Nano Lett., 2013, 13, 3626-3630.

313 S. Yuan, A. N. Rudenko and M. I. Katsnelson, Phys. Rev. B: Condens. Matter Mater. Phys., 2015, 91, 115436.

314 G. Zhang, S. Huang, A. Chaves, C. Song, V. O. Ozcelik, T. Low and H. Yan, Nat. Commun., 2017, 8, 14071. 
315 J. Wu, G. K. W. Koon, D. Xiang, C. Han, C. T. Toh, E. S. Kulkarni, I. Verzhbitskiy, A. Carvalho, A. S. Rodin and S. P. Koenig, ACS Nano, 2015, 9, 8070-8077.

316 M. Buscema, D. J. Groenendijk, S. I. Blanter, G. A. Steele, H. S. van der Zant and A. Castellanos-Gomez, Nano Lett., 2014, 14, 3347-3352.

317 T. Low, M. Engel, M. Steiner and P. Avouris, Phys. Rev. B: Condens. Matter Mater. Phys., 2014, 90, 081408.

318 M. Engel, M. Steiner and P. Avouris, Nano Lett., 2014, 14, 6414-6417.

319 H. Yuan, X. Liu, F. Afshinmanesh, W. Li, G. Xu, J. Sun, B. Lian, A. G. Curto, G. Ye, Y. Hikita, Z. Shen, S. C. Zhang, X. Chen, M. Brongersma, H. Y. Hwang and Y. Cui, Nat. Nanotechnol., 2015, 10, 707-713.

320 M. Buscema, D. J. Groenendijk, G. A. Steele, H. S. van der Zant and A. Castellanos-Gomez, Nat. Commun., 2014, 5, 4651.

321 X. Yu, S. Zhang, H. Zeng and Q. J. Wang, Nano Energy, 2016, 25, 34-41.

322 L. Viti, J. Hu, D. Coquillat, W. Knap, A. Tredicucci, A. Politano and M. S. Vitiello, Adv. Mater., 2015, 27, 55675572 .

323 J. Buckley, B. De Salvo, D. Deleruyelle, M. Gely, G. Nicotra, S. Lombardo, J. F. Damlencourt, P. Hollinger, F. Martin and S. Deleonibus, Microelectron. Eng., 2005, 80, 210-213.

324 C. R. Ryder, J. D. Wood, S. A. Wells, Y. Yang, D. Jariwala, T. J. Marks, G. C. Schatz and M. C. Hersam, Nat. Chem., 2016, 8, 597-602.

325 J. Kim, S. S. Baik, S. H. Ryu, Y. Sohn, S. Park, B.-G. Park, J. Denlinger, Y. Yi, H. J. Choi and K. S. Kim, Science, 2015, 349, 723-726.

326 L. Li, J. Kim, C. Jin, G. J. Ye, D. Y. Qiu, F. H. da Jornada, Z. Shi, L. Chen, Z. Zhang, F. Yang, K. Watanabe, T. Taniguchi, W. Ren, S. G. Louie, X. H. Chen, Y. Zhang and F. Wang, Nat. Nanotechnol., 2017, 12, 21-25.

327 S. Ge, L. Zhang, P. Wang and Y. Fang, Sci. Rep., 2016, 6, 27307.

328 S. Aghaeimeibodi, J. H. Kim and E. Waks, 2017, arXiv: 1706.10189.

329 C. Dutreix, E. A. Stepanov and M. I. Katsnelson, Phys. Rev. B: Condens. Matter Mater. Phys., 2016, 93, 241404.

330 J. J. Zhou, W. Feng, C. C. Liu, S. Guan and Y. Yao, Nano Lett., 2014, 14, 4767-4771.

331 A. N. Abbas, B. Liu, L. Chen, Y. Ma, S. Cong, N. Aroonyadet, M. Ko"pf, T. Nilges and C. Zhou, ACS Nano, 2015, 9, 5618-5624.

332 S. Y. Cho, Y. Lee, H. J. Koh, H. Jung, J. S. Kim, H. W. Yoo, J. Kim and H. T. Jung, Adv. Mater., 2016, 28, 70207028.

333 C. C. Mayorga-Martinez, Z. Sofer and M. Pumera, Angew. Chem., Int. Ed., 2015, 127, 14525-14528.

334 M. S. Khan, A. Srivastava and R. Pandey, RSC Adv., 2016, 6, 72634-72642.

335 C. M. Park, J. H. Kim, H. Kim and H. J. Sohn, Chem. Soc. Rev., 2010, 39, 3115-3141.

336 W. Li, Y. Yang, G. Zhang and Y. W. Zhang, Nano Lett., 2015, 15, 1691-1697.

337 D. Wang, G.-C. Guo, X.-L. Wei, L.-M. Liu and S. J. Zhao, J. Power Sources, 2016, 302, 215-222.

338 S. Zhao, W. Kang and J. Xue, J. Mater. Chem. A, 2014, 2, 19046-19052.

339 Q. Yao, C. Huang, Y. Yuan, Y. Liu, S. Liu, K. Deng and E. Kan, J. Phys. Chem. C, 2015, 119, 6923-6928.

340 Z.-S. Wu, W. Ren, L. Xu, F. Li and H.-M. Cheng, ACS Nano, 2011, 5, 5463-5471.

341 C. Uthaisar and V. Barone, Nano Lett., 2010, 10, 2838-2842.

342 Y. Li, D. Wu, Z. Zhou, C. R. Cabrera and Z. Chen, J. Phys. Chem. Lett., 2012, 3, 2221-2227.

343 K. Persson, Y. Hinuma, Y. S. Meng, A. Van der Ven and G. Ceder, Phys. Rev. B: Condens. Matter Mater. Phys., $2010,82,125416$

344 X. Liu, Y. Wen, Z. Chen, B. Shan and R. Chen, Phys. Chem. Chem. Phys., 2015, 17, 16398-16404.

345 V. V. Kulish, O. I. Malyi, C. Persson and P. Wu, Phys. Chem. Chem. Phys., 2015, 17, 13921-13928.

346 J. Sun, G. Zheng, H. W. Lee, N. Liu, H. Wang, H. Yao, W. Yang and Y. Cui, Nano Lett., 2014, 14, 4573-4580.

347 J. Sun, H. W. Lee, M. Pasta, H. Yuan, G. Zheng, Y. Sun, Y. Li and Y. Cui, Nat. Nanotechnol., 2015, 10, 980-985.

348 L. Chen, G. Zhou, Z. Liu, X. Ma, J. Chen, Z. Zhang, X. Ma, F. Li, H. M. Cheng and W. Ren, Adv. Mater., 2016, 28, $510-517$.

349 X. Zhang and L. Zhao, J. Materiomics, 2015, 1, 92-105.

350 L. Pan, H. J. Liu, X. J. Tan, H. Y. Lv, J. Shi, X. F. Tang and G. Zheng, Phys. Chem. Chem. Phys., 2012, 14, 1358813593.

351 A. J. Minnich, M. S. Dresselhaus, Z. F. Ren and G. Chen, Energy Environ. Sci., 2009, 2, 466-479.

352 P. Pichanusakorn and P. Bandaru, Mater. Sci. Eng., R, 2010, 67, 19-63.

353 S. Konabe and T. Yamamoto, Appl. Phys. Express, 2015, 8, 015202.

354 S. K. Gupta, Y. Sonvane, G. Wang and R. Pandey, Chem. Phys. Lett., 2015, 641, 169-172.

355 L. Medrano Sandonas, D. Teich, R. Gutierrez, T. Lorenz, A. Pecchia, G. Seifert and G. Cuniberti, J. Phys. Chem. C, 2016, 120, 18841-18849.

356 H. Y. Lv, W. J. Lu, D. F. Shao and Y. P. Sun, Phys. Rev. B: Condens. Matter Mater. Phys., 2014, 90, 085433.

357 J. W. Jiang, Nanotechnology, 2015, 26, 055701.

358 S. Lee, F. Yang, J. Suh, S. Yang, Y. Lee, G. Li, H. Sung Choe, A. Suslu, Y. Chen, C. Ko, J. Park, K. Liu, J. Li, K. Hippalgaonkar, J. J. Urban, S. Tongay and J. Wu, Nat. Commun., 2015, 6, 8573.

359 A. Jain and A. J. McGaughey, Sci. Rep., 2015, 5, 8501. 
360 W. Tao, X. Ji, X. Xu, M. A. Islam, Z. Li, S. Chen, P. E. Saw, H. Zhang, Z. Bharwani, Z. Guo, J. Shi and O. C. Farokhzad, Angew. Chem., Int. Ed., 2017, 56, 11896-11900.

361 M. Xie, S. Zhang, B. Cai, Y. Gu, X. Liu, E. Kan and H. Zeng, Nano Energy, 2017, 38, 561-568. 